\title{
ANALYSIS OF THE OPTICS OF THE FINAL FOCUS TEST BEAM USING LIE ALGEBRA BASED TECHNIQUES*
}

\author{
Ghislain J. Roy \\ Stanford Linear Accelerator Center \\ Stanford University \\ Stanford, California 94309
}

September 1992

Prepared for the Department of Energy

under contract number DE-AC03-76SF00515

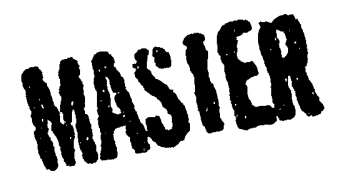

Printed in the United States of America. Available from the National Technical Information Service, U.S. Department of Commerce, 5285 Port Royal Road, Springfield, Virginia 22161.

*Ph. D. thesis 


\section{Table of Conterts}

1. The Optics of the Final Focus Test Beam . . . . . . . . . . . . . . . . 11

1.1. Introduction . . . . . . . . . . . . . . . . . . . . . . . . . . 11

1.2. Final focms systems . . . . . . . . . . . . . . . . . . . . . . . 11

1.2.1. Telescopes . . . . . . . . . . . . . . . . . . . . . . . . . 11

1.2.2. Chromaticity . . . . . . . . . . . . . . . . . . . . . . 12

1.2.3. Chromaticity correction . . . . . . . . . . . . . . . . . . . 13

1.3. FFTB optics . . . . . . . . . . . . . . . . . . . . . . . . . . 14

1.3.1. Optical modules . . . . . . . . . . . . . . . . . . . 15

1.3.2. Final Transformer . . . . . . . . . . . . . . . . . . . . . . 16

1.3.3. Chromatic Correction Sections . . . . . . . . . . . . . . . 18

1.3.4. Beta Exchanger . . . . . . . . . . . . . . . . . . . . . . . 19

1.3.5. Beta Matching Section . . . . . . . . . . . . . . . . . . . . 19

1.3.6. Dump line. . . . . . . . . . . . . . . . . . . . . . . . . 20

1.4. History of the design . . . . . . . . . . . . . . . . . . . . . . . 20

1.4.1. Early choices . . . . . . . . . . . . . . . . . . . . . . . 20

1.4.2. Evolution of the present solution . . . . . . . . . . . . . . . 21

1.j. Performances . . . . . . . . . . . . . . . . . . . . . . . . 22

1.j.1. A measure of final focus performance . . . . . . . . . . . . . 22

1.5.2. Residual aberrations - limits on the spot size . . . . . . . . . . 24

1.5.3. Adrlitional effects . . . . . . . . . . . . . . . . . . . . . . 27

1.5.4. Higher order inrestigation . . . . . . . . . . . . . . . . . . 28

2. Lie Algebra. . . . . . . . . . . . . . . . . . . . . . . . . . . . . 31

2.1. Introduction .......................... . . 31

2.2. Defintion . . . . . . . . . . . . . . . . . . . . 31

2.2.1. Algobra . . . . . . . . . . . . . . . . . . . . 31

2.2.2. Lir $A \lg (\operatorname{lol}$. . . . . . . . . . . . . . . . . . . . . . . . 32

2.2.3. Exampless . . . . . . . . . . . . . . . . . . . . . . 32

2.3. Hamiltonians and Poisson Brackets . . . . . . . . . . . . . . . 33

2.4. The Lie Algebras on Hamiltonians. . . . . . . . . . . . . . . . . 34

2.5. Lie (Operators . . . . . . . . . . . . . . . . . . . . . . . . . . 35

2.6. Lie Transformations and properties . . . . . . . . . . . . . . . . 36 
2.6.1. Definition . . . . . . . . . . . . . . . 36

2.6.2. Properties . . . . . . . . . . . . . . . . . . . 37

2.6.3. Examples . . . . . . . . . . . . . . . . . . . . 37

2.6.3.1. Uniform motion . . . . . . . . . . . . . . . . . 37

2.6.3.2. The harmonic oscillator. . . . . . . . . . . . . . . . . 40

2.7. Theorems . . . . . . . . . . . . . . . . . . . . . 41

2.8. Conclusion . . . . . . . . . . . . . . . . . . . . . . . 42

3. Application to Optics . . . . . . . . . . . . . . . . . . . . . . . . . 45

3.1. Introduction . . . . . . . . . . . . . . . . . . . . . . . 45

3.2. Hamiltonians . . . . . . . . . . . . . . . . . . . . . . . . . . 45

3.2.1. The Electro-Magnetic Hamiltonian . . . . . . . . . . . . . . 45

3.2.2. Approximations . . . . . . . . . . . . . . . . . . . . . . . 46

3.2.3. Potentials . . . . . . . . . . . . . . . . . . . . . . . 47

3.2.3.1. dipoles . . . . . . . . . . . . . . . . . . . . . . . . . 48

3.2.3.2. multipoles . . . . . . . . . . . . . . . . . . . . . . 48

3.2.3.3. summary . . . . . . . . . . . . . . . . . . . . . . 50 50

3.3. Principles . . . . . . . . . . . . . . . . . . . . . . . . . 50

3.3.1. Thin elements . . . . . . . . . . . . . . . . . . . . . . . 50

3.3.2. Linear Transformations . . . . . . . . . . . . . . . 51

3.3.3. Outline of the Methods . . . . . . . . . . . . . . . . . . . 51

3.4. Thick Elements . . . . . . . . . . . . . . . . . . . . . . . . . 52

3.4.1. Method . . . . . . . . . . . . . . . . . . . 52

3.4.2. First order in $\mathrm{CBH}$. . . . . . . . . . . . . . . . . . . . 53

3.4.2.1. multipoles . . . . . . . . . . . . . . . . . . . . . 53

3.4.2.2. quadrupoles . . . . . . . . . . . . . . . . . 53

3.4.3. second-order in $\mathrm{CBH}$. . . . . . . . . . . . . . . . . . . . 54

3.5. Beam Line Analysis . . . . . . . . . . . . . . . . . . . . . . . 57

3.5.1. First approach . . . . . . . . . . . . . . . . . 57

3.5.2. Looking for insight . . . . . . . . . . . . . . . . . . . . . 58

3.5.3. Geometrics cancellation in the CCS . . . . . . . . . . . . . . 59

3.5.4. Fifth order aberrations . . . . . . . . . . . . . . . 60

3.5.5. Other uses of this formalism . . . . . . . . . . . 63

3.6. Errors and offsets . . . . . . . . . . . . . . . . . . . . . . . 64 
3.7. Conclusion . . . . . . . . . . . . . . . . . . . . . . . . 65

4. Aberrations at the FilB . . . . . . . . . . . . . . . . . . . . . . . 6 i

4.1. Introduction . . . . . . . . . . . . . . . . . . . . . . . . . . 6 T

4.2. Classification . . . . . . . . . . . . . . . . . . . . . . . . . . 68

4.3. Number of aberrations . . . . . . . . . . . . . . . . . . . . . . 68

4.3.1. Symplecticity . . . . . . . . . . . . . . . . . . . . 68

4.3.2. Numbers. . . . . . . . . . . . . . . . . . . . . . . 69

4.3.3 Significant terms . . . . . . . . . . . . . . . . . . . . 70

4.4. Summary . . . . . . . . . . . . . . . . . . . . . . . . . . . . 71

4.5. First and Second Order . . . . . . . . . . . . . . . . . . . . . 72

4.6. Method of analysis . . . . . . . . . . . . . . . . . . . . . . 75

4.7. Third order . . . . . . . . . . . . . . . . . . . . . . . . . . . 77

4.8. Fourth order . . . . . . . . . . . . . . . . . . . . . . . . . . 79

4.9. Fifth order . . . . . . . . . . . . . . . . . . . . . . . . . . . 80

4.10. Higher order . . . . . . . . . . . . . . . . . . . . . . . . . . 80

4.11. Conclusion . . . . . . . . . . . . . . . . . . . . . . . . . . . 81

5. Stability Tolerances. . . . . . . . . . . . . . . . . . . . . . . . . . . 83

5.1. Tolerance Budget. . . . . . . . . . . . . . . . . . . . . . . . . 83

5.2. Steering. . . . . . . . . . . . . . . . . . . . 84

5.3. Dispersion. . . . . . . . . . . . . . . . . . . . . . . . . . . . 87

5.3.1. Final quadrupoles . . . . . . . . . . . . . . . . . . . . . . 89

5.3.2. Other quadrupoles . . . . . . . . . . . . . . . . . . . . . 90

5.3.3. Sextupoless. . . . . . . . . . . . . . . . . . . . . . . . . 91

5.t. Normal Quadrupole. . . . . . . . . . . . . . . . . . . . . . . . 91

5.j. Horizontal Sextupole Alignment. . . . . . . . . . . . . . . . . . 93

5.5.1. Sextupoles . . . . . . . . . . . . . . . . . . . . . . 93

5.5.2. Orbit offset . . . . . . . . . . . . . . . . . . . . . . . . 94

5.5.3. Bending magnets . . . . . . . . . . . . . . . . . . . . . . 95

5.6. Skew Quadrupole. . . . . . . . . . . . . . . . . . . . . . . . . 95

5.7. Vertical Sextmpole Aligmment. . . . . . . . . . . . . . . . . . . 98

5.7.1. Sextupoles . . . . . . . . . . . . . . . . . . . . . . . . 98

כ.T.2. Orbit offset . . . . . . . . . . . . . . . . . . . . . . . . 99

5.7.3. Bending mangnets . . . . . . . . . . . . . . . . . . . . . 99 
5.8 Sextupole and Skew Sextupole _. . . . . . . . . . . . . . . . . 100

5.9 Conclusion . . . . . . . . . . . . . . . . . . . . . . . . . . . 102

Conclusions . . . . . . . . . . . . . . . . . . . . . . . . 105

Appendix A . . . . . . . . . . . . . . . . . . . . . . . . . . . . . . . . . 109

Appendix B . . . . . . . . . . . . . . . . . . . . . . . . 121

References . . . . . . . . . . . . . . . . . . . . . . . . . . . 128 


\section{Acknowledgments}

I first want to thank Professor Jean Buon. He initiated my steps on the way to accelcrator physics. His teaching and friendship are very precious.

My thoughts also go to John Irwin. He also taught me a great deal. For the numerous discussions, your great patience and disponibility, thank you John.

To Michel Davier who accepted to chair the jury and to Jean-Eudes Augustin for being part of it, my sincere thanks. Olivier Napoly also accepted the position of "rapporteur" for this thesis and I thank him for this; his comments are always important to me.

I want to express my very sincere thanks to J.M. Paterson and Ron Ruth for hosting me in their Accelerator Theory and Special Projects group at SLAC for these three years. It has been a great privilege to learn among some of the greatest. Special thanks go to Matt Sands.

I am indebted to the FFTB Optics group. Thanks to K. Oide, K. Brown, R. Helm, N. Yamamoto, F. Bulos. Special thanks to Peter Tenenbaum and Rick Iverson for your questions and the discussions they triggered. Of course I want to express my gratitude to the entire FFTB collaboration. A special mention goes to Bernie Denton, Gerry Fischer, Finn Halbo and Dieter Walz; Their great experience and very high professionalism were remarkable. They have been true models for me. Thank you also Dave Burke for leading the FFTB with such enthusiasm.

For their help with the codes and machines, let me acknowledge the help and advice of M. Berz, A. Dragt, R. Servranckx and T. Kinight.

A special thought goes to those at SLAC who have always encouraged me and have become true friends. Nick Walker, Paul Emma, Tanya Boysen, Bill Gabella and the happy group. Let them be thanked here. A special word must go to Lia Merminga; For these discussions and advices late at night, thank you so much.

And to all who have shown their support; the friends from Stanford but also the members of the St Ann's choir, their families and friends.

To all of you, I dedicate this work. 


\section{Introduction}

High energy $e^{+} e^{--}$colliders are currently limited on the way to energies higher than $100 \mathrm{GeV}$ by synchrotron radiation. When their trajectories are bent by a magnetic field, charged particles emit electromagnetic radiation. The power radiated is proportional to the fourth power of the energy of the particles and inversely proportional to the square of the bending radius of the trajectory. In order to keep the radiated power down to a manageable level, the highest energy $e^{+} e^{-}$collider ring to date, LEP at CERN, has a circumference of about 27 kilometers for a maximum energy of $100 \mathrm{GeV}$ per beam.

One requirement for colliders in the next twenty years is to increase the (nlergy by an order of magnitude and reach the $1 \mathrm{TeV}$ center-of-mass energy for et $\mathrm{e}^{-}$collisions. The scaling of LEP to such energies, keeping a constant radiated power per unit length, would lead to a ring twenty five times larger or about 700 kilometers in circumference. Clearly not an economically feasible machine. The other way to build high-energy machines, which would not require such a gigantic size, is the linear collider. Here two linear accelerators facing each other accelerate the two beams. They are brought in collisions at the Interaction Point and discarded. The concept of such a machine has been tested at SLAC with the Stanford Linear Collider (SLC ${ }^{[(])}$) which has been a success as such; a number of specific: problems have been studied, some even discovered (muon shielding, collination) and for others the true complexity of these machines has been unveiled. This is particularly true for the problems linked with production and conservation of small emittances as well as for final focus systems. However the SLC is not a true linear collider. The beams are accelerated concurrently in a single linac then two arcs and final focus lines bring the beams in collision at the IP. The SLC has nonetheless demonstrated the feasibility of such linear machines. The lessons learned form the SLC could not have been obtained by any other way.

The other challenge of high-energy $e^{+} e^{-}$colliders is the luminosity required to investigate very rare events. The next generation of machines will require a luninosity between $10^{33}$ and $10^{34} \mathrm{~cm}^{-2} \mathrm{~s}^{-1}$. There are three main parameters to the luminosity: The frecuency of the collisions, the population of eacis bunch and the cross-sectional area of the beams at the IP. The first two parameters are the strong point of circular machines. The circulating current, 
proportional to the bunch populations times the repetition frecuency of the collisions. is fairly high albeit subject to limitations. For LEP the revolution frequency is of the order of $10^{4} \mathrm{H} z$ and a typical bunch population in colliding mode is $10^{12}$ particles corresponding to a current of the oider of a milliampere per bunch. On the other hand it is not possible to achieve very small beam sizes in circular machines since the beam-beam instabilities (disruption) could lead to the loss of the beams.

This last parameter is the strong point of linear colliders: Since the beams are discarded after the collision, it is possible to have very small spot sizes at the IP and lun in a disrupted mode where the beam-beam effects are very strong. On the other hand, the wakefields excited by the beams in the linacs, as well as the total "wall-plug" power used by the machine restrict the repetition frequency, of the order of $100 \mathrm{~Hz}$, and bunch population, $10^{11}$ particles per train of ten microbunches. Studies are actively carried out to solve these problems but the most promising way of achieving a high luminosity at a future linear collicler is to focus the beams very strongly at the IP.

This is the task of the Final Focus System, together with keeping the beans in collision. At the SLC the final focus delivers a round beam at the IP with transverse dimensions of the order of two microns. Typically a next generation linear collider requires flat beams a third of a micron wide and only a few nanometers high. The experience gained at the SLC in building and operating such a line is extremely valuable. However the new scale for spot sizes in linear collider projects prompts for more careful studies of such systems. The design of the optics calls for more stringent cancellation of stronger aberations. The tight requirements on tolerances and stability, both mechanical and electrical. the precision required for the beam instrumentation and more powerful techniques for diagnostics and correction of the beamline have to be investigated and tested.

The Final Focus Test Bean is being built at SLAC by an international collaboration of physicists and engineers from different laboratories worldwide: SLAC, KEK, INPNovosibirsk, LAL-Orsay, DESY and MPI-Munich participate in this enterprisc. Lsing the $50 \mathrm{GeV}$ electron beam from the SLAC linac, the goal is to form and measure a spot size $1 \mu \mathrm{m}$ wide and $60 \mathrm{~nm}$ high at the focal point of the system. Designed with possible parancters of a future linear collider in mind, the FFTB is a scaled prototype of most of the hardwaie, software and procedures required for the building and operation of a final focus system for a future linear collider. The demagnifications and the values of the betatron functions at the 
IP are identical to those for the Next Linear Collider (NLC). The emittances and the energy of the beam establish the scale of the FFTB compared to the NLC.

The optics of the FFTB is first presented in chapter 1. The principle of the correction of the chromatic aberrations of the final quadrupoles is described. The introduction of sextupoles and bending magnets for this cancellation gives rise to new non-linear chromatic aberrations. The FFTB layout, section by section, is then detailed and finally the most important remaining aberrations are discussed. The intent of this chapter is simply to introduce the reader to the FFTB, the layout, main features and the notations used in the rest of this thesis. More information can be found in the FFTB design report ${ }^{[3]}$ and the design handbook in preparation.

The motivation for the work presented in this thesis is the following. Since we are trying to achieve a very small spot size by careful cancellaticn of some strong aberrations, it is important not only to check that the aberration content of the lattice is low enough - this can be done with tracking codes - but most importantly to understand the mechanisms by which cancellations happen or new aberrations arise. Most mathematical tools for the study of theoretical optics go only to second order. They are based on the matrix formulation ${ }^{[6]}$ of Courant and Snyder that was made popular by the program ${ }^{[8]}$ Transport. A few good references can ba found in the bibliography.

The optics of the FFTB is corrected at second order. The investigation of third and higher order effects, which currently limit the performance of our designs, is crucial for the optimization of the design of the final focus systems for a next generation linear collider. The extension of the Transport formalism to third order has been started ${ }^{[11]}$ but this is not an easy tasi. Also it is not easy to find one's way in this wealth of coefficients. Although some higher order effects have been identified at the FFTB using an analytical approach based on kicks, there is no general way to systematically study these aberrations at third and higher order.

There exists a theory based on Differential Algebra which has been in use for some time, mostly applied to circular accelerators. Pioneered in this field by, among others, E. Forest and M. Berz, DA methods provide so called maps of a given system. A map is the representation of the transformation of a function and can be given at very high orders from the Hamiltonian of the system. DA techniques are currently being used mostly, instead of tracking, for stability studies for large hadron colliders such as the SSC in the United States 
and the LHC at CERN. It is indeed possible to concatenate the maps of indiviclual clements and form a high order map of one turn of a very large machine. Successive applications of this map has then been shown to give results equivalent to tracking results. However the concatenation of those high order maps does not provide very much insight on the problem and especially on the nechanism by which aberrations cancel or a new aberration arises from the interaction of lower order effects. And this is our primary concern in the design of final focus systems.

Another theory has been known for some time. Alex Dragt has proposed in the seventies to apply the theory of Lie transformations to some dynamical problems. ${ }^{[19,18]}$ Assuming one knows the Hamiltonian of a system the solution is simply and elegantly given by the associated Lie transformation applied to the functions associated with the canonical variables. Although this work was known it has not been much applied outside of the computer code Marylie $^{[13]}$ developed by Dragt and his team. One reason for this is, I believe, the decomposition chosen in order to separate the effects of different orders in a product of independent. Lie transformations. This stems directly from the Factorization Theorem ${ }^{[19]}$ of Dragt and Finn. Even for simple elements it is sometimes non-trivial to find this decomposition at order higher than three; fifth order contributions for most elements are now being incorporated to Marylie.

A simpler formul:ation was then proposed ${ }^{[20]}$ by John Irwin and used for the first time at the FFTB for the analysis of the optics. The expansion used is simpler since it consists only in the separation of linear and nonlinear terms. The design linear terms are then troated using the matrix formalism while the non-linear terms are treated separately. The concatenation is performed using the $\mathrm{CBH}$ theorem. The simplification arises mostly from the fact that the arguments of the Lie transformations are mostly the potentials of the elements to be considered, which are easily accessible.

Chapter 2 exposes the mathematical basis needed „o establish the physics in chapter 3. The theory of Lie groups and Lie algebra is generally non trivial. However the subset needed for the application to optics is simple. The notions of Lin algebra and operators on these algebras are reviewed. Then the central definition of the Lie transformation is present.ed. The simple examples of the uniform motion and the harmonic osciilator are cletailed, using both the resolution of the equations of motion derived from Hamilton's equations, and the application of the Lie transformation. These two examples are reyg similar to the arse of 
the motion of a charged particle in a drift space and a quadrupole respectively. Finally the theorem of the similarity transformation and the Campbell-Baker-Hausdorff theorem are presented. They are the two essential tools on which the methods presented in this thesis stand.

The application of these mathematical concepts to the physics of magnetic optics is the subject of chapter 3. I start here from the general electromagnetic Hamiltonian of a charged particle in a magnetic prtential typical of accelerator magnets. Some assumptions are made at this point that enable us to simplify the analytical calculations. It should be emphasized that these restrictions are not essential to the method and should the need arise to remove one or more of these assumptions, the same type of analysis would still be possible, albeit with a heavier formalism. The potentials used are expressed for dipoles and multipoles. The Lie transformation associated to the Hamiltonians with these potentials, when applied to the angular position functions of the particle, gives the kick, or change in direction, applied to the particle. This is essentially the expression in the thin lens approximation. The long element expression is then built using the $\mathrm{CBH}$ theorem and linear changes of coordinates. The same method lets us build a beamline composed of different long elements. The method is described in detail and involves the separation of the design linear optics from the non-linear and error-induced effects.

Finally the last part of this third chapter shows how more elaborate manipulations can be done using the methods presented. The example of the Chromatic Correction Section is taken. The well known cancellation of the geometric aberrations of two sextupoles separated by a $-I$ module is first shown. Then the more complex example of a fourth order aberration, i.e. a fifth order Hamiltonian, is discussed. The basis for these elaborate manipulations is the similarity transformation. A discussion on the errors and induced effects ends the chapter.

The second part of the thesis consists in an application of the methods to the case of the Final Focus Test Beam. The aberration content of the FFTB at order up to five in the Hamiltonian, or fourth order optical effects, is analyzed in chapter 4 . The approach is here analytical and since the number of potential aberrations is rather high, some arguments are derived in order to reduce the number of terms that actually need to be estimated. Several arguments are invoked here, including one that uses the characteristic phase advance pattern of final focus systems. The aberrations are then studied order by order. I actually make a distinction between two types of aberrations. Those that are present by design, in the perfect 
line, are called inherent aberrations while those that arise because of some cror (position, strength, etc.) are called induced aberrations. The orders quoted in the next paragraphs are the orders in the Hamiltonians which is one more than the actual optical order.

Since we have already removed the design linear optics as explained in chapter 3 , the only aberrations present at first and second order are induced. At higher order inherent and induced aberrations are mixed. The FFTB is corrected at third order except for one identified term for which a correction is proposed. The significant fourth and fifth order aberrations are then reviewed. Finally I show how the aberration pattern at the FFTB is closed at fifth order. That is there cannot be a significant aberration at order higher than five.

Finally the last chapter reviews some stability tolerances for the FFTB. Although Lie algebra is not needed for this mostly first order study, it shows that it is possible to treat linear problems in a very simple way using these methods also. The individual tolerances are derived for the position stability of the magnets. The effects of steering at the IP as well as the dispersion, normal and skew quadrupole effects induced by the subsequent orbit displacement are reviewed. The tolerances on strength stability and rotation are also derived. Finally the tolerances on the sextupolar harmonic content of quadrupoles is also given.

Throughnut this thesis I will freely use without further definition some basic concepts of accelerator physics such as the betatron functions, the phase advance, etc.. The notations are those in use in the United States. The orientation of the axes follows also the american convention of the $y$ direction being in the transverse plane and pointing upwards. Directions $z$ and $s$ are the longitudinal axes in respectively the cartesian and curvilinear coordinate systems. 


\section{The Optics of the Final Focus Test Beam}

\subsection{INTRODUCTION}

I review in this chapter the design and performance of the optics of the FFTB which was based upon preliminary work ${ }^{[5]}$ by Katsunobu Oide during his one vear visit to SLAC and upon the experience ${ }^{[2]}$ gained at the Stanford Linear Collider (SLC).

After some general background on the focusing and chromatic correction in final focus systems, I describe the layout of the FFTB and the optical functions of each module as well as the associated instrumentation. The history of the FFTB design, both the early choices and the evolution of the present design, is reviewed in the third section.

Finally I address the issue of the performance of the optics in terms of the final spot size, the energy bandwidth of the system and limiting factors such as remaining aberrations. A few of these aberrations are developed here in detail. Tnlerances, altho'tgh an important part of the performance of the line, will be reviewed in a later chapter when we have more powerful tools available.

\subsection{Final FOCUS SYSTEMS}

\subsubsection{Telescopes}

The focusing of a particle beam can be achieved by using simple quadrupolar magnetic lenses. A basic one dimensional optical system commonly used to demagnify the transverse dimension of a beam is a telescope such as the one shown here:

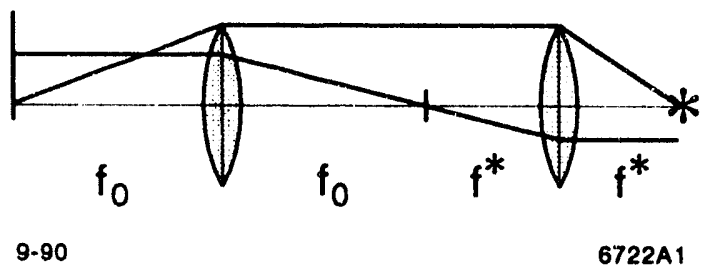

Figure 1.1. One dimensional telescope made of two quadrupole lenses and showing the sine and cosine-like principal trajectories. This telescope achieves a point-to-point and parallel-toparallel focusing in one plane only. 
This simple telescope achieves a point-to-point and parallel-to-parallel imaging together with a geometric demagnification $\frac{1}{M_{x}}=\frac{f_{0}}{f^{*}}=\left(\frac{\beta_{0}}{\beta^{*}}\right)^{1 / 2}$ where $\beta_{0}$ and $\beta^{*}$ are the values of the betation function ( $\beta$-function) at the entrance and at the focal point of the telescopic system. The one dimensional first-order matrix representation of such a moclule is:

$$
R_{x}=\left(\begin{array}{cc}
-M_{x} & 0 \\
0 & -1 / M_{x}
\end{array}\right) \text {. }
$$

In order to achieve the same effect in both horizontal and vertical planes one needs a minimum of four lenses. Other solutions can be based on triplets instead of cloublets and are particularly well suited to the case of round beams with equal emittances like those of the SLC. ${ }^{[1]}$

One can then demagnify a beam, generating a very small $\beta$-function function as rhe IP and, assuming a monoenergetic beam with emittance $\epsilon$, no dispersive contribution and no coupling, the linear beam size $\sigma_{0}^{*}$ at the focal point, is given by: $\sigma_{0}^{* 2}=\epsilon \beta^{*}$.

\subsubsection{Chromaticity}

A beam having a finite energy spread imaged by quadrupolar magnetic lonses laas the adverse property that particles with different momenta are focused at different clistances; parti les with a higher momentum $\left(p=p_{0}(1+\delta)\right)$ come to a focus at a point downstream of the nominal focal point and those with lower momentum are focused upstream. The system thus has chromatic aberrations at the focal point.

The spot size at the end of a beamline containing quadrupoles and other chromatic elements, such as the telescope of fig. 1.1, and for a beam with some energy spread, is given by

$$
\sigma^{* 2}=\sigma_{0}^{* 2}\left(1+\xi^{2}\langle\delta\rangle^{2}+\ldots\right)
$$

The chromaticity $\xi$ is defined as

$$
\xi_{x, y}=\int_{s_{0}}^{s_{1}} K(s) \beta_{x, y}(s) d s
$$

where $K(s)=\frac{B_{0}}{a\left(B_{0}\right)}$ is the strength of the quadrupoles along the line. The chromaticity is a measure of the strength of some chromatic aberrations at the focal point. 
For a given lattice the chromaticity is inversely proportional to the $\beta$-function at the focal point. Equation (1.2) then shows that as one decreases the $\beta$-function at the focal point the spot size passes through a minimum after which it increases again due to chromatic aberrations ( $c f$. figure 1.7).

For final focus systems the most important contribution to the chromaticity, up to $90 \%$, comes from the final quadrupole doublet (or triplet) where both the $\beta$-functions and the quadrupole strengths are large.

This results in an increase of the spot size unless one can bring the total chromaticity of the line close to zero. The remaining third and higher order chromatic as well as geometric aberrations then limit the performance of the line.

\subsubsection{Chromaticity correction}

A solution to correct the chromaticity (the energy dependent focusing from the quadrupoles) is to introduce sextupoles in the optics in a region where there is horizontal dispersion $(\eta)$. A sextupole is a magnet with a focusing strength varying linearly with the horizontal distance to the axis. Therefore placing a sextupole in a linear dispersive region focuses the particles with a strength proportional to their energy. The sextupole integrated strength

$k_{s}=\frac{B_{0}}{a^{2}(B \rho)} l_{s}$, dispersion and $\beta$-function at the sextupole can be adjusted so that the cinromaticity introduced $\left(\xi_{s}=k_{s} \eta \beta_{s}\right)$ is exactly opposite to that $\left(\xi_{q}\right)$ produced by (mainly) the last quadrupoles. The optimum location for the sextupole is $\pi$ in phase upstream of the final quadrupoles.

A sextupole also introduces strong second order geometric aberrations which would dominate the spot size. The cancellation of these dangerous geometric aberrations is achieved by inserting the sextupoles in pairs separated by a $-I$ transformation (a $\pi$ phase advance module with a magnification of one). The second-order geometric aberrations then cancel due to the even parity of the kick given by sextupoles, while we can malie the chromaticity of the sextupoles add by manipulating the dispersion function in the $-I$ transform so that the chromatic kick has an odd parity.

Because the horizontal spot size is small enough to be aberration sensitive, we also have to correct the chromaticity in the horizontal plane. 
This schene which was first introduced for the second order achenat ${ }^{[2,51]}$ by Brown and Servanclix is the basis of chromaticity correction for final focus systems and has already been successfully used at the SLC although, because of severe space limitations, the horizontal and vertical chromatic correction sections were interlaced in the SLC while they are separated at the FFTB. This scheme requires the introduction of sextupoles and bending magnets which in turn create third and higher order aberrations which limit the ultimate performance of the system.

\subsection{FFTB OPTICS}

The Final Focus Test Beam is located in a straight-ahead channel at the end of the SLAC linac, the C-beam line ( $c f$. figure 1.2). The basic parameters of the FFTB were chosen as close as possible to potential parameters of a future linear collider ${ }^{[5]}$ The gradient of the final quadrupole is $G=1.8 \mathrm{~T} / \mathrm{cm}$ with a half-aperture $a=6.5 \mathrm{~mm}$. The $\beta$-functions at the focal point are $\beta_{x}^{*}=3 \mathrm{~mm}$ and $\beta_{y}^{*}=100 \mu$. The available transverse and longitudinal space in the Beam Switch Yard/Research Yard and the shielding requirements ${ }^{[3]}$ were the major physical constraints affecting the design. The total length of the FFTB is 392 meters from the end of the linac to the dump with 185 meters of that amount from the entrance of the FFTB ( $\beta$-matching quadrupoles) to the final focal point.

The FFTB will use the $50 \mathrm{GeV}$ electron beam from the SLAC linac operated in a flat beam configuration, with unequal emittances $\epsilon_{y} \leqslant \epsilon_{x}$. There is no provision to use the positron beam unless one changes the polarity of all the magnets which can be done by switching the leads on all the power supplies. In the "decoupled tunes" modr of operation, the SLC damping ring can deliver a beam with vertical invariant emittance ${ }^{[24]}$ of $\gamma \epsilon_{y}=$ $7 . \times 10^{-7}$ m.rad. This corresponds to a theoretical linear spot size of $27 \mu \mathrm{m}$ for $\beta_{y}^{*}=100 \mu$. We believe the emittance growth taking place in the linac can be controlled so as to deliver $\gamma \epsilon_{y}=3 . \times 10^{-6}$ m.rad. at the entrance of the $\mathrm{FFTB},{ }^{[25]}$ in order to achicve a vertical spot size of $60 \mathrm{~nm}$, at the focal point. The horizontal emittance $\gamma \epsilon_{x}=3 . \times 10^{-5}$ m.rud. together with $\beta_{x}^{*}=3 \mathrm{~mm}$ gives a horizontal spot size of about one micron at the focal point.

By coupling the horizontal and vertical damping ring optics and resetting five quadrupoles at the entrance of the FFTB line, a round spot (down to $1 \mu \times 1 \mu$ ) can be produced at the focal point. This configuration will be especially useful in the early tuning stages when a spot size compatible with standard measuring techniques (wire scamers) is desirable as 


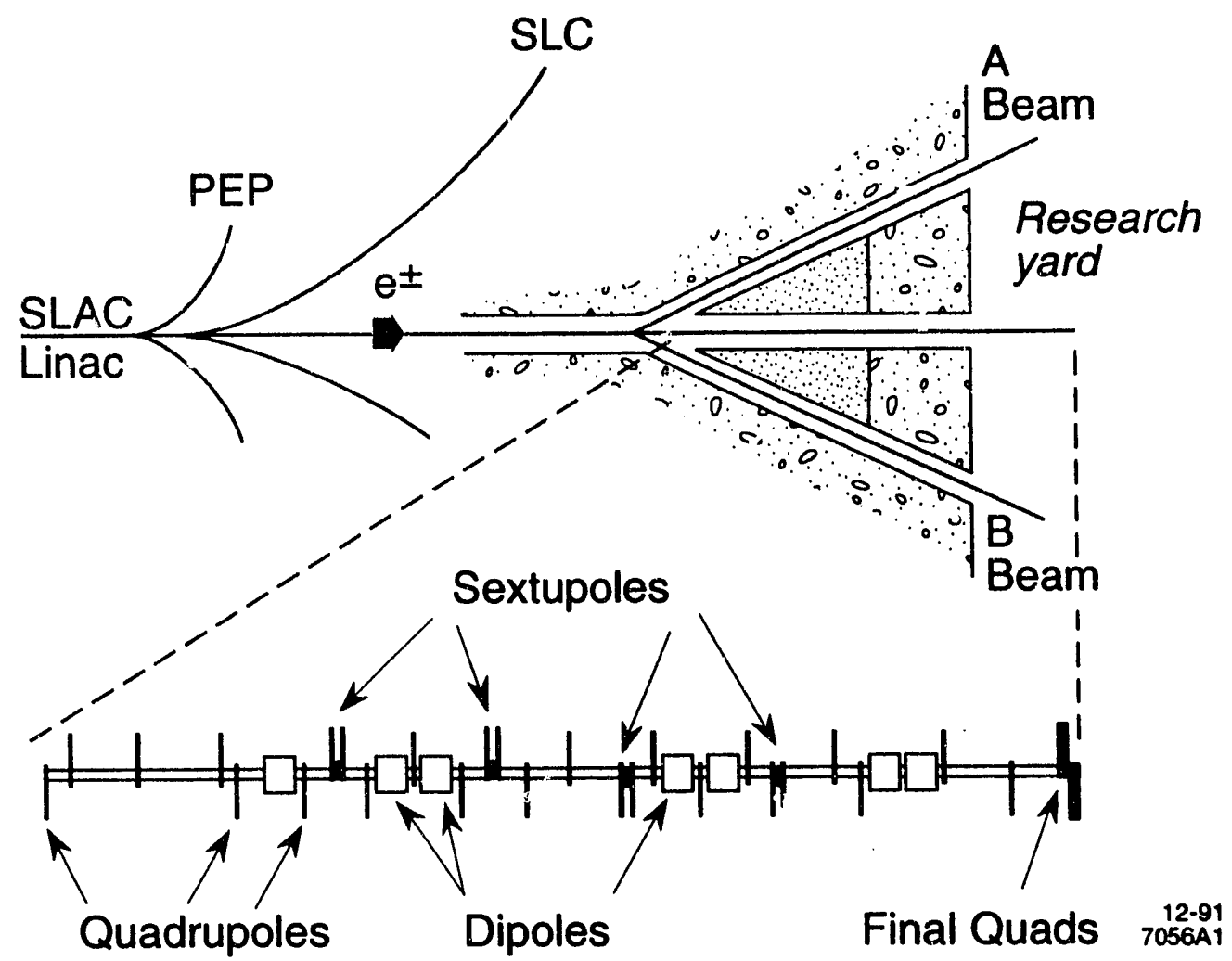

Figure 1.2. FFTB layout schematic showing the relation of the FFTB locati in (C. Line) with respect to the SLC arcs as well as tie $A$ and $B$ beamlines.

we will need to calibrate the new beam size monitor ${ }^{[37]}$ against known techniques. Another configuration of the first five quadrupoles is proposed for the purpose of making emittance and $\beta$-function measurement at the entrance of the line.

\subsubsection{Optical modules}

I now turn to a description of the FFTB optics itself. It is convenient, both for the design and the operation of such a line, to decompose it into optical modules, each having a specific function and being independent of the others for tuning and correction purposes. An example of a telescopic module was given in the first section of this chapter. The FFTB is composed of five optical modules. Starting from the IP and going back towards the linac we find the Final Transformer (FT), the vertical Chromatic Correction Section (CCY), a Beta Exchange section (BX), the horizontai Chromatic Correction Section (CCX) and finally the Beta Matching area (BM).

A characteristic of final focus optics is the alternating very large and very small $\beta$ functions. Large values, often several kilometers, occur at the final quadrupoles and are 
nerred to obtain the large divergence that lead to a small final spot size. The chromaticity correction also requires high $\beta$-functions at the sextupoles. The required phase advance between sextupoles and between the last sextupole and the final quadrupoles imposes that. the $\beta$-functions also go through very small beta points where most of the phase advance is actually concentrated. There is a typical pattern for the phase advance in final focus systems: The phase is quasi-constant everywhere except around the low beta points where it jumps suddenly by $\pi$. Most elements in a final focus lattice are therefore in phase with each other, more exactly separated by $n \pi$ in phase. Only a few elements are placed at the opposite phase, separated by $\pi / 2+n \pi$ from most elements.

Natural boundaries for the different modules are conveniently placed according to this phase pattern. The most important elements of the system are the four sextupoles and the last quadrupoles. The four sextupoles constitute the boundaries that define the five optical modules at the FFTB.

\subsubsection{Final Transformer}

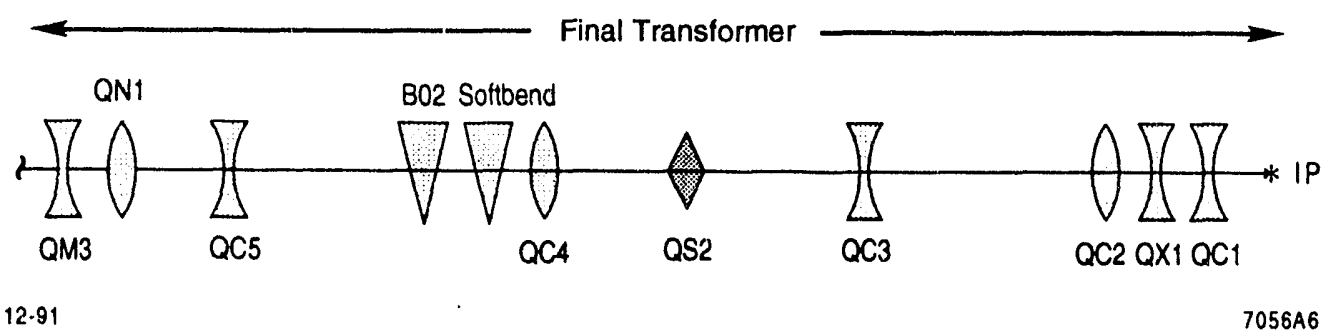

Figure 1.3. Schematic of the FFTB Final Transformer. The beam goes left to right to the IP. Quadrupoles are figured as schematic optical lenses, bending magnets as wedges and a skew quadrupolè is shown as a lozenge.

The Final Transformer (FT) achieves the demagnification of the beam. Although it is not by itself a telescopic module, the entire line forms a telescope so that the first order transformation matrix between the end of the linac ("swivel point") and the focal point does have the form shown above with the demagnifications $1 / M_{x} \approx 70$ and $1 / M_{y} \approx 380$. These values are similar to those required for a final focus system for the next generation of linear colliders. For a comparison the SLC demagnifications ${ }^{[1]}$ are $1 / M_{x}=42.5$ and $1 / M_{y}=15.5$.

The chosen arrangement allows a slight reduction of length compared to a final forus system based on a rigorous telescopic arrangement. There is however a penalty for this less symmetric design; there are no casily accessible image points of the IP in the line. 
The final transformer can be subdivided into two ensembles of magnets. The last three magnets before the IP (QC2 - QX1 - QC1) are commonly referred to as the "final doublet" since QC1 and QX1 have the same polarity, opposite to that of $\mathrm{QC} 2$. These magnets are being designed and built by KEK (Japan). They actually form a rigid block mounted on a single stand, also conceived and provided by the Japanese laboratory, with its own stabilization system. These magnets are very strong with a maximum of $14 k$ Gauss for QX1 equivalent to a gradient of $1.4 \mathrm{~T} / \mathrm{cm}$. The maximum gradient, $G=1.8 \mathrm{~T} / \mathrm{cm}$ is achieved in QC1. The history of this doublet is reviewed in a later section.

The other magnets in this module are more conventional and provided by the Institute for Nuclear Physics in Novosibirsk (Russia, formerly USSR) from a joint SLAC/INP design. The frontier between the final transformer and the vertical chromatic correction section is set at the last sextupole. There is therefore a total of eight quadrupoles in the FT including the last three forming the "doublet".

There are also two groups of bending magnets in the FT. The last group (B03) consisting of two bending magnets forms the so-called "soft-bend" with a field about one tenth of that of the main bends. They are designed to sweep the beam away from the hard synchrotron radiation originating in the main bends.

The first group of bending magnets bring the dispersion function back to zero before the Interaction Point. Note that there is not really an Interaction Point at the FFTB since we have only one beam. The Focal Point which is really the Interesting Point in our case, is denoted IP.

Because the last leg of the FT is dispersion free it is also a natural place for instrumentation and correctors. We have the following corrector magnets in the Final Transformer: one skew quadrupole, two sextupoles and two skew sextupoles. In addition we have a total of nine (four horizontal and five vertical) dipole correctors located at both $\frac{\pi}{2}$ and $\pi$ phase advance away from the IP. They provide for steering and dispersion correction at the IP. 


\subsubsection{Chromatic Correction Sections}

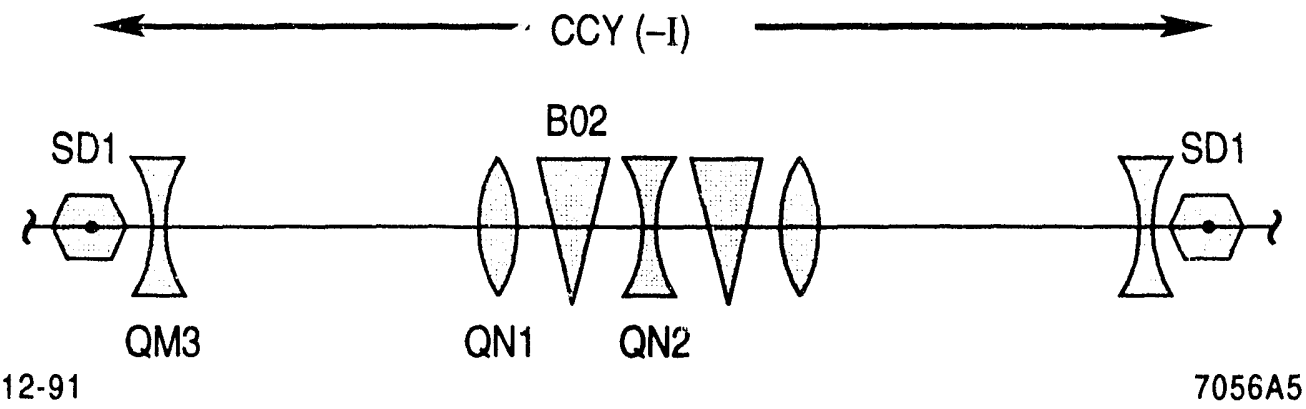

Figure 1.4. Schematic of the FFTB Chromatic Correction Section. The section has a mirror symmetry around the central quadrupole, QN2 here. Quadrupoles are figured as schematic optical ienses, bending magnets as wedges. Sextupoles are drawn as hexagons.

The two chromatic correction secitions are so similar in design that I will clescribe them together. In fact they are identical except for the sign of the quadrupole strengths; where the vertical CCS (CCY) has a focusing quadrupole, the horizontal one (CCX) has a defocusing quadrupole of same absolute strength. The horizontal and vertical chromaticities can be tuned separately. This two-plane correction scheme increases the total length of the system and the number of components required but is essential in order to obtain the small spot sizes in both planes at the focal poi. $t$.

The CCS is brilt from a modified FODO cell with a mirror symmetry about the central magnet. There are a total of five quadrupoles in each CCS and two groups of two bending magnets around the central quadrupole. The $\beta$-function is matched to be at a maximum $(\alpha=0)$ at the sextupoles and at a minimum in both planes at the center of the central quadrupole, making these points (one in CCX and one in CCY) the only true image points of the IP at the FFTB.

Being located inside the magnets the image points are not available to instrumentation such as wire scanners. Even if they were available, they could not be used in the nominal $(1 \mu \times 60 \mathrm{~nm}$ at the IP $)$ configuration since the spot size is clominated there by chromatic aberrations originating in the first sextupole of the pair. 


\subsubsection{Beta Exchanger}

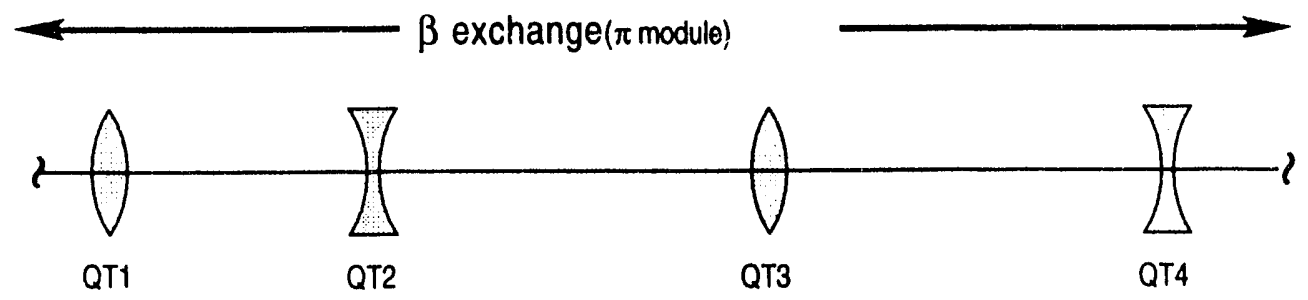

$12-91$

7051.44

Figure 1.5. Schematic of the FFTB Beta Exchanger. The beam goes left to right . Quadrupoles are figured as schematic optical lenses.

Between the two CCS is a " $\beta$-exchanger" to manipulate the $\beta$-function from one section to the other. It is also a $\pi$ phase advance module consisting of four leuses arranged in a modified FODO lattice. The instrumentation in this section consists of two wire scanners at the points where the $\beta$-functions are at a minimum. These points have very large aspect ratios, i.e. the beam is very elongated in either the vertical or the horizontal direction: $\sigma_{y} / \sigma_{x}=36$ at the $\mathrm{X}$ waist and $\sigma_{x} / \sigma_{y}=376$ at the $\mathrm{Y}$ waist. This can present some difficulties for the design of the wire scanners. We also have a total of four correctors in this section. They are designed to launch an orbit into the CCY in both position and angle as well as close an orbit launched across the CCX by correctors in the beta matching section.

\subsubsection{Beta Matching Section}

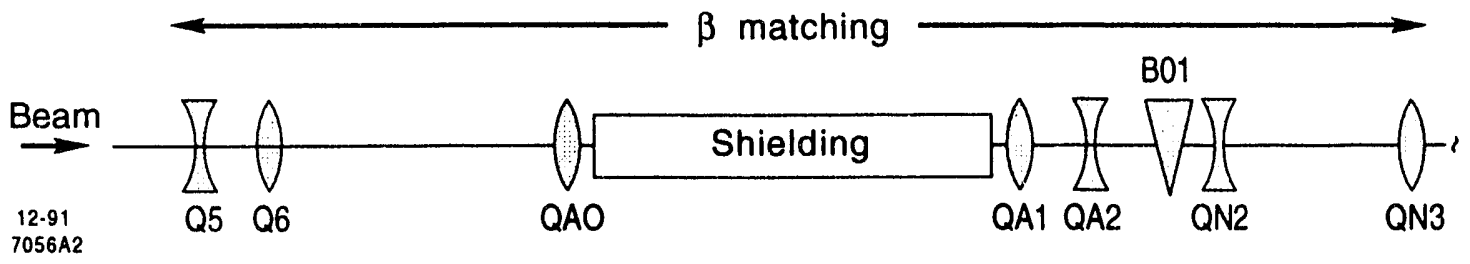

Figure 1.6. Schematic of the FFTB Beta Matching section. The beam goes left to right. Quadrupoles are figured as schematic optical lenses, bending magnets as wedges. Also figuring is the 16 meter-long muon shielding wall.

Finally, before the first CCS, we have a beta matching section which extends from the end of the divergence chamber (dump D10) to the first sextupole in the line. For lack of space 
it was not possible to design the nominal configuration to include in this section an image point where the incoming beam could be characterized using a screen or a wire scanner. We had to resort to a different configuration of the beta matching section for this purpose, the so called "matching configuration" as opposed to the "nominal configuration". In matching configuration, the five quadrupoles are reset so as to form a waist in both planes at a wire scanner (just upstream of B01) where the incoming beam can be analyzed. The quadrupoles are then set in order to match the incoming beam to the rest of the lattice. When operating in the matching configuration, the beam is stopped before entering the CCS.

\subsubsection{Dump line}

The dump line extends from the IP to the dump itself. It has three major functions: the capture of the beam after the focal point; the safe guiding of the beam to the clump and finally the measure of the relative energy of the beam.

Because of the very large divergence of the beam at the IP $\left(\sigma_{y^{\prime}}^{*} \sim 500 \mu \mathrm{r}(\mathrm{d})\right.$ ) and because we want to allow for a reasonable space for instrumentation around the IP, the capture is not trivial as the beam size increases very rapidly. A total of eight quadrupoles arranged in five optical lenses are used. These quadrupoles are of a design similar to that of the other quadrupoles of the FFTB but they have a much larger aperture. They are not mounted on magnet movers and are not fitted with BPMs. After the quadrupoles one finds four vertical bending magnets that deflect the beam towards the ground. The magnets are also derived from the main bending magnets of the FFTB. They are conventional iron magnets although they may be replaced by permanent magnets for beam containment as well as experimental reasons. ${ }^{[26]}$ A shiclded dump is located some thirty meters downstream of the last magnet. The vertical deflection creates vertical dispersion which is used to measure thr (relative) energy and energy spread of the beam. ${ }^{[3]}$

\subsection{History OF THE DESIGN}

\subsubsection{Early choices}

The FFTB design has evolved from a solution derived by Oide. Another solution was derived by $K$. Brown and R. Helm and was based on the SLC final focus model with cloubletbased, strictly modular telescopes and two non-interleaved chromatic correction sections. 
Those CCS were actually $2 \pi$ phase advance modules (with of course the $-I$ betweer sextupoles). The two beamlines were performing in a very similar way; same final spot sizes and similar bandwidth. The line by Brown and Helm had actually more clements than the design by Oide and was also longer. These were the two major reasons why the decision was made to base the FFTB on the optics designed by Oide.

The final focus lattices now being studied for the Japan Linear Collider (JLC) are based on the same ideas from Oide while those for the Next Linear Collider (NLC) developed by Brown and Helm apply the same rigorous modular principles that were proposed for the FFTB. A comparison of two such lattices on the basis of tolerances was done ${ }^{[23]}$. The complete study of tolerances for the chosen FFTB lattice is given in chapter 5 .

\subsubsection{Evolution of the present solution}

After the choice of lattice was made, there were a number of important milestones in the development of the optics as it is now being implemented. Let me comment more on four of these changes:

First the original final doublet using two quadrupoles was changed for a three quadrupoles design. This solution which splits the final quadrupole into a short $(30 \mathrm{~cm})$ and very strong first magnet followed by a weaker but longer $(110 \mathrm{~cm})$ second magnet was prompted by engineering and costs reason. Since this last lens has to accept a large beam and focus it very strongly the beam size varies greatly along the magnet. The aperture must be calculated to allow for clearance at the entrance while there is extra clearance at the end when the beam is already strongly focused. An idea of using a tapered magnet where the aperture would be gradually reduced could be a solution to this problem. In our case it was cuough to split the magnet in two parts. The modification to the rest of the lattice was minimal. The two quadrupoles forming the last lens will be strongly tied together and should for all tolerances purposes form one unit.

Another change in the optics was prompted by magnet engineering reasons. At a design field of nearly $10 \mathrm{kGauss}$ the original $18 \mathrm{~cm}$ long sextupoles were experiencing some saturation of the iron and we had to increase their lengths to $25 \mathrm{~cm}$ in order to stay within some reasonably linear region. The change in this case was of potential importance to the design since the long sextupole effect is a well known aberration of the system and it was taken into account for the optimization of the design by Oide. Although the change did seriously 
increase this octupole-like aberration, it is still at a manageable level. I will expand on this in the next section of this chapter and a detailed calculation is in Appendix A.

Related also to the departure from Oide's optimized parameters is the change in bending angle of the main dipoles. The optimum value as calculated by Oide ${ }^{[5]}$ was 3.5 mrad per bending magnet for the original lattice. The bending angle is now $3.7 \mathrm{mrad}$ per magnet. The consequence of the modification is a slight increase in two effects caused by the synchrotron radiation in the bends. The growth of the horizontal emittance and the chromaticity unbalance are detailed in Appendix A: In either case the difference is small enough that there is no impact on the performance of the line.

The most important change in the design was to "square the lattice". This consisted in two operations. First the optics was $m$. .tched so that $\alpha_{x}=\alpha_{y}=0$ at the sextupoles. This is not a necessary condition for the chromaticity correction but is helpful for the tuning ${ }^{[45]}$ of the optics. The second change was to match the dispersion function so that we also have $\eta^{\prime}=0$ at the sextupoles. Since the dispersion can be seen as a particular trajectory in the horizontal plane, the dispersion function and the horizontal $\beta$-function now follow a similar pattern and there is a waist at the mid-point of the first and last group of bending magnets where the dispersion is launched and cancelled. Additional dipole magnets manipulate the dispersion inside the CCS. The maximum value of the dispersion function is now reduced by a factor two compared to the original design. The operation of "squaring" the lattice was prompted by the development of the tuning and correction procedures. It does help the tuning of the line by simplifying the optics but is not necessary from a point of view of purely optical design.

\subsection{Performances}

\subsubsection{A measure of final focus performance}

The measure of the performance of a final focus system can be approached from at least two different sides. Of course it all comes down to one simple precept: the smaller the aberrations, the better the system performance. In general the idea is to have a system that not only delivers a very small spot at the interaction point but also delivers this spot over an energy bandwidth as large as possible. For most designs of linear colliders an energy bandwidth of 0.3 to $0.4 \%$ seems adequate, however some designs, VLEPP for example, may require a larger bandwidth. 
The approach at the FFTB was to consider a fixed energy spread of $\pm 0.4 \%$ and design the system to achieve the magnifications anticipated for an NLC as we have already mentioned. The performance of the system in that case can be measured by the remaining aberrations at the focal point when the lattice is tuned and corrections (chromaticity, dispersion coupling, ... ) have been applied. I express this as the ratio $p$ of the real spot size, obtained here from tracking, to the linear spot size $p=\sigma_{y}^{*} / \sqrt{\beta_{y}^{*} \epsilon_{y}}$. For the FFTB, $p=60 / 55=1.09$ indicating that the uncorrected aberrations amount to about $9 \%$ of the linear spot size. This value of course depends on the lattice. By varying the demagnification of a given lattice (using only the matching quadrupoles) we get the so-called performance plot on figure 1.7 showing the evolution of the final vertical spot size with the vertical $\beta$-function at the focal point in the following cases: linear $\left(\sqrt{\beta_{y}^{*} \epsilon_{y}}\right)$, uncorrected showing the effect of the chromaticity of the lattice (sextupoles are turned off) and chromaticity corrected (sextupoles on). The curve for the corrected case is the one we hope to observe at the FFTB. It shows the effect of higher-order uncorrected aberrations.

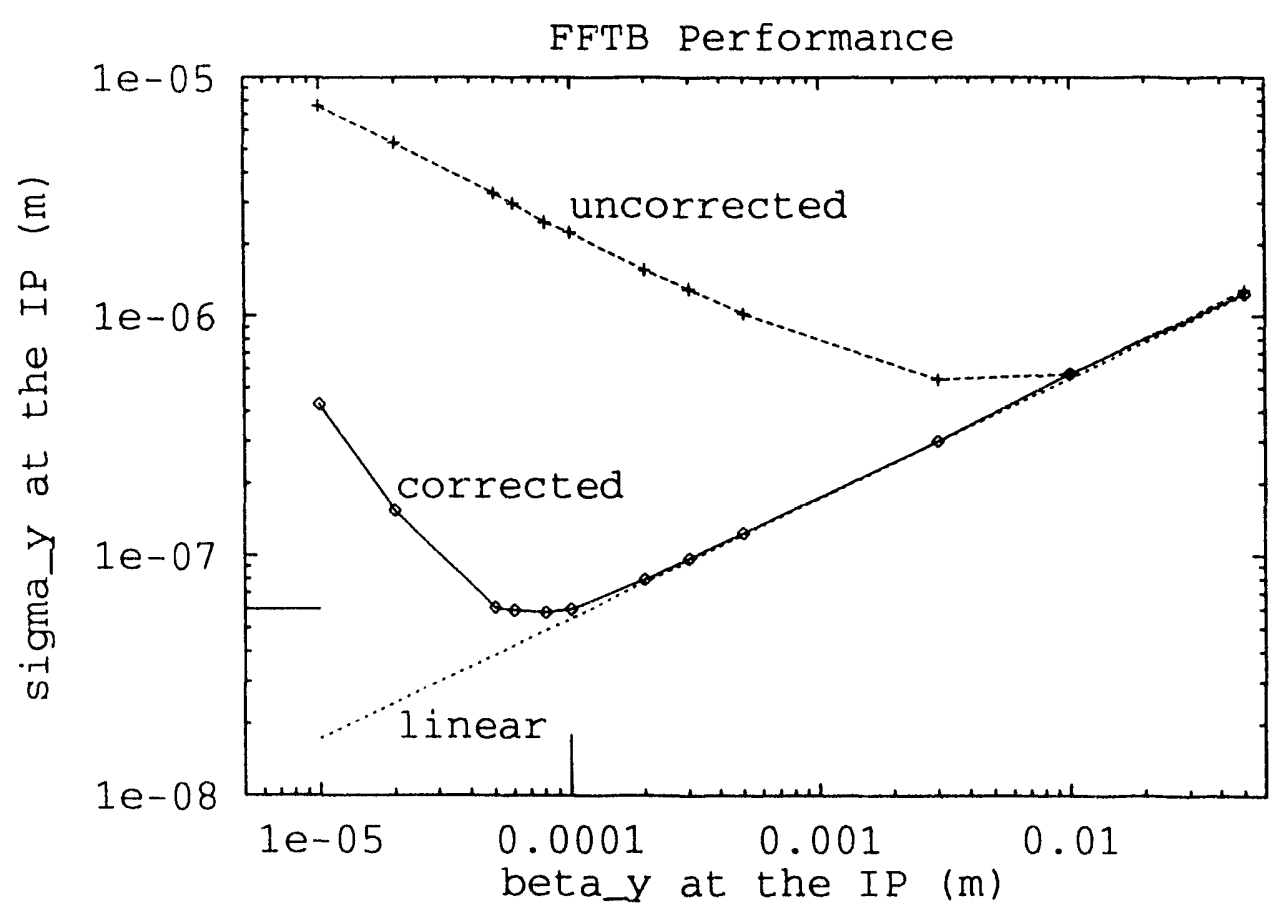

Figure 1.7. FFTB Performance plot. The linear curve shows the linear spot size at the IP as a function of the $\beta$-function. The uncorrected and corrected curves show the result of tracking with respectively sextupoles off and on. The chromaticity correction is the main clifference between the two. Remaining higher-order aberrations are shown to be significant below the nominal $\beta$-function of $100 \mu$. 
Another facet of final focus performance is the energy bandwilth of the system, or the energy domain over which the chromatic correction is effective. Some effort has been placed lately on final focus systems with large bandwidth such as the one by R. Brinkmam at $\mathrm{DESY}^{[27]}$; using eleven sextupoles strategically placed and tuned, he could increase the bandwidth of his beamline to about $\pm 2 \%$. The optimization was clone using a computer fitting code. The principle of the correction is primarily the cancellation of the local chromaticity at the quadrupoles although the geometric effects from the sextupoles as well as higher order chromatic aberrations are of importance. This approach could shift the difficulty of a linear collider from the delivery by the linac of a beam with small emittance and small energy spread to a likely problem of tolerances (strength, position) in the final focus. The question of the tuning of eleven sextupoles is also important.

There are however other concerns here besides ensuring that the energy acceptance of the final focus matches the energy spread of the beam at the end of the linac. The only strong reason to design a large bandwidth system might be the barkground in the detector. Studies at the SLC show that this is a strong reason for the limitation of the luminosity clelivered to the detector. Also some physics studies such as the top quark search require that the center of mass energy of the collisions be well defined and have a narrow distribution. In most cases this requires that the energy spread delivered by the linac be small, alleviating the demand for a large bandwidth in the final focus.

More studies of the type of solution presented by $R$. Brinkmann should certainly be carried out especially to find the associated tolerances as well as understand more precisely what type of cancellation is taking place, what aberrations disappear and through which mechanism. The Lie algebra methods I present in this thesis seem adapted to this type of stindy.

\subsubsection{Residual aberrations - limits on the spot size}

The three major remaining aberrations have been identified at the FFTB by Oide and others. They are, in the order they are presented in this section, the chromaticity unbalance due to synchrotron radiation, the long-sextupole aberration and the chromatic breakelown of the $-I$ of the CCS. They are simply outlined here but are detailed in Appendices $A$ and $B$ for the first two and in another chapter for the last one. 


\section{- Chromaticity unbalance}

The bending magnets are a source of optical distortions, ${ }^{[14,49]}$ both geometric and chromatic. However the bending angles are small enough that the geometric effects are negligible in our case. The major effect of bending magnet is a chromatic one through synchrotron radiation: We have seen that the principle of chromatic correction is the cancellation, by sextupoles, of the energy dependent focusing of the quadrupoles. However as particles pass through the bends between the sextupoles and the final quadrupole, they lose some energy through synchrotron radiation. These bends are needed to bring the dispersion back to zero at the focal point. The canceliation between the chromaticity introduced by the sextupoles and that introduced by the final quadrupole can no longer be completely rigorous as a result of the quantum fluctuations in the synchrotron radiation. There is an increase of the spot size at the IP due to uncorrected chromaticity. This has been calculated by K. Oide and I redevelop it in detail in appendix A. The result for the FFTB configuration with bending magnets of length $l_{b}$ and angle $\theta_{b}$, is:

$$
\Delta_{b}^{2}=\frac{\Delta \sigma_{y}^{* 2}}{\sigma_{y}^{* 2}}=\frac{55}{16 \sqrt{3}} \cdot r_{e} \lambda_{e} \gamma^{5} \frac{\theta_{b}^{3}}{l_{b}^{2}} \xi_{y}^{2} .
$$

For the FFTB parameters we have $\Delta_{b}^{2}=.06$ which corresponds to an increase of the spot size of $\sim 3 \%$.

\section{- Long-sextupole}

The second most significant source of higher-order aberrations is the sextupole length which generates third order octupole-like geometric aberrations. It can be explained by mentally cutting the sextupole into elementary slices. The kick given by a slice of the sextupole is a non-linear function of the transverse position of the particle at that location. The position of the particle at any slice is affected by the kicks given by preceding slices of the same sextupole. The total effect departs from the simple sum of the individual kicks since they are non-lincar. The difference is the long-sextupole effect, a third-order, octupole-like, aberration. In terms of spot size increase at the focal point we have, as cleveloped by Oide and as described in Appendix B:

$$
\Delta_{s}^{2}=\frac{\Delta \sigma_{y}^{* 2}}{\sigma_{y}^{* 2}}=\frac{5}{12} \cdot k_{s}^{4} l_{s}^{2} \epsilon_{y}^{2} \beta_{y}^{4}
$$

where $k_{s}$ is the integrated strength of the sextupole. For the FFTB, $\Delta_{s}^{2}=.06$ corresponding 
also to an increase of the vertical spot size of the order of $3 \%$.

Combining these first two effects, one sees that we have to optimize the sextupoles and bending magnets in order to minimize $\Delta_{0}^{2}=\Delta_{s}^{2}+\Delta_{b}^{2}$ given the available length for the system. This was done by K. Oide for the $\mathrm{FFTB}^{[3]}$ and gives $\Delta_{0}^{2}=.12$ for the present design. This corresponds to an increase in the spot size of $6 \%$. Tracking results, including syuchrotron radiation simulation, show that the spot size is about $60 \mathrm{~nm}$ or about $10 \%$ above the design linear spot size of $55 \mathrm{~nm}$.

\section{- Chromatic breakdown}

There is indeed another aberration giving an effect of the same order of maguitude as the first two. It is caused by the chromatic breakdown of the CCS coupled to the chromaticity of the final quadrupoles. The details of this aberration will be worked out, in a slightly different way, in chapter 4 but I can give here a qualitative explanation for it.

The cancellation of the purely geometric terms from the two sextupoles of each CCS relies on the fact that there is a $-I$ transformation between them. Since the quadrupoles inside the CCS introduce some chromaticity, the $-I$ is not true for off-momentum particles. Therefore there is a residual chromo-geometric (third order in the optics) effect at the end of the CCS. This effect however is small since the chromaticity coming from the central quadrupole of the C'CS is also small thanks to the very smali $\beta$-function at this point. However Oide has identified that this term can actually generate a significant effect when one considers its coupling with the very strong chromaticity of the final quadrupoles. The chromo-geometric term changes the position of the particles in the final quadrupoles, changing the chromatic kick they receive. This chromatic kick no longer cancels exactly the one received in the sextupoles.

This fourth order effect is of magnitude similar to that of the long sextupole effect. This example shows how one should be careful in analyzing these aberrations; some apparently negligible effect can feeddown with a big effect downstream to generate some sizable effect. overall. However this feedrlown can only appear at a significant level because of the presence of a very large term such as the final quadrupole chromaticity. The complete treatment of this aberration is presented differently in chapter 4 but this is the way Oide presented it the first time. Oide used a simplified model to derive the expression of this aberration. A rigorous expression is presented in chapter 4 using Lie algebra based terhuicpues. 


\subsubsection{Additional effects}

The aberrations we have considered here are the main limitations of our system in the vertical plane. One could reduce the strength of the first two by significantly increasing the length of the system in order to reduce both the sextupole strength and the dipole bending angle but there is a tradeoff between this reduction and the available space on the SLAC site.

Another effect of the bending magnets is the increase of the horizontal emittance of the beam caused by quantum fluctuations in the synchrotron radiation process. ${ }^{[5 \cdot]}$ The calculation for the FFTB shows that this effect increases the horizontal emittance by $2.7 \%$. Combined to an increase of the $\beta$-function of the same order, this leads to a $1.3 \%$ increase in the horizontal spot size. However this does not affect the vertical emittance in the absence of coupling, thereby leaving the vertical spot size at the focal point untouched.

Because the last quadrupole is so strong and the beam is large at that location before being strongly focused to the final focal point, the paricles can emit a significant amount of synchrotron radiation while passing through the final quadrupole, leading to esseutially the same process we have described for the bends: the chromaticity the particle experiences is slightly different from what the sextupoles correct for, leading to some unbalance in the chromaticity correction. This effect, which has become known as the "Oide effect" sets an ultimate limit for the smallest spot size that can be achieved. The result depends almost only on the normalized emittance $\gamma \epsilon_{y}$ :

$$
\sigma_{y \text { min. }}^{*}=\left(\frac{T}{5}\right)^{\frac{1}{2}}\left[\frac{275}{3 \sqrt{6 \pi}} r_{e} \lambda_{e} F\left(\sqrt{\Gamma} l_{Q}, \sqrt{\Gamma l^{*}}\right)\right]^{\frac{1}{7}}\left(\jmath \epsilon_{y}\right)^{\frac{5}{7}}
$$

The function $F$ is a dimensionless function of the length and strength of the final quadrupole and the focal length of the system. ${ }^{[54]}$

In the case of the FFTB, $\sigma_{y \text { min. }}^{*}=30 \mathrm{~nm}$ while we aim at obtaining $\sigma_{y}^{*}=60 \mathrm{~nm}$. This effect is expected to be significant for future linear collider finat focus systems where extremely small vertical spot sizes are needed. 


\subsubsection{Higher order investigation}

The formulas describing the aberrations of a line can certainly be obtained using standard techniques of analysis in terms of kicks and drifts in the thin lens approximation or matrices for thick lens calculations. However these calculations are not trivial when one tries to investigate higher order effects. Numerical codes have been written ${ }^{[14]}$ that automatically generate the analytic formulation, in the form of FORTRAN routines, of higher order matrix elements. However if this calculation is possible, albeit at relatively low order (up to fifth order) it does not provide much insight on the source or the reason why some aberrations appear.

In the design of final focus systems for future linear colliders we are pushing the level of aberration correction to its limit at third order and one would like to make sure that no significant aberrations exist at higher order. In case these aberrations are indeed a problem we would like to have enough power to understand where they come from and if there is a way to correct them by either curing the cause or the effect. Also we want to make sure that tolerances are manageable. The Final Focus Test Beam is at the edge of these requirements, both in terms of aberration control and tolerances. To analyze these requirements I have studied new techniques, based on Lie algebra, developed by John Irwin at SLAC. The rest of this thesis presents these tools an the use we made of them in the development of the optics of the FFTB, mostly for the analysis of aberrations and tolerances. 


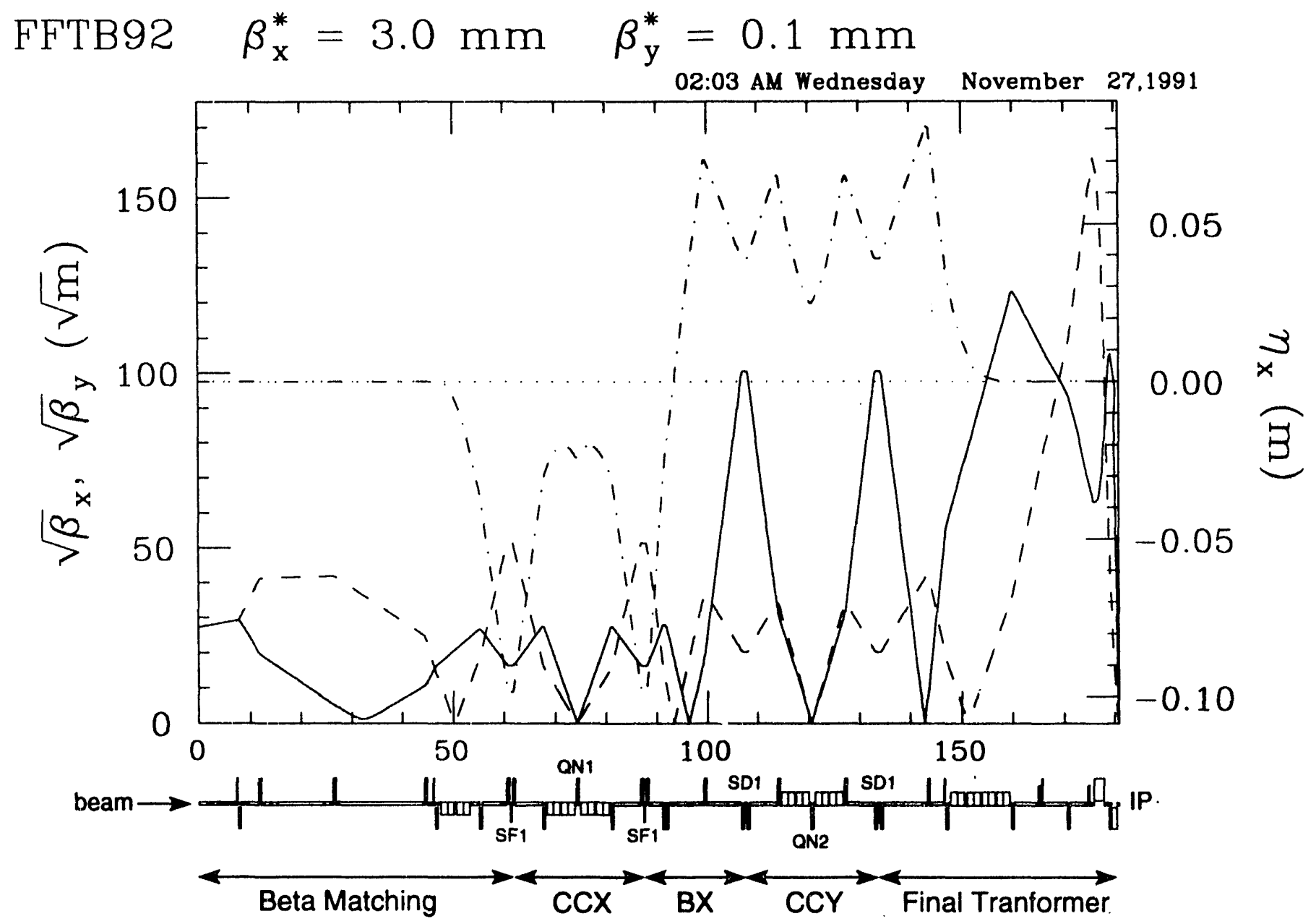

Figure 1.8. The FFTB optical functions for the nominal flat beam coufiguration. The square root of the horizontal and vertical $\beta$-functions are plotted (respectively dashes and solid) as well as the horizontal dispersion (dot-dashes). The vertical dispersion is zero everywhere. The schematic layout of the beamline is figured under the graph. This picture extends from the entrance of the FFTB (dump D10) on the left to the focal point (IP) on the right. 


\section{Lie Algebra}

\subsection{INTRODUCTION}

I give in this chapter an overview of the mathematical basis on which we will build the methous we have applied to accelerator physics. Starting form the definition of a Lie algebra, I show how this applies to Hamiltonians with the Poisson bracket as the Lie product. Lie operators and Lie transformations are then introduced with their basic properties. I then show, using two simple examples, how to actually find the equations of motion for Hamiltonian systems using Lie transformations. Finally I introduce without demonstration two theorems, the Campbell-Baker-Hausdorff theorem and the similarity transformation, which are at the center of the methods we use in the next chapter.

I have tried to keep these mathematical considerations simple and clear. There are a number of references on the mathematical theories of Lie groups and Lie algebras where all proofs and further development can be found by the interested reader. There is no need however to go beyond the concepts presented in this chapter to understand and use the methods I present in this thesis.

\subsection{DEFINITION}

I give in this section a formal definition of an algebra and the additional properties needed to constitute a Lie Algebra. These definitions are purely mathematical and quite simple. I have not attempted here to provide a comprehensive set of clefinitions. I assume that the reader is familiar with such mathematical notions as fields and vector spaces.

\subsubsection{Algebra}

A formal definition of an algebra can be given as follows:

An algebra over a field $F$ is a vector space $U$ over $F$ together with a product operation $U \times U \rightarrow U$ written $(x, y) \rightarrow x . y$, which satisfies the bilinearity properties of multiplication 
by an element of the field and the distribution (left and right) of the product with respect to the addition:

$$
\begin{array}{r}
\alpha(x . y)=(\alpha x) \cdot y=x \cdot(\alpha y) \quad \alpha \epsilon F \\
\left(x_{1}+x_{2}\right) \cdot y=x_{1} \cdot y+x_{2} \cdot y \\
\text { and } \quad x \cdot\left(y_{1}+y_{2}\right)=x \cdot y_{1}+x \cdot y_{2}
\end{array}
$$

In addition, but this is not a required property, the algebra is associative if the product satisfies

$$
x \cdot(y \cdot z)=(x \cdot y) \cdot z=x \cdot y \cdot z
$$

We will see later that this property is important for the definition of a Lie algebra.

\subsubsection{Lie Algebra}

Furthermore if the two following properties of antisymmetry and Jacoli Identity are verified for the product, the product is called a Lie product and the algebra is a Lie Algebra:

$$
\begin{gathered}
x \cdot y=-y \cdot x \\
x \cdot(y \cdot z)+y \cdot(z \cdot x)+z \cdot(x \cdot y)=0
\end{gathered}
$$

We can note here that the property of associativity of the product, not required to form an algebra, is sufficient to show that the product is not a Lic product. In other words the associativity property on the one hand and the antisymmetry and Jacobi identity on the other hand are mutually exclusive and an associative algebra cannot be a Lie algebra.

\subsubsection{Examples}

As simple examples of algebras and Lie algebras we can consider the well known set of the $n \times n$ square matrices.

We can first show very easily that this set, with the common matrix product, defines an algebra over the field of real numbers. I will not carry the demonstration ont here since it is trivial. 
However we can remark that the matrix product is not antisymmetric, namely $A . B \neq$ $-B . A$ which is one of the two properties required for a product to be a Lie product. The common matrix product is not a Lie product and the associated algebra is not a Lic algebra.

It would have been equivalent to state that because the normal matrix product is indeed associative, the algebra of the square $n \times n$ square matrices with the normal matrix product cannot be a Lie algebra

Let us now consider the commutator of two square matrices as the "product" on the same vector field, $[A, B]=A . B-B . A$ where the dot still represents the normal matrix product. It can easily be shown that this is indeed an algebra and the commutator product is not associative. The commutator product is easily shown to be antisymmetric and the Jacobi identity can be verified for all $A, B$ and $C$ in the vector ficld by a trivial expansion of $[A,[B, C]+[B,[C, A]]+[C,[A, B]]=0$. The commutator product is a Lie product and the set of the square $n \times n$ matrices with this product is a Lie algebra.

In accelerator physics this set of the square $n \times n$ matrices is well known since its introduction by $\mathrm{E}$. Courant and H. Snyder as the mathematical frame for their work on strong focusing. It is the basis of the formalism used in the computer code Transport ${ }^{[8]}$ and is the most widely used nowadays. Each element of an accelerator can be represented by a matrix mapping the coordinates of a particle (or ray) at the entrance of the element into coordinates at the exit. However the product used in this formalism is the common matrix product. An example of the Lie algebra based on the commutator in accelerator physics is the set of the symplectic matrices $\left(S P_{2 n}\right)$. For a detailed discussion on this subject, see reference 18.

\subsection{Hamiltonians and Poisson Brackets}

The notion of Hamiltonian in a dynamical system arises from the more general formulation of Lagrangian mechanics. If the equations of motion in Lagrangian formulation consist of $s$, the number of degrees of freedom, equations of second order, there are $2 s$ equations of order one to solve in the case of the Hamiltonian formulation. These equations are called canonical equations because of their simple formulation and their symmetry ${ }^{[29]}$. In general the Haniltonian of a system is a function that does not depend on time if the potential itself does not depend on time. 
The equations of motion for the canonical variables $q_{i}, p_{i}(i=1,2 \ldots, s)$ are expressed by the equations of Hamilton:

$$
\frac{d q}{d t}=\dot{q}_{i}=\frac{\partial H}{\partial p_{i}} ; \quad \frac{d p}{d t}=\dot{p}_{i}=-\frac{\partial H}{\partial q_{i}}
$$

Let $f$ be a function of the $q, p$ and $t$ variables; the total derivative of $f$ with respect to the time variable is written using the chain rule for derivation:

$$
\frac{d f}{d t}=\frac{\partial f}{\partial t}+\sum_{i} \frac{\partial f}{\partial q_{i}} \dot{q}_{i}+\frac{\partial f}{\partial p_{i}} \dot{p}_{i}
$$

The Poisson Bracket $[f, g]$ of two functions $f$ and $g$ of the variables $p_{i}, q_{i}$ is defined as

$$
[f, g]=\sum_{i} \frac{\partial f}{\partial q_{i}} \frac{\partial g}{\partial p_{i}}-\frac{\partial f}{\partial p_{i}} \frac{\partial g}{\partial q_{i}}
$$

Using the equations of Hamilton (2.6) the total derivative of $f$ with respect to time (2.7) is then rewritten

$$
\frac{d f}{d t}=\frac{\partial f}{\partial t}+[f, H]
$$

Particularly by taking $f=H$ one gets $\frac{d H}{d t}=\frac{\partial H}{\partial t}$ and if $H$ does not explicitly depend on time $\frac{d H}{d t}=0$ which expresses the conservation of some quantity $H$, usually the energy of the system.

I do not wish to elaborate more on this at this point. I will come back to this subject in the next chapter when it comes to building the Hamiltonian of optical magnetic elements for an accelerator.

\subsection{The Lie Algebras on Hamiltonians}

Consider the vector space of the differentiable functions of the generalized real variables $\{q, p\}=\left\{q_{i}, p_{i}, i=1,2, \ldots, s\right\}$ and the time $t$. The product defined as the Poisson bracket $f . g=[f, g]$, is effectively a Lie product and the algebra hereby defined is a Lie algebra.

The proof is readily obtained: From the linearity and distributivity of the derivative one infers the bilinearity and the distributivity of the Poisson bracket. 


$$
\begin{aligned}
{[\alpha f, g]=[f, \alpha g] } & =\alpha[f, g] \\
{[f+g, h] } & =[f, h]+[g, h] \\
\text { and } \quad[f, g+h] & =[f, g]+[f, h]
\end{aligned}
$$

The antisymmetry propert: results from the minus sign in the definition of the Poisson bracket:

$$
[f, g]=-[g, f]
$$

The Jacobi identity is the least obvious from the definition of the Poisson bracket but is easily developed. In terms of Poisson brackets it is written

$$
\left[f,\left[g, h_{1}\right]\right]+[g,[h, f]]+[h,[f, g]]=0
$$

This Lie algebra is the one on which we will base the rest of the work presented here.

\subsection{Lie Operators}

To every function $f(q, p, t)$ we associate a "Lie operator", $f:$, operating ou any function $g(q, p, t)$ and defined as

$$
: f: g=[f, g]
$$

In the words of Alex Dragt, "the Lie operator is a Poisson bracket waiting to happen". The powers of Lie operators are defined as

$$
\begin{aligned}
& : f:{ }^{0} g=g \\
& : f: g=[f, g] \\
& : f:{ }^{2} g=: f:(: f: g)=[f,[f, g]]
\end{aligned}
$$

The set of the Lie operators forms a vector space:

$$
a: f:+b: g:=: a f+b g: \quad \text { with a and b scalars }
$$

They act as a differentiation operator with respect to the common product and the Poisson 
bracket:

$$
\begin{gathered}
: f:(g h)=(: f: g) h+g(: f: h) \\
: f:[g, h]=[: f: g, h]+[g,: f: h]
\end{gathered}
$$

Note here that we can rewrite equation (2.9) using the antisymmetry property of the Poisson bracket and the definition of the Lip ( norator

$$
\frac{d f}{d t}=\frac{\partial f}{\partial t}-: H: f
$$

And when the function $f$ does not explicitly depend on the time variable $\left(\frac{\partial f}{\partial t}=0\right)$, which is the case for the functions we will study later in this thesis, the equation can even be restricted to the following notation

$$
\frac{d}{d t}=-: H
$$

showing that the Hamiltonian Lie operator simply consists in a time derivation.

I can now rewrite equation (2.18) for this particular case as

$$
-: H:[g, h]=\frac{d}{d t}[g, h]=\left[\frac{d g}{d t}, h\right]+\left[g, \frac{d h}{d t}\right]
$$

which forms the differentiation rule for a Poisson bracket.

Finally one can also define a product on Lie operators as the commutation between the two individual operators:

$$
\{: f:,: g:\}=: f:: g:-: g:: f:
$$

Applying this new operator to any function $h(q, p, t)$ we get

$$
\begin{aligned}
\{: f:,: g:\} \quad & =: f:: g: h-: g:: f: h=[f,[g, h]]-[g,[f, h]] \\
& =[[f, g], h]=:[f, g]: h
\end{aligned}
$$

so that we can write $\quad\{: f:,: g:\}=:[f, g]$ :

The commutator has already been shown to be a Lie product. The algebra of Lie operators with the commutator as a Lie product is a Lie algebra. Notice that this Lie algebra of Lie operators is constructed upon the Lie algebra of functions of generalized variables with the Poisson bracket as the Lie product and is therefore homomorphic to the later. 


\subsection{Lie Transformations and Properties}

\subsubsection{Definition}

Powers of Lie operators belong to the space of Lie operators. It is possible to introduce the functions that can be expanded in integral series of Lie operators and these functions also belong to the space of Lie operators. In particular the exponential function

$$
\exp (: f:)=\sum_{n=0}^{\infty} \frac{1}{n !}(: f:)^{n}
$$

is defined as the Lie transformation and its action on a function $g(q, p, t)$ is the series

$$
\exp (: f:) g=g+[f, g]+\frac{1}{2 !}[f,[f, g]]+\ldots
$$

\subsubsection{Properties}

The following properties can be verified using the series expansion of equation (2.25) and equations (2.17) and (2.18).

$$
\begin{aligned}
& e^{: f:}(g h)=\left(e^{: f:} g\right)\left(e^{: f: h}\right) \\
& e^{: f:}[g, h]=\left[e^{: f:} g, e^{: f: h !}\right. \\
& e^{: f:} g(z)=g\left(e^{: f:} z\right)
\end{aligned}
$$

The first two properties are easily shown. The last one above should be emphasized for it will be central in the treatment of optics. It can be expressed as the following rule:

"to transform a function through a Lie transformation, one needs only transform the coordinates of this function".

If we now apply the Lie transformation $e^{-\tau: H:}$ where $\mathrm{H}$ is the Hamiltonian, to the functions of canonical variables $q_{i}, p_{i}$ when these functions do not explicitly' depend on time but are taken at time $t_{0}$, we have

$$
e^{-\tau: H:} f_{t_{0}}=\left.\sum_{n=0}^{\infty} \frac{\tau^{n}}{n !} \frac{d^{n} f}{d t^{n}}\right|_{t_{0}}=f_{t_{0}+\tau}
$$

which is the usual definition of the translation of a system by a time $\tau$ using the Taylor series expansion of the function at a time $t_{0}$. 
2.0.3.1. Uniform motion

Let us assume the Hamiltonian $H=\frac{p^{2}}{2 m}$ for the movement in one climension of an object of mass $m$ with generalized coordinates $q$ and $p$. This Hamiltonian corresponds to a motion with speed $\dot{q}=\frac{\partial H}{\partial p}=\frac{p}{m}$ and the motion is uniform since $\dot{p}=\frac{\partial H}{\partial q}=0$. The solution comes then from integrating these two equations and we have, with $q_{0}$ and $p_{0}$ the initial conditions:

$$
p=p_{0} ; \quad q=q_{0}+p_{0} t
$$

If we now try to solve the same problem using Lie algebraic methods, wo have to evaluate the transformation of the coordinate function $f(q) \equiv q$ evolving according to the Hamiltonian $H$. We see here that the approach is quite different in the two methods. The classical method derives an equation of motion and therefore "maps" the input to the output coordinates, while the second method attempts to find the transformation of the function representing those coordinates. Notice here that physicists are often lax when it comes to making fine distinctions of this sort and often overlook these differences. I have found that it is however an important point here since the "mapping" approach of the problem, corresponding to the first method presented here and relying on coordinates is so obvious and natural and the manipulation of functions bearing the same name as the coordinates is often overloolied.

Following equations (2.27) and (2.25), the solution is simply the Lic transformation:

$$
e^{-t: H:} q=q-t[H, q]+\frac{t^{2}}{2}[H,[H, q]]+\ldots
$$

As one can see the operations involved here are Poisson brackets which involve only derivations. There is no integration to perform and we already can see that if a computer can be made to analytically perform a differentiation, which is one of the great results of the Differential Algebra techniques, it will be very easy to make it calculate analytically the evolution of the function q. Note that it results from the assmmptions that the Hamiltonian of a classical dynanical system can be differentiated as many times as neressany. 
The individual Poisson brackets to be calculated are:

$$
\begin{aligned}
{[H, q] } & =\left[\frac{p^{2}}{2 m}, q\right]=-\frac{p}{m} \\
{[H,[H, q]] } & =-\left[\frac{p^{2}}{2 m}, p\right]=0
\end{aligned}
$$

It is seen here that this system is actually closed and replacing the results in equation (2.29), we get, assuming that the functions at $t=0$ evaluate to $p(t=0)=p_{0}$ and $q(t=0)=q_{0}$ :

$$
q(t)=q_{0}+\frac{p_{0}}{m} t
$$

In the same way we get the evolution of the momentum function $p$ :

$$
\begin{array}{rlrl} 
& & e^{-t: H:} p & =: p-t[H, p]+\ldots \\
& \text { with } \quad[H, p] & =\left[\frac{p^{2}}{2 m}, p\right]=0 \\
& \text { giving } \quad p(t) & =p_{0}
\end{array}
$$

We have seen here how to get the same result using two different methods. In the usual method one needs to integrate the equations of Hamilton to get the result. The second one makes use of the Lie algebraic properties of the space of physical functions with the Poisson bracket as a Lie product. The evolution of any function of position and momentum is given by the Lie transformation that only requires the calculation of Poisson brackets of the Hamiltonian describing the system with these functions. Moreover according to the last property in (2.26), one needs only calculate the Poisson brackets with the functions position $q$ and momentum $p$.

I have chosen this elementary example to develop the method in detail. It may appear that the first method is simpler but the reader is reminded that in many practical cases, Hamilton's equations are not easily integrable. 
2.6.3.2. The harmonic oscillator.

I can now present a more elaborate example. The harmonic oscillator is similar to the problem of the motion in a quadrupole in accelerator physics and it involves an open system. A reduced Hamiltonian for the harmonic oscillator can be written $H=\frac{1}{2}\left(p^{2}+q^{2}\right)$.

Ones needs to calculate the different powers of the Lie operator associated with the Hamiltonian and their action on the functions position $q$ and momentum $p$ :

$$
\begin{aligned}
{[H . q]=} & : H: q=-p \quad: \quad: H:^{2} q=-: H: p=-q \quad ; \quad: H:{ }^{3} q=p \quad ; \quad: H:^{4} q=q \quad \ldots \\
& : H: p=q \quad: \quad: H:^{2} p=: H: q=-p \quad ; \quad: H:{ }^{3} p=-q \quad ; \quad: H:^{4} p=p \quad \ldots
\end{aligned}
$$

which allows us to write for the solution

$$
\begin{aligned}
e^{-t: H:} q & =q-t: H: q+\frac{t^{2}}{2}: H:^{2} q-\frac{t^{3}}{3 !}: H:^{3} q+\ldots \\
& =q+p t-q \frac{t^{2}}{2}-p \frac{t^{3}}{3 !}+\ldots \\
& =q\left(1-\frac{t^{2}}{2}+\ldots\right)+p\left(t-\frac{t^{3}}{3 !}+\ldots\right) \\
& =q \cos (t)+p \sin (t) \\
e^{-t: H:} p & =p-t: H: p+\frac{t^{2}}{2}: H:^{2} p-\frac{t^{3}}{3 !}: H:^{3} p+\ldots \\
& =p-q t-p \frac{t^{2}}{2}+q \frac{t^{3}}{3 !}+\ldots \\
& =p\left(1-\frac{t^{2}}{2}+\ldots\right)+q\left(t-\frac{t^{3}}{3 !}+\ldots\right) \\
& =p \cos (t)-q \sin (t)
\end{aligned}
$$

The last step in (2.34) and (2.35) involves the recognition of the familiar series of the sine and cosine functions.

From this second example it should now be clear how one can trat those problems in a very general way given the Haniltonian of the system. An extension of these examples to more than one dimension (two degrees of freedom) is also straightforward. 


\subsection{THEOREMS}

I present here the last two theorems needed for the rest of this work. Any Lic transformation is associated to one Hamiltonian. In the case of systems with piecewise Hamiltonians, the whole system is represented as a product of the different Lie transformations. It is often desirable to combine these into one global transformation or in other words to find the one Hamiltonian representing the whole system.

The general problem of finding the combination of two Lie trausformations is solved by the Campbell-Baker-Hausdorff theorem:

$$
\begin{gathered}
e^{: f:} e^{: g:}=e^{: h:} \\
\text { with } \quad h=f+g+\frac{1}{2}[f, g]+\frac{1}{12}[f-g,[f, g]]+\ldots
\end{gathered}
$$

There is no general expression for this series but one important aspert for computational purposes is the fact that the right hand side of equation (2.36) contains only Poisson brackets of increasing order, or derivatives of $f$ and $g$ of increasing order but no terms of order higher than one in $f$ or $g$. The demonstration of this theorem is not easy and one can refer for example to the one given by A. Dragt and J. Finn in reference 19.

The algebra of Lie transformation is non-commutative and the reordering of a product of such transformations can be performed using the similarity transformation; it is readily seen with the $\mathrm{CBH}$ theorem that the inverse of a Lie transformation $e^{: f:}$ is the Lic transformation $e^{-: f:}$ and inserting the identity $e^{: f:} e^{-: f:}$, one gets

$$
\begin{aligned}
e^{: g:} e^{: f:} & =e^{: f:}\left(e^{-: f:} e^{: g:} e^{: f:}\right) \\
& =e^{: f:} e^{: e^{-: f: g:}} \\
& =e^{: f:} e^{: g^{\prime}:}
\end{aligned}
$$

Then following the last property in $(2.26)$ we get that $g^{\prime}(z)$, with $:=\left\{q_{i}, p_{i}\right\}$, is the same function $g$ expressed now in terms of the coordinate functions transformed by $f: g^{\prime}(z)=$ $g\left(e^{: f: z)}\right.$. 
The similarity transformation can be interpreted as a simple coordinate transformation.

$$
\begin{aligned}
& q_{i} \rightarrow q_{i}+\left[f, q_{i}\right]+\frac{1}{2 !}\left[f,\left[f, q_{i}\right]\right]+\ldots \\
& p_{i} \rightarrow p_{i}+\left[f, p_{i}\right]+\frac{1}{2 !}\left[f,\left[f, p_{i}\right]\right]+\ldots
\end{aligned}
$$

If $f$ is a quadratic polynomial the change of coordinates is linear and can be represented in a matrix form. This is the Lie algebra equivalent of the familiar change of coordinates in the algebra of matrices $M^{\prime}=R M R^{-1}$ where $R^{-1}$ is the inverse of $R$.

Assuming a series of transformations, it is now possible to reorder this series by successive applications of the similarity transformation. For example considering a series of mixed linear $\left(f_{i}\right)$ and non-linear $\left(g_{i}\right)$ transformations, it is possible to move all the non-linear terms together by successively moving them through the linear terms as in the following example:

$$
\begin{aligned}
& e^{: g_{1}:} e^{: f_{1}:} e^{: g_{2}:} e^{: f_{2}:}=e^{: g_{1}:} e^{: f_{1}:} e^{: f_{2}:} e^{: g_{2}\left(e^{\left.: f_{2}: z\right):}\right.}
\end{aligned}
$$

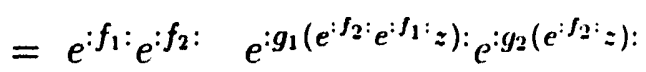

The non-linear transformations keep the same form although the coordinates on which they act are now different. Note that since the $f_{i}$ are linear transformations the familiar tools of matrix algebra can be applied here to concatenate the linear terms. The CBH theorem can be used to find a single non-linear term so that the whole series is reduced to one linear transformation times one non-linear term.

\subsection{Conclusion}

We now have at hand all the mathematical tools necessary to approach the Hamiltonian formulation of accelerator optics using Lie algebras. Let me summarize here the essential mathematical tools we will use: At the center of the theory is the notion of the Lie algebra on Hamiltonians with the Poisson bracket. The evolution of the system is given by the Lie transformation associated with the Hamiltonian of the system. Finally the CBH theorem lets us concatenate Lie transformations while the reordering of the transformations is achieved using the similarity transformation, which can also be seen as a change of variables. The separation of linear and non-linear terms is also easily achieved as shown in the last section. The similarity transformation and the $\mathrm{CBH}$ theorem are the main tools for the rest of this 
study. The mathematical basis for Lie algebra is far richer than the very quick presentation I have made here. I can only encourage the reader to further investigate this very elegant area of mathematics. 


\section{Application to Optics}

\subsection{InTRODUCTION}

I describe in this chapter the techniques used to apply the mathematical tools previously developed to the problem of magnetic optics in accelerators. After transforming the general Hamiltonian into a more specific and more manageable form I show how to separate the optics into the linear and higher-order parts using coordinate changes and the similarity transformation. The treatment of long elements and its extension to the case of beam-lines is then shown.

Two different approaches to finding higher-order effects are presented. One is a straightforward algorithm giving a good global picture of the aberrations in the beamline. The other approach is better used for the analysis of specific effects and analytical calculations. I present some examples of such analysis based on the case of the C'C'S at the FFTB. Finally I describe how to take some errors into account.

\subsection{HAMILTONIANS}

\subsubsection{The Electro-Magnetic Hamiltonian}

The general Hamiltonian of a particle of rest mass $m_{0}$, charge $r$ and canonical position and momentum $\vec{q}$ and $\vec{p}$, placed in an electromagnetic field deriving from the scalar and vector potentials $\Psi$ and $\vec{A}$ is written, with the time $t$ as the independent variable:

$$
H=\left(m_{0}^{2} c^{4}+c^{2}(\vec{p}-e \vec{A})^{2}\right)^{1 / 2}+e \Psi
$$

If the vector and scalar potentials $\vec{A}$ and $\Psi$ do not explicitly clepend on time so that $\frac{\partial H}{\partial t}=0$ the Hamiltonian is a constant of motion $\left(\frac{d H}{d t}=0\right)$ as well as an integral of motion $([H, H]=0)$. The Hamiltonian in equation $(3.1)$ is a constant and represents the total energy of the system $(H=E)$. 
It is convenient in accelerator physies to change the coordinate system and analyze the dynamics of the particles with respect to a design trajectory. The $x$ and $y$ axes lie in the plane perpendicular to the trajectory of the reference particle and the $s$ axis is along this reference trajectory. This curvilinear coordinate system has been described in many places and is very familiar to accelerator physicists.

The change of coordinates ${ }^{[6,30]}$ generates a new Haniltonian, still written $H$ however. The new constant of motion, $H=-p_{s}$, is the longitudinal momentum. Denoting by $\rho(s)$ the curvature of the design trajectory along the machine we get the following Hamiltonian:

$$
H=-e A_{s}-\left(1+\frac{x}{\rho}\right)\left[\frac{(E-e \Psi)^{2}}{c^{2}}-m_{0}^{2} c^{2}-\left(p_{x}-e A_{x}\right)^{2}-\left(p_{y}-e A_{y}\right)^{2}\right]^{\frac{1}{2}}
$$

I shall now restrict the scope of the problem to the magnetostatic case of typical accelerator magnets, where $\Psi=0$ and the vector potential $\vec{A}$ does not vary with time. It is also convenient and, because of time independence, legitimate to scale the Hamiltonian by the total momentum of the particle $p$. This requires that we scale the coordinate $p_{x}$ and $p_{y}$ giving $x^{\prime}=p_{x} / p$. the angle that the trajectory of the particle makes with the design trajectory in the horizontal direction (x plane) taken in the approximation of small angles. Sinilarly in the vertical plane $y^{\prime}=p_{y} / p$. The new Haniltonian is

$$
H=-\frac{e}{p} A_{s}-\left(1+\frac{x}{\rho}\right)\left[1-\left(x^{\prime}-\frac{e}{p} \cdot A_{x}\right)^{2}-\left(y^{\prime}-\frac{e}{p} \cdot A_{y}\right)^{2}\right]^{\frac{1}{2}}
$$

\subsubsection{Approximations}

I assume for the rest of this study that the only non-zero component of the vector potential is the longitudinal component $A_{s}$. Since $\vec{B}=\nabla \times \vec{A}$, this assumes that the longitudinal component of the magnetic ficld is zero everywhere. Although I neglect as a consequence the fringe fielris of magnetic elements, which is a good approximation in our case, the formalism is quite general. Inchuding elements with axial magnetic fields and fringe fields would not present any additional problem.

I can now expand the scpuare poot in a series since the angles d and y' are typically very small: of the order of a few tentle of a micromadian in most elements at the FFTB, and up) to a maximum of 300 to 500 mincoradia is at the focal point. 


$$
H=-\frac{e}{p} A_{s}-\left(1+\frac{x}{\rho}\right)\left[1-\frac{1}{2}\left(x^{\prime 2}+y^{\prime 2}\right)-\frac{1}{8}\left(x^{\prime 2}+y^{\prime 2}\right)^{2}+\ldots\right]
$$

Also using the same argument of small angles, we get that the effects of the fourth and higher-order terms in $x^{\prime}$ and $y^{\prime}$ in the above expansion are negligible. Note that some very general aberrations, well known to spectrometer designers, do disappear from the calculations due to this approximation; for example there are higher-order geometric aberrations appearing in quadrupoles if one keeps more terms in the development. They are due to large enough $x^{\prime}$ and $y^{\prime}$ that change the path length inside the magnet. Their effects can be calculated as perturbations and shown to be negligible for the FFTB.

All the terms we have dropped may be included in situation where they are important.

Note that the first term of the series in (3.4), a constant, does not have any effect on the dynamics of the system and can be dropped. Finally I can express the total momentum $p$ of the particle in terms of the design momentum $p_{0}$ and $\bar{\delta}=\frac{p-p_{0}}{p}$ a momentum deviation ${ }^{*}$ so that the final expression for the Hamiltonian is, given as a function of the vector potential $A_{s}$ :

$$
H=-\frac{e}{p_{0}}(1-\bar{\delta}) A_{s}-\frac{x}{\rho}+\frac{1}{2}\left(1+\frac{x}{\rho}\right)\left(x^{\prime 2}+y^{\prime 2}\right)
$$

The equations of motion can then be obtained from Hamilton's equations (2.6) and the motion, solution of these equations, can be found by integration or alternatively by applying the Lie transformation to a given coordinate function, as shown in chapter 2.

\subsubsection{Potentials}

The expression for the vector potential $A_{s}$ can be obtained from Maxwell's equations expressed in curvilinear coordinates.

* The deviation usually quoted in accelerator physics, $\delta=\frac{p-p_{0}}{p_{0}}$. Wonld lead to an infinite series of chromatic terms at all orders $1 /(1+\delta) \approx 1-\delta+\delta^{2}+\ldots$ Using $\bar{\delta}=\frac{\phi}{1+\phi}$ all chromatic effects are contained in the lincar dependence in $\bar{\delta}$. 


\subsubsection{1. dipoles}

In the case of bending magnets, horizontal here, the vector potential $A_{s}$ is the solution of a differential equation given by $B_{0}=(\nabla \times \vec{A})_{y}$ where the rotational operator is expressed in curvilinear coordinates. The solution ${ }^{[31]}$ is of the form

$$
A_{s}=\frac{B_{0} \rho}{2}\left(1+\frac{x}{\rho}\right)^{2}
$$

There are two terms of importance in this potential. The first one, $B_{0} x$, represents the main bending field and cancels the term $x / \rho$ in (3.5) so that, as was expected since we are in curvilinear coordinates, the main bending field disappears form the Hamiltonian of dipole magnets. The second term of interest is proportional to $x^{2} / \rho^{2}$ and is the expression of a weak-focusing effect in the horizontal plane.

Dipole magnets at the FFTB have typically a bending radius $\rho=725 \mathrm{~m}$ and the typical excursion from the central trajectory is a few hundreds of microns giving $x / \rho \approx 10^{-} \bar{t}$. The weak-focusing effect is therefore dropped altogether with higher-order effects in $x / \rho$. The only remaining term in the potential is the dispersion $\bar{\delta} x / \rho$ and the Haniltonian we assume for dipole magnets has the final form:

$$
H=\frac{1}{2}\left(x^{\prime 2}+y^{\prime 2}\right)-\frac{\bar{\delta} x}{\rho}
$$

\subsubsection{2. multipoles}

For multipoles, $1 / \rho=0$ and the magnetic vector potential is a solution of an equation of Laplace in two dimensions. This equation is easily obtained by writing the condition $\nabla \times \vec{B}=0$ together with $\vec{B}=\nabla \times \vec{A}$. The solution is of the general form $\operatorname{Re} \sum_{n} \frac{c_{4}}{n}(x+i y)^{n}$, with $c_{n}$ complex, containing all the normal as well as skew multipole terms. The order of the solution and the constant for a given magnet are determined by its geometry (number of poles, aperture) and the value of the field at the pole tip. By arguments of symmetry one can show that the solution of order $n$ corresponds to a magnet with $2 n$ poles and the orientation of the poles determines whether the element is "normal" or "skewed". One is a rotation of the other by an angle $\pi / 2 n$ around the longitudinal axis. 
A "normal" element is by definition one that has the horizontal midplane symmetry: A particle with a trajectory starting in the horizontal plane remains in the horizontal plane when passing through this element. The consequence on the symmetry of the transverse components of the field is

$$
\begin{aligned}
& B_{x}(x,-y, s)=-B_{x}(x, y, s) \\
& B_{y}(x,-y, s)=B_{y}(x, y, s)
\end{aligned}
$$

The strength of the multipole is given by the boundary condition at $\sqrt{x^{2}+y^{2}}=a$ the radius of the aperture of the magnet where the field is equal to the pole tip magnetic field $B_{0}$. The solutions at order $n$ for the normal and skew multipole elements respectively are written as the real and imaginary part of the general solution above:

$$
\begin{aligned}
& -\frac{e}{p_{0}} A_{s}=\frac{K_{n}^{\prime}}{n !} \operatorname{Re}(x+i y)^{n} \\
& -\frac{e}{p_{0}} A_{s}=\frac{K_{n}^{-1}}{n !} \operatorname{Im}(x+i y)^{n}
\end{aligned}
$$

The absolute values of the strengths are defined, with the magnetic rigidity $(B \rho)=\frac{p_{0}}{e}$, as

$$
\left|K_{n}\right|=\left|K_{n}^{\prime}\right|=(n-1) ! \frac{B_{0}}{a^{n-1}(B \rho)}
$$

For example the following Hamiltonians determine the dynamics of a particle in respectively a drift space (no field), a quadrupole, a skew quadrupole and a sextupole:

$$
\begin{aligned}
\text { drift space: } & H=\frac{1}{2}\left(x^{2}+y^{\prime 2}\right) \\
\text { quadrupole: } & H=\frac{1}{2}\left(x^{2}+y^{\prime 2}\right)+\frac{1}{2} K_{2}(1-\bar{\delta})\left(x^{2}-y^{2}\right) \\
\text { skew quadrupole: } & H=\frac{1}{2}\left(x^{2}+y^{\prime 2}\right)+K_{2}^{\prime \prime}(1-\bar{\delta}) \cdot x y \\
\text { sextupole: } & H=\frac{1}{2}\left(x^{\prime 2}+y^{\prime 2}\right)+\frac{1}{3 !} K_{3}(1-\bar{\delta})\left(x^{3}-3 \cdot y^{2}\right)
\end{aligned}
$$




\subsubsection{3. summary}

The following table lists the potential part of all normal and skew multipoles at order up to five. I define the potential part of a Hamiltonian as the part deriving from a potential, in our case $-\frac{e}{p_{0}} A_{s}(1-\bar{\delta})$, while the kinematic part exists even in the absence of a potential. The Hamiltonian of a drift space contains only a kinematic part.

\begin{tabular}{|c|c|c|}
\hline element & normal & skew \\
\hline dipole & $\bar{\delta} x / \rho$ & $\bar{\delta} y / \rho$ \\
\hline quadrupole & $\because \frac{x_{2}}{2}(1-\bar{\delta})\left(x^{2}-y^{2}\right)$ & $K_{2}^{\prime \prime}(1-\bar{\delta}) x y$ \\
\hline sextupole & $\frac{\kappa_{3}}{3 !}(1-\bar{\delta})\left(x^{3}-3 x y^{2}\right)$ & $\frac{K_{3}^{\prime}}{3 !}(1-\bar{\delta})\left(3 x^{2} y-y^{3}\right)$ \\
\hline octupole & $\frac{\Pi_{4}}{4 !}(1-\bar{\delta})\left(x^{4}-6 x^{2} y^{2}+y^{4}\right)$ & $\frac{\bar{n}_{1}^{\prime}}{4 !}(1-\bar{\delta})\left(4 x^{3} y-4 x y^{3}\right)$ \\
\hline decapole & $\frac{K_{5}}{\overline{5} !}(1-\bar{\delta})\left(x^{5}-10 x^{3} y^{2}+5 x y^{4}\right)$ & $\frac{K_{s}^{\prime \prime}}{5 !}(1-\bar{\delta})\left(5 x^{4} y-10 x^{2} y^{3}+y^{5}\right)$ \\
\hline
\end{tabular}

Table 3.1. The potential parts, normal and skew, of the Haniltonians for te first few orders. Note that for dipoles and since we are in curvilinear coordinates, only the dispersive part appears here and no order one effects are present.

The Hamiltonians defined contain all the dynamics of the system, considering of course the approximations made in the preceding section, and one can now treat a multipole element in the same way I have treated the two simple examples in the previous chapter. Note that the uniform motion example is to be compared to that of a chift here and the example of the harmonic oscillator is analogous to the case of the quadrupole.

Notice here that no Hamiltonians of order one appear in the above table since the main bending field disappears by virtue of the curvilinear coordinates. Hamiltonians of order one will however appear when considering dipole correctors, which are not a part of the design lattice, or errors or misplaced elements.

\subsection{PRINCIPLES}

\subsubsection{Thin elements}

The Lie transformation associated with the Hamiltonian as defined above is written, for a small slice of length $d s, \exp (-d s: H:) . \quad H$ is in general composed of a linear part 
$H_{l}$ of order two in the generalized coordinates and $\bar{\delta}$, and a non-linear part $H_{n l}$ of order three and higher. It is possible, in the thin-lens approximation $d s \rightarrow 0$, to rewrite the Lie transformation associated with the slice, as the product

$$
\exp (-d s: H:)=\exp \left(-d s: H_{l}:\right) \exp \left(-d s: H_{n l}:\right)
$$

The linear part $H_{l}$ contains the kinematic part of the Hamiltonian as well as the potential part of order two if present. This is equivalent to neglecting all the terms in $d s^{n}, n \geq 1$ in the $\mathrm{CBH}$ expansion since $H_{l}$ and $H_{n l}$ do not commute.

\subsubsection{Linear Transformations}

A Lie transformation of the form $M=\exp \left(-l: H_{l}:\right)$ where $H_{l}$ is of order two is a linear transformation that can be represented by a linear map. Following section (2.7) this map can be represented in a matrix form $[M]$. Although maps are used for the transformation of functions, the matrix form of a linear map is obviously equal to the familiar linear $R$ matrix representing the linear optics in the Transport formalism.

$$
[M]=R
$$

The tools and methods developed for linear optics in any matrix manipulation codes such as Transport ${ }^{[8]}$ can then be used to treat the linear optics in this Lie algebra framework.

\subsubsection{Outline of the Methods}

I have now shown how one can separate the linear optics from the non-linear terms in the Hamiltonians developed above. The linear optics is simply treated using the well-known Transport matrix theory. The rest of the analysis will consist in taking a series of thin-lens transformations and first assemble them into a thick element. Similarly taking a series of thick elements, the beamline itself can be assembled. 


\subsection{Thick Elements}

\subsubsection{Method}

Having defined the thin-lens expression of the Lie transformations, I can now concatenate the thin slices, taking the product of the transformations, to form a thick element. I choose to represent this thick element as the combination of two linear maps $M_{l / 2}$ and a single nonlinear transformation at the center:

$$
\prod_{i=1}^{n} M_{d s_{i}} e^{-d s_{i}: H\left(s_{i}\right):}=M_{l / 2} e^{-l: H_{i}:} M_{l / 2}
$$

The linear transformation $M_{d s_{i}}$ is the same for all the slices while each slice at location $s_{i}$ has a nonlinear Hamiltonians $H\left(\left(x\left(s_{i}\right), y\left(s_{i}\right)\right)\right.$. By successive application of the similarity transformation, following the rules defined in the previous chapter, I transform all the nonlinear kicks of individual slices to the center of the element:

$$
M_{l / 2} e^{-l: H_{t}:} M_{l / 2}=(\underbrace{M M \ldots M}_{n / 2}) \prod_{i=1}^{n} e^{-d s_{1}: H_{u l}^{\prime}(i):}(\underbrace{M I M \ldots M}_{n / 2})
$$

The linear $M_{l / 2}$ is obviously equal to the product of the linear transformation of half of the total number of slices, corresponding to the linear map across half the length of the thick element. And following the rule for transforming the nonlinear terms, the $H_{n l}^{\prime}(i)$ are expressed at the center of the element by simply transforming the coordinates in the $H\left(x\left(s_{i}\right), y\left(s_{i}\right)\right)$. i.e. writing $r\left(s_{i}\right)$ and $y\left(s_{i}\right)$ as a function of the coordinates at the center.

The last step is to obtain a single non-linear Hamiltonian $H_{t}$ using the C.BH theorem:

$$
l H_{t}=\sum_{i}^{n} H_{n l}^{\prime}(i) d s_{i}+\frac{1}{2} \sum_{i=1}^{n} \sum_{j=i}^{n}\left[H_{n l}^{\prime}(i), H_{n l}^{\prime}(j)\right] d s_{i} d s_{j}+\ldots
$$




\subsubsection{First order in $\mathrm{CBH}$}

The first term in the above expansion is simply the kick of the elementary slices summed over all the slices or the main effect of the multipole, now expressed at the center of the element. In the limit $d s \rightarrow 0$ the summation becomes an integral over the length of the magnet:

$$
\sum_{i}^{n} H_{n l}^{\prime}(i) d s_{i}=\sum_{i}^{n} H_{n l}\left(x\left(s_{i}\right), y\left(s_{i}\right)\right) d s_{i} \rightarrow \int_{-L / 2}^{L / 2} H_{n l}(x(s), y(s)) d s
$$

where $H(s)$ is a function of the elementary coordinate functions $r(s)$ and $y(s)$. The evolution of the elementary coordinate functions along the magnet is supposed known from the linear matrix theory.

\subsubsection{1. multipoles}

For multipoles of order three and higher, i.e. without a quadrupole part, the linear transport is that of a drift space; $x(s)=x_{c}+s x_{c}^{\prime} ; y(s)=y_{c}+s y_{c}^{\prime}$.

Taking the sextupole as an example the integral yields

$$
H=\frac{K_{3}}{3 !} L\left(x_{c}^{3}-3 x_{c} y_{c}^{2}\right)+\frac{K_{3}}{24} L^{3}\left(x_{c} x_{c}^{\prime 2}-2 x_{c}^{\prime} y_{c} y_{c}^{\prime}-x_{c} y_{c}^{\prime 2}\right)
$$

The first term is the main sextupole effect for the magnet. The second term is a higherorder effect in the length of the magnet and is typically smaller than the first one by a factor $(L / 3)^{2}$. At the FFTB this factor is at least equal to $10^{-6}$. The second term can be neglected in our case.

\subsubsection{2. quadrupoles}

For quadrupoles, and all elements with a quadrupole component of strength $K$, the linear transport is given, with $\phi(s)=\sqrt{\left|K^{\prime}\right|} s$ and for $K^{\prime}>0$, by

$$
\begin{aligned}
& x(s)=\cos \phi(s) x_{c}+\frac{1}{\sqrt{\left|\Lambda^{\prime}\right|}} \sin \phi(s) x_{c}^{\prime} \\
& y(s)=\cosh \phi(s) y_{c}+\frac{1}{\sqrt{\left|\Lambda^{\prime}\right|}} \sinh \phi(s) y_{c}^{\prime}
\end{aligned}
$$


The rete of $x$ and $y$ are exchanged for $K<0$. For a pure quadrupole the integral yields:

$$
\begin{aligned}
H=\frac{1}{2} K L \bar{\delta} & \mid l_{c}^{2} \frac{1}{2}\left(1+\frac{\sin \phi}{\phi}\right)+x_{c}^{\prime 2} \frac{1}{2\left|K^{\prime}\right|}\left(1-\frac{\sin \phi}{\phi}\right) \\
& \left.-y_{c}^{2} \frac{1}{2}\left(1+\frac{\sinh \phi}{\phi}\right)-y_{c}^{\prime 2} \frac{1}{2|K|}\left(1-\frac{\sinh \phi}{\phi}\right)\right]
\end{aligned}
$$

which can be rewritten

$$
H=\frac{1}{2} K L \bar{\delta}\left[a_{x} x_{c}^{2}+\frac{1}{|K|} b_{x} x_{c}^{\prime 2}-a_{y} y_{c}^{2}-\frac{1}{|K|} b_{y} y_{c}^{\prime 2}\right]
$$

For $K^{-}<0$, one needs only exchange $a_{x}$ with $a_{y}$ as well as $b_{x}$ with $b_{y}$.

The $a_{x}, b_{x}, a_{y}, b_{y}$ are corrections to the simple minded Hamiltonian for the guadrupole. For most quadrupoles at the FFTB the phase $\phi$ is of the order of 0.3 ladians and the following approximations are reasonably good: $a_{x} \approx 1, a_{y} \approx 1$ and $b_{x} \approx \frac{\Phi^{2}}{12}, b_{y} \approx \frac{\Phi^{2}}{12}$. The final quadrupoles are much stronger and have a phase of the order of onc radian. The following table lists the FFTB magnets by type and shows the values of the phase and correction factors. Note that the final quadrupoles (QC2, QX1, QC1) are sufficiently strong that there is a correction of about $10 \%$ to the thin-lens approximation chromaticity calculation. Since the final quadrupoles also produce most of the chromaticity of the line, one must take these corrections into account.

\subsection{3. second-order in C'BH}

The second term in the expansion of the $\mathrm{CBH}$ theorem represents the interaction of the main multipole term with itself; the effect of the kick produced by a given slice on the kicks produced by the subsequent slices. It can also be replaced by integrals over the length of the magnet:

$$
\begin{aligned}
& \frac{1}{2} \sum_{i=1}^{n} \sum_{j=1}^{n}\left[H_{n l}^{\prime}(i), H_{n l}^{\prime}(j)\right] d s_{i} d s_{j} \\
& \\
&=\frac{1}{2} \sum_{i=1}^{n} \sum_{j=i}^{n} d s_{i} d s_{j}\left[H_{n l}^{\prime}\left(x\left(s_{i}\right), y\left(s_{i}\right)\right), H_{u l}^{\prime}\left(x\left(s_{j}\right), y\left(s_{j}\right)\right)\right] \\
& \rightarrow \frac{1}{2} \int_{-L / 2}^{L / 2} d s \int_{s}^{L / 2} d s^{\prime}\left[H(x(s), y(s)), H\left(. x^{\prime}\left(s^{\prime}\right), y\left(s^{\prime}\right)\right)\right]
\end{aligned}
$$

Note that the Poisson bracket can be developed and expressod in tormo of flue olementaly 


\begin{tabular}{|c|c|c|c|c|c|}
\hline quad. type & $\phi$ (rad.) & $a_{x}$ & $b_{x}$ & $a_{y}$ & $b_{y}$ \\
\hline Q5 & 0.2058 & 1.0035 & -0.0035 & 0.9965 & 0.0035 \\
Q6 & 0.1816 & 0.9973 & 0.0027 & 1.0028 & -0.0028 \\
Q.40 & 0.1050 & 0.9991 & 0.0009 & 1.0009 & -0.0009 \\
Q.A1 & 0.2826 & 0.9934 & 0.0066 & 1.0067 & -0.0067 \\
Q.A2 & 0.2111 & 1.0037 & -0.0037 & 0.9963 & 0.0037 \\
QN1 & 0.3181 & 0.9916 & 0.0084 & 1.0085 & -0.0085 \\
QN2 & 0.3216 & 1.0087 & -0.0087 & 0.9914 & 0.0086 \\
QN3 & 0.2338 & 0.9955 & 0.0045 & 1.0046 & -0.0046 \\
QM1 & 0.3216 & 0.9914 & 0.0086 & 1.0087 & -0.0087 \\
Q.12 & 0.3181 & 1.0085 & -0.0085 & 0.9916 & 0.0084 \\
QM3 & 0.2338 & 1.0046 & -0.0046 & 0.9955 & 0.0045 \\
QT1 & 0.3137 & 0.9918 & 0.0082 & 1.0082 & -0.0082 \\
QT2 & 0.2871 & 1.0069 & -0.0069 & 0.9932 & 0.0068 \\
QT3 & 0.3292 & 0.9910 & 0.0090 & 1.0691 & -0.0091 \\
QT4 & 0.2231 & 1.0042 & -0.0042 & 0.9959 & 0.0041 \\
QC5 & 0.2643 & 1.0058 & -0.0058 & 0.9942 & 0.0058 \\
QC4 & 0.1727 & 1.0025 & -0.0025 & 0.9975 & 0.0025 \\
QC3 & 0.1279 & 1.0014 & -0.0014 & 0.9986 & 0.0014 \\
\hline QC2 & 0.9509 & 0.9280 & 0.0720 & 1.0788 & -0.0788 \\
QN1 & 0.2820 & 1.0067 & -0.0067 & 0.9934 & 0.0066 \\
QC1 & 1.1535 & 1.1185 & -0.1185 & 0.8963 & 0.1037 \\
\hline
\end{tabular}

Table 3.2. Correction factors to the first order Hamiltonians for the FFTB quadrupoles. The factors are defined in the text and are dimensionless.

$\left[x(s), x\left(s^{\prime}\right)\right]$ and $\left[y(s), y\left(s^{\prime}\right)\right]$. In the case of multipoles of order three and higher for which the linear transport is that of a drift, they are

$$
\left[r(s), r\left(s^{\prime}\right)\right]=\left[y(s), y\left(s^{\prime}\right)\right]=s^{\prime}-s
$$

The integral is then easily calculated and gives the long clement effect in terms of the 
coordinates at the center of the magnet.

Note that since this long-element Hamiltonian involves the calculation of a Poisson brackeí, its order is $(2 n-2)$ if $n$ is the original multipole order. The long sextupole term $H_{l s}=\frac{\kappa^{2} L^{3}}{48}\left(x_{c}^{2}+y_{c}^{2}\right)^{2}$ is of order four (octupole like) and its calculation is given in detail in Appendix B.

In the case of the quadrupole and with $\Delta \phi=\sqrt{\left|{ }^{\prime}\right|}\left(s^{\prime}-s\right), \Lambda^{\prime}>0$, the elementary Poisson brackets are written,

$$
\begin{aligned}
{\left[x(s), x^{\prime}(s)\right] } & =\frac{1}{\sqrt{\left|K^{\prime}\right|}} \sin \Delta \phi \\
\text { and } \quad\left[y(s), y^{\prime}(s)\right] & =\frac{1}{\sqrt{|\kappa|}} \sinh \Delta \phi .
\end{aligned}
$$

The result of the double integral gives the so called second-order chromaticity, taking $H(s)=$ $\frac{\kappa_{2}}{2} \bar{\delta}\left(x(s)^{2}-y(s)^{2}\right)$ and $\phi=\sqrt{|K|} L:$

$$
\begin{gathered}
\frac{1}{2} \int_{-L / 2}^{L / 2} d s \int_{s}^{L / 2} d s^{\prime}\left[\frac{K_{2}}{2} \bar{\delta}\left(x(s)^{2}-y(s)^{2}\right), \frac{K_{2}}{2} \bar{\delta}\left(x\left(s^{\prime}\right)^{2}-y\left(s^{\prime}\right)^{2}\right)\right] \\
=\frac{K_{2}^{2}}{2} \bar{\delta}^{2} \int_{-L / 2}^{L / 2} d s \int_{s}^{L / 2}\left\{x(s) x\left(s^{\prime}\right)\left[x(s), x\left(s^{\prime}\right)\right]+y(s) y\left(s^{\prime}\right)\left[y(s), y\left(s^{\prime}\right)\right]\right\} \\
=\frac{K_{2} L}{2} \bar{\delta}^{2}\left\{\begin{array}{c}
x_{c}^{2} \frac{1}{8}\left(1-2 \cos \phi+2 \frac{\sin \phi}{\phi}-\frac{\sin 2 \phi}{2 \phi}\right) \\
+x_{c}^{\prime 2} \frac{1}{8}\left(1+2 \cos \phi-2 \frac{\sin \phi}{\phi}-\frac{\sin 2 \phi}{2 \phi}\right) \\
+y_{c}^{2} \frac{1}{8}\left(-1+2 \cosh \phi-2 \frac{\sinh \phi}{\phi}-\frac{\sinh 2 \phi}{2 \phi}\right) \\
+y_{c}^{\prime 2} \frac{1}{8}\left(1+2 \cos \phi-2 \frac{\sinh \phi}{\phi}-\frac{\sinh 2 \phi}{2 \phi}\right)
\end{array}\right\}
\end{gathered}
$$

and assuming $K>0$. This can be rewritten

$$
\frac{K_{2} L}{2} \bar{\delta}^{2}\left\{x_{c}^{2} c_{x}+x_{c}^{\prime 2} d_{x}+y_{c}^{2} c_{y}+y_{c}^{\prime 2} d_{y}\right\}
$$

The coefficients $c_{x}, d_{x}, c_{y}$ and $d_{y}$ are those of the second-order chromaticity terms in quadrupoles. For $K<0$, one needs only exchange $c_{x}$ with $c_{y}$ as well as $d_{x}$ with $d_{y}$. The coefficients 
are approximated for small $\phi$ by $c_{x} \sim \phi^{2} / 6, d_{x} \sim-\phi^{+} / 5$ !, $c_{y} \sim \phi^{2} / 6$ and $d_{y} \sim-\phi^{4} / 5$ !. The effect is very smail for most quadrupoles at the FFTB.

Note that the CBH expansion (3.16) contains terms that involve more Poisson brackets which are therefore of even higher order. They are usually negligible but are accessible through a simple extension of the formalism.

\subsection{Beam Line Analysis}

\subsubsection{First approach}

The same methods using the similarity transiormation, as a change of coordinates, and the CBH theorem can be used to treat a beamline. Starting from the concatenation of all the thick elements in the form of the product of alternatively linear and higher-order transformations, the similarity transformation is applied successively to change the coordinates of all nonlinear terms and express them at a single common location, usually the end of the line (IP). The total Hamiltonian $H_{t}$ for the complete beamline is given by

$$
\begin{aligned}
e^{: H_{t}:} & =\left(M_{1} e^{: H_{1}:} M_{1}\right) \ldots\left(M_{n-1} e^{: H_{n-1}:} M_{n-1}\right)\left(M_{n} e^{: H_{n}:} M_{n}\right) \\
& =\left(M_{1} e^{: H_{1}:} M_{1}\right) \ldots\left(M_{n-1} e^{: H_{n-1}:} M_{n-1} M_{n} M_{n}\right) e^{: H_{n}^{\prime}:} \\
& =\left(M_{1} e^{: H_{1}:} M_{1}\right) \ldots M_{n-1} M_{n-1} M_{n} M_{n} e^{: H_{n-1}^{\prime}: e^{: H_{n}^{\prime}:}} \\
& =\tilde{M} \prod_{i=1}^{n} e^{: H_{i}^{\prime}:}
\end{aligned}
$$

with $\tilde{M}=M_{1} M_{1} \ldots M_{n} M_{n}=\prod_{i=1}^{n} M_{i}^{2}$ is the linear transformation for the entire line. It is known from the linear design and is related, as seen above, to the usilal linear $R$ matrices of the Transport formalism. The Hamiltonian $H_{i}^{\prime}$ is the Hamiltonian of element $i$ transformed through $M_{i} \prod_{j=i+1}^{n} M_{j}^{2}$, hence $H_{i}^{\prime}$ is now expressed in terms of IP coordinates.

The second part contains the product of all the nonlinear transformations now collated in one single location, the IP. The nonlinear optics of the entire line is expressed in terms of one set of local coordinate functions. Successive applications of the CBH theorem to this product of Lie transformations yields a single final Haniltonian containing the information on the nonlinear behavior of the whole line. The calculations are straightforward but tedious after a comple of elements. The FFTB has over thirty different elements to take into account. 
This systemat ie method is one that can be antomated easily and yields the total nomlincal Haniltonian of the line giving a picture of the aberration content of the optics. While it is difficult with traditional matrix-based optics methods to get terms at third ${ }^{(11)}$ order and quasi impossible to get higher-order ${ }^{[14]}$ terms, the method I described can give results at practically any order although I will show later that it is not necessary to go beyond fifth order in the case of the FFTB. The maximum order is limited only by how far one carries the CBH expansion, provided the long elements effects have been taken into account correctly.

These methods may then seem like the panacea for getting knowleclge on the nonlinear optics of accelerators. However since we are trying to carefully cancel harmful aberrations, one is also interested in studying these cancellations in detail as well as looking at how optical elements interact with each other. It might be possible to design better optics using this information. To this end I need to tell exactly which aberration is coming from which clement or which combination of elements. Some fifth order aberrations that exist at the FFTB would certainly be detected with this method but the mechanism of their appearance, in short the interaction of the central quadrupole in the C CY, with the sextupoles around it, would be difficult to guess and possible correction nearly inpossible to invent with this method alone. Oide ${ }^{[28)}$ has identified these aberrations using a nethod based on kicks on individual trajectories. I show in the next section how to easily calculate them using Lie algebra techniques.

\subsubsection{Looking for insight}

There is a more powerful way to use these Lie algebra techuiques, a methodology that gives a lot more insight in the optics than the one I have described in the previons paragraph. Note that the two methods are certainly not exclusive but are nore complementary: the first one might detect a significant aberration in the total nomlinear Haniltonian, the second method will help in the careful, insightful study of it.

Let me recall here that the optics of final focus systems is based on the cancellation by sextupoles of the chromaticity introduced by mostly the final cuadrupoles. There are five large chromatic sources: four sextupoles and the final quadrupoles. The sextupoles are also sources of strong geometric aberations.

In the method outlined above these strong Hamiltonians are mixed with weaker ones, like the chromaticity of other quadrupoles or the long-sextupole aberration. The approach 
here is to select only the nonlinearities I am interested in and consider the rest of the optics as linear. I therefore treat only a chosen subset of the problem, allowing to treat one effect at a time.

After that the analysis could follow exactly the approach presented in the previous section: individual non-linear Hamiltonians are transformed (similarity transformations) to a common reference point (e.g. the IP) where they are combined using the CBH theorem. Since we are looking at only a few terms, this can be done analytically and the interplay of these terms is easily seen. There are other ways to do this refined analysis and as an example I use, in the next two sections, the particular symmetry of the CCS at the FFTB.

The Chromatic Correction Section indeed forms a self consistent section inside the final focus. Its purpose being to ideally introduce a single term, the chromaticity needed to cancel that of the final quadrupoles. The idea here is to treat the CCS as a beam line by itself, reducing it to the now familiar form of a linear transformation $(-I)$ and a global Hamiltonian containing all of its nonlinear terms. The entire line can then be built from those reduced sections and other individual elements.

\subsubsection{Geometrics cancellation in the CCS}

The Chromatic Correction Sections are at first order $-I$ sections. That is they are transparent for the linear optics. I consider now the C'S as a small beam line starting at the first sextupole and ending at the second sextupole. The separation of the linear transformation and the higher-order Hamiltonians is made using tle second sextupole as the reference location where all Hamiltonians will be transformed.

I choose to treat only the case of sextupolar abcrations in this section. All other elements within the C'CS are considered linear, including the quadrupoles whose chromaticity is here neglected. The Hamiltonian of a sextupole is, in the presence of horizontal dispersion $\eta$ and expressed in local coordinates:

$$
\begin{aligned}
H_{s} & =\frac{k_{s}}{3 !}\left((x+\eta \bar{\delta})^{3}-3(x+\eta \bar{\delta}) y^{2}\right) \\
& =\frac{k_{s}}{3 !}\left(x^{3}-3 x y^{2}\right)+\frac{k_{s}}{2} \eta \bar{\delta}\left(x^{2}-y^{2}\right)+\frac{k_{s}}{2} \eta^{2} \bar{\delta}^{2} x+\frac{k_{s}}{3 !} \eta^{3} \bar{\delta}^{3}
\end{aligned}
$$

The second line in (3.28) shows the main geometric $\left(H_{g}=\frac{k}{3}{ }^{4}\left(x^{3}-3 x y^{2}\right)\right)$ and the main chromatic $\left(H_{c}=\frac{k_{s}}{2} \eta \bar{\delta}\left(x^{2}-y^{2}\right)\right)$ aberrations introduced by the sextupole. I further limit this 
study to these two terms. The two remaining terms in (3.28) are the second-order clispersion and a purely chromatic term with no effect.

I can now represent the CCS, from sextupole center to sextupole center by the following sequence of transformations. Note that in local coordinates the aberrations of a sextupole commute and I can reorder them. The goal of this transformation being of course to separate the linear optics $(-I)$ from the nonlinear terms:

$$
\begin{aligned}
e^{: H_{c c s}:} & =e^{: H_{c}:} e^{: H_{g}:}(-I) e^{: H_{g}:} e^{: H_{\mathrm{c}}:} \\
& =(-I) e^{: H_{c}:}\left(e^{:-H_{g}:} e^{: H_{g}:}\right) e^{: H_{c}:} \\
& =(-I) e^{: 2 H_{c}:}
\end{aligned}
$$

Obviously the $(-I)$ transforms $H_{c}$ into $H_{c}$ since it is an even term in $x$ and $y$ and the dispersion is the same at both sextupoles, while $H_{g}$ goes into $-H_{g}$ since it is an odd term in $x$ and $y$. The geometric aberrations simply vanish at the second sextupole and one is left with the sum of the chromaticities introduced by the sextupoles, since they obviously commute. This is a well known result, the basis of chomaticity correction for final focus systems.

This result has an important consequence for the analysis of the rest of the line. The geometric aberrations having vanished locally there is no need to talie them into account for the building of the Hamiltonian of the whole line. There cannot be any direct effect of the interaction between the sextupole geometric aberrations and the chromaticity of the final quadrupoles for example.

\subsubsection{Fifth order aberrations}

Other terms arise from the C'CS if one considers a more extended model of it. Oide ${ }^{[28]}$ has shown that some fifth order aberrations originate in the "chromatic breakdown" of the $-I$ : Due to the chromaticity of the quadrupoles inside the C'CS, the $-I$ is only exact for onmomentum particles. The linear optics is slightly different for off-energy particles. Therefore the chromatic and geometric aberrations they experience is different from what I have shown in the previous section: The net chromatic kick is not exactly twice that of a single sextupole and the geometric aberrations are not strictly cancelled, resulting in fifth order aloemations. 


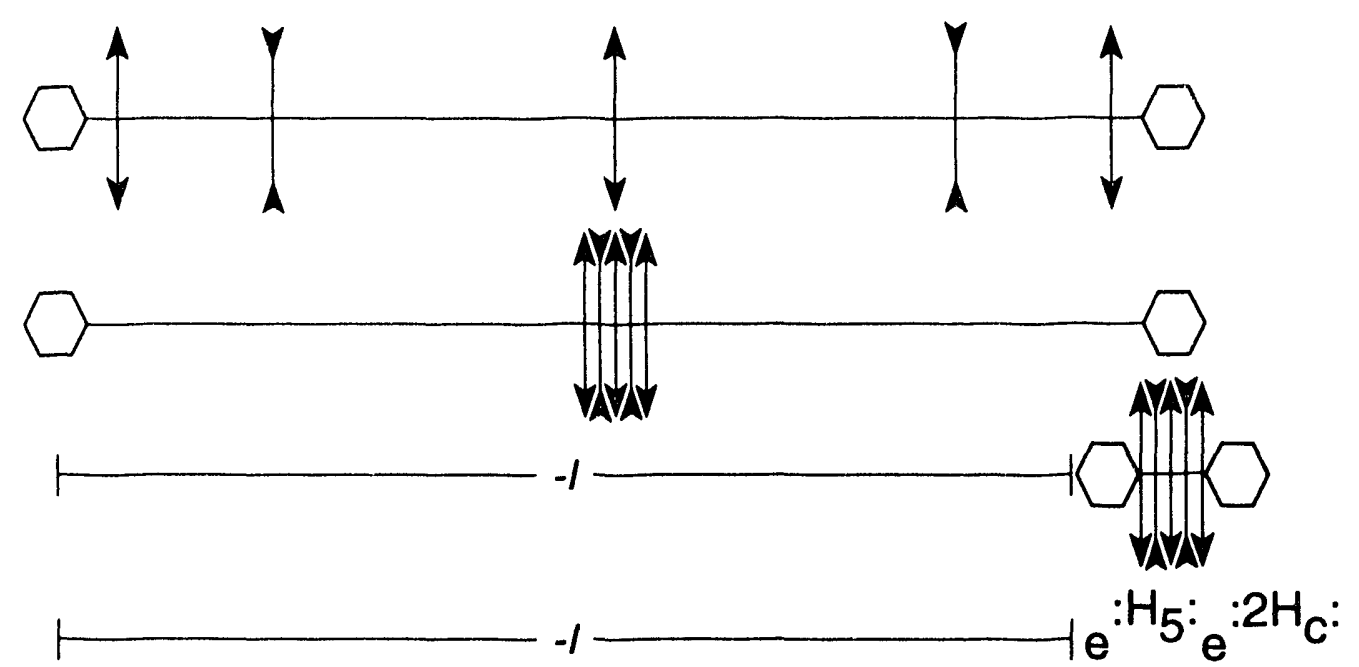

Figure 3.1. The model and the method used for the study of the CCS. After linear transport, shown in steps here, of all the elements into one location, the linear part of the line, $-I$, is virtually extracted. One is left with the non-linear kicks. The sextupole geometric kicks cancel and one is finally left with the chromaticity correction term, $2 H_{c}$, and a fifth order residual and inherent aberration.

The model I choose to treat here is that of the full CCS at the FFTB: Two sextupoles separated by a $-I$ transformation made of five quadrupoles.

The first step is to combine the Hamiltonians representing the chromaticity for all five quadrupoles into a single Hamiltonian ( $c f$. second line in above figure). The reference location I choose is the quadrupole at the center of the CCS. It is located exactly $\pi / 2$ from either sextupole. The transformation is linear and is equivalent to a change of coordinates in the individual Hamiltonians. Using the $\mathrm{CBH}$ theorem one builds the single Hamiltonian $H_{q}$ representing the total chromaticity of all the quadrupoles of the C'CS. Expressed at the center cularlrupole, it is of the general form

$$
H_{q}=\bar{\delta}\left(a x^{2}+b x^{2}+c y^{2}+d y^{\prime 2}\right)
$$

where $a, b, c$ and $d$ are coefficients functions of the linear lattice in the CCS. Note that the end quadrupoles, closest to the sextupoles, contribute mostly to the $b$ and $d$ coefficients while the center quadrupoles contribute essentially to $a$ and $c$.

Note that no term proportional to $x x^{\prime}$ or $y y^{\prime}$ appear in this formula. Since the section is symmetric the Hamiltonian is the same irrespective of the direction we use for the calculation. 
This is equivalent to applying a time reversal for which $x^{\prime}$ is changed to $-x^{\prime}$ and $y^{\prime}$ to $-y^{\prime}$. No term of odd order in $x^{\prime}$ or $y^{\prime}$ can therefore appear.

The second step of this argument is very similar to the treatment of the geometrics of the CCS in the previous section: After linear transformation of the three "elements" to the center of the second sextupole of the pair ( $H_{q}^{\prime}$ is the transformed of $H_{q}$ ), the situation is represented by the following product ( $c f$. third line in above figure):

$$
e^{: H_{c c s}:}=(-I) e^{: H_{c}:}\left(e^{:-H_{g}:} e^{: H_{q}^{\prime}:} e^{: H_{y}:}\right) e^{: H_{r}:}
$$

The first similarity transformation gives

$$
e^{: H_{c c s}:}=(-I) e^{: H_{c}:} e^{: H_{q}^{\prime \prime}:} e^{: H_{c}:}
$$

where

$$
\begin{aligned}
H_{q}^{\prime \prime} & =e^{-: H_{g}:} H_{q}^{\prime} \\
& =H_{q}^{\prime}\left(e^{-: H_{g}:} x, \ldots, e^{-: H_{g}:} y^{\prime}\right) \\
& =H_{q}^{\prime}\left(x, x^{\prime}-\frac{\partial H_{g}}{\partial x}, y, y^{\prime}-\frac{\partial H_{g}}{\partial y}\right)
\end{aligned}
$$

After insertion of an identity, a second similarity transformation yiclds

$$
e^{: H_{c c s}:}=(-I) e^{: H_{q}^{\prime \prime \prime}:} e^{2: H_{c}:}
$$

where now

$$
\begin{aligned}
H_{q}^{\prime \prime \prime} & =e^{: H_{c}:} H_{q}^{\prime \prime} \\
& =H_{q}^{\prime}\left(x, x^{\prime}-\frac{\partial H_{g}}{\partial x}+\frac{\partial H_{c}}{\partial x}, y, y^{\prime}-\frac{\partial H_{g}}{\partial y}+\frac{\partial H_{c}}{\partial x}\right)
\end{aligned}
$$

Equation (3.34) shows that I have separated the main effect of the CCS, the chromaticity correction with the Hamiltonian $2 H_{c}$, from the effect of the $-I$ brealidown represented by $H_{q}^{\prime \prime \prime}$. The study of this Hamiltonian shows that it indeed contains fiftl order aberrations. Using (3.30) and the definition of $H_{g}$ and $H_{c}$ one can rewrite (3.35) as

$$
H_{q}^{\prime \prime \prime}=\bar{\delta}\left(b x^{2}+a\left(x^{\prime}-\frac{k_{s}}{2}\left(x^{2}-y^{2}\right)+k_{s} \eta \bar{\delta} x\right)^{2}+d y^{2}+\left(\cdot\left(y^{\prime}+k_{s} \cdot y+k_{s} \eta \bar{\delta} y\right)^{2}\right)\right.
$$

The expansion of $H_{q}^{\prime \prime \prime}$ shows that the interaction of the quadrupole chromaticity with the geometric aberrations from the sextupoles generates three fifth order abcrations: $\bar{\delta} x^{4}, \bar{\delta} y^{4}$ 
and $\bar{\delta} x^{2} y^{2}$. The interaction of the quadrupole and sextupole chromaticities generates $\bar{\delta}^{3}, x^{2}$ and $\bar{\delta}^{3} y^{2}$. There are also two cross terms: $\bar{\delta}^{2} x^{3}$ and $\bar{\delta}^{2}: y^{2}$.

These fifth order aberrations are proportional to the a and c coefficients of (3.30), themselves originating mainly from the center quadrupole. I have also neglected all the terms in $x^{\prime}$ or $y^{\prime}$ since the angles are typically orders of magnitude smaller than the trajectory amplitudes at the FFTB sextupoles.

\subsubsection{Other uses of this formalism}

Having reduced the CCS under the form of a linear $-I$, a fifth order Hamiltonian and the main chromaticity tem, it is possible to insert it back into the FFTB beamline. Let me take the following model for the whole line, assuming only onc C'CS:

$$
e^{: H:}=e^{: H_{h}:} e^{: H_{5}:} e^{: 2 H_{c}:} e^{: H_{t}:}
$$

All Hamiltonians are expressed at the IP. The front-end of the line is modeled by the nonlinear Hamiltonian $H_{h}$ comprising the chromaticity of all elements from the beginning of the line up to the CCS. The part from the CCS to the IP is in $H_{t}$ and contains the very strong chromaticity of the final quadrupoles. Note that $H_{h}$ is very small compared to $H_{t}$.

The following transformation assumes that the chromaticity cancellation is perfect $\left(e^{: H_{h}}: e^{: 2 H_{c}} e^{: H_{l}:}=\right.$ Unity) and shows that the only remaining part in the total Hamiltonian of the line are some higher (fifth) order effects:

$$
\begin{aligned}
e^{: H:} & =e^{: H_{h}:} e^{: H_{5}:} e^{: 2 H_{c}:} e^{: H_{1}:} \\
& =e^{: H_{5}^{\prime}:} e^{: H_{h}:} e^{: 2 H_{c}:} e^{: H_{1}:} \\
& =e^{: H_{5}^{\prime}:}
\end{aligned}
$$

Note that $H_{5}^{\prime}$ has been transformed through $H_{h}$, howerer since the front end of the line contains very little chromaticity ${ }^{\star}$, the higher-order effects it could trigger combined with the fifth order effects are expected to be negligible. In other worels $H_{5}^{\prime} \sim H_{5}$. I will show in the next rhapter which are the conditions necessary for high order teme to become significant.

\footnotetext{
* I assume here only one CCS in the line
} 
There are, in conchusion, residual uncorrected fifth order abermations at the FFTB. Lhe cause of these terms is in the CCS and has been shown to be the interaction of the cluadrupoles chromaticity with both the geometric and chromatic abcrations of the sextupoles. Another significant term already mentioned is the fourth order long-sextupole aberration. This has been established without any numerical calculation, simply by analysis of the structure of the beam line and its components.

A systematic analysis such as that presented earlier in this chapter would give a global aberration picture of the line and would quantify the importance of these aberrations. All the power of the Lie algebra methods I present is here, allowing both the detailed and analytic expression of specific effects and their global sizing through the detailed calculations element by element.

\subsection{ERRORS AND OFFSETS}

The previous sections showed how to treat a perfect line. I want to show here how one can take into account clifferent displacements and errors in beam-line elements.

The connection between the similarity transformation and the displacement of an element is obvious:

$$
e^{:-d_{x} x^{\prime}:} e^{: H\left(x, x^{\prime}, y, y^{\prime}\right):} e^{: d_{x} \cdot x^{\prime}:}=\exp \left(e^{-: l_{x}, x^{\prime}:} H\right)
$$

and

$$
\begin{aligned}
e^{-: d_{x} \cdot x^{\prime}: H} & =H\left(e^{-: d_{x} x^{\prime}:} x, x^{\prime}\right) \\
& =H\left(x+d_{x}, x^{\prime}\right)
\end{aligned}
$$

The Hamiltonian is simply rewritten $H\left(x+d_{x}, x^{\prime}, y, y^{\prime}\right)$. Recall that $x^{\prime}$ stands for the function representing the trajectory and $d_{x}$ is a constant.

The introduction of offsets in the Hamiltonian gives rise to new terms. The corresponding aberrations are of order lower than that of the main term, they are called feed-down terms. For example the Hamiltonian of a horizontally displaced sextupole shows two new terms. A second-order term proportional to $d_{x}\left(x^{2}-y^{2}\right)$ representing a quadrupole effect and a first order term proportional to $d_{r}^{2}$. representing some horizontal stering. There is also a constant term that has no offect and is dropped here. In the presence of lattice dispersion the same displacement also generates adrlitional dispersion $\left(d_{x} \eta x\right.$ and $\left.d_{x} \eta y\right)$, feecl-down from the main 
chromaticity terms. This formalism actually shows clearly that dispersion is a by-product of the chromaticity of a displaced element.

Feed-down terms of order two and one are not a part of the design and cannot be included in the design linear optics of the line. They are therefore treated with the nonlinear Hamiltonians. The part of the optics that remains after the design linear optics has been removed now comprises non-linear as well as feed-clown terms and is called the "rest". The methods outlined in this chapter are not specific to non-linear optics and can accommodate these new terms.

It was possible to express the displacement formally as a similarity transformation because it affects directly one of the coordinate functions. Other errors like strength errors camnot be expressed in this way but can still be shown in the Haniltonian by directly clanging the constant: $K_{n} \rightarrow K_{n}+\Delta K_{n}$. There are now feed-clown terms generated by this error: an additional term with the same expression of the main term, and superimposed onto it. Strength crors for quadrupoles are treated as an additional quadrupole and are not treated in the design linear lattice.

Rotation errors and multipole content of an element can be represented as additional Hamiltonians inserted at the same location as the element. In the case of rotation error $(\phi)$, the Hamiltonian of the affected element is also subject to a strength error by reduction of the nominal strength $K \rightarrow K^{\prime} \cos \phi$.

It is of course now possible to study the effect on the optics of a single error in the line. The calculation of tolerances for the FFTB has been clone using these methods. They are the object of c apter 5 of this thesis.

Note finally that the displacement of a section or even the whole line can be studied by inserting the corresponding Hamiltonian in equation (3.39), loading to the calculation of tolerances for the section or, in the case of the whole line, to incoming bean tolerances.

\subsection{Conclusion}

This chapter has taken us from the general frmulation of the electronagnetic: Hamiltonian to the ability to analyze the optical effects in a beamline with great selectivity. The key points are the use of the similarity transformation and the C BH theorem. The similarity transformation allome ns to change the coordinates of a given Hamiltonian which loads to 
the possibility to separate the linear and nonlinear optics. It is also helpful in the handling of compensation mechanism such as that of the geometrics in the C'CS. The combination of remaining Hamiltonians is handled by the $\mathrm{CBH}$ theorem.

The same method can be applied from within a single elenent to obtain the Hamiltonian that takes into account the length effects, considering a subset of the line to locally eliminate some aberations from the calculation as in the treatment of the C'C'S, or at the level of the whole line to get the global picture of the aberration content of the line.

The similar treatment applied to the single element and the whole beamline shows how scalable these tools are. This is of great help in the understanding of the optics and will ultimately prove very helpful for the building of a computer code based on them.

The introduction of errors has been mentioned in the last section and I now turn in the next two chapters to an analysis of the aberration content and the tolerances for the FFTB using the now complete set of tools using Lie algebra. 


\section{Aberrations at the FFTB}

\subsection{INTRODUCTION}

Using the tools and methods developed in chapter 2 and 3, I analyze here the aberration content of the FFTB optics. The order of an aberration is to be understood in this chapter as the order of the corresponding monomial in the Hamiltonian. Therefore a third order aberration corresponds to a second order optical effect as defined in the Transport formalism, represented there by an entry in the $T$ matrix.

After a few remarks on the classification and the number of aberrations we have to consider, I show how one can apply a few simple arguments to find whether the effect of a given aberration can be important or on the contrary is negligible. This will allow us to effectively analyze only the important aberrations. Then I turn to the systematic analysis of all the important aberration terms at up to fifth order for the FFTB.

Remember that by nature Lie algebra acts on functions, not on coordinates like mapping or matrix tools. All references to "coordinates" in the frame of Lie algebra should therefore be understood as "coordinate functions". Let me also recall that I an using here three different sets of coordinate functions. The usual set $\left\{x, x^{\prime}, y, y^{\prime}\right\}$ refers to the local coordinate functions in a given beamline element. The usual coordinates at the IP are noted $\left\{x^{*}, x^{\prime *}, y^{*}, y^{\prime *}\right\}$. Finally the set of reduced coordinate functions, or "bar coordinates", is defined by the linear transformation:

$$
\bar{y}=\frac{y^{*}}{\sqrt{\beta_{y}^{*}}} \quad, \quad \bar{p}_{y}=y^{\prime *} \sqrt{\beta_{y}^{*}}
$$

and the corresponding formulas for the other plane. The Hamiltonian is unchanged by this scaling. Note that although I denote the conjugates of the position functions as $\bar{p}_{x}$ and $\bar{p}_{\boldsymbol{y}}$ they are not momenta. The position and angle functions defined by (4.1) are both in units of square root meters $\left([L]^{1 / 2}\right)$.

The fifth general coordinate is the momentum deviation of the particle $\bar{\delta}=\frac{p-p_{0}}{p}$. Since I do not consider acceleration or radiation effects in this study, $\bar{\delta}$ is a constant. Its conjugate, the time of flight, is not critical at the FFTB so the set of coordinate functions we use for the rest of this chapter is $\left\{\bar{x}, \bar{p}_{x}, \bar{y}, \bar{p}_{y}, \bar{\delta}\right\}$. 


\section{2. CA SSIFICATION}

Chapter 3 showed how to "remove" the first optical order from the problem of analyzing the optics with Lie algebra based techniques. This manipulation implies that all Hamiltonians describing individual elements are now expressed at the IP as polynomials in the set of $\left\{\bar{x}, \bar{p}_{x}, \bar{y}, \bar{p}_{y}, \bar{\delta}\right\}$.

The aberrations arising from the $\mathrm{C} B \mathrm{BH}$ combination of individual non-linear Hamiltonians, including the element length effects, exist in the line by design and I classify them as "inherent aberrations". They can be corrected, often by the insertion of specific correction lenses in the line. One purpose of final focus systems is to climinate all such design aberrations that can affect the spot sizes at the IP. The largest is the chromaticity introduced by the quadrupoles. This goal can be summarized by $\frac{\partial H}{\partial \bar{p}_{y}}=0$ and $\frac{\partial H}{\partial \bar{p}_{x}}=0$ if $H$ is the total non-linear Hamiltonian of the system.

In practice a beamline cannot be built exactly to design and wo have to consider what the consequences of errors are on the aberrations, as well as on the linear optics. Errors (displacement, strength error, rotation) can be expressed as additional Hamiltonians in the line, some of them at first and second order as seen in chapter 3. The consequence is that we now find, when combining the Hamiltonians, some terms at first and second order as well as new terms at higher order. I call these aberrations that arise becallse of errors in the line "induced aberrations". The study of the induced aberations is really a problem of tolerances and is the subject of the next chapter. I will however numtion some of them in the present chapter.

\subsection{NUMBER OF ABERRATIONS}

\subsubsection{Symplecticity}

Before looking at the precise number of aberrations we hare to investigate. it is interesting to make a comparison with the number of entries in the matrices representing the optic's in the Transport formalism. The matrices must obey the symplectic condition which specifies some relations between matrix elements. For a $2 n \times 2 n$ linear matrix $(R)$ the symplecticity condition is written

$$
R^{T} J R=J
$$


where the matrix $J$

$$
J=\left(\begin{array}{cc}
0 & I \\
-I & 0
\end{array}\right)
$$

is built using the two $n \times n$ matrices: $(I)$ identity or unit matrix and $(0)$ the null matrix.

It can be shown that for $2 \times 2$ matrices the symplectic condition is written: $\operatorname{Det} R=1$.

At higher orders the relation between matrix elements can also be seen starting from the Hamiltonian representation of the optics: As stated by Hamilton's equations, any non-zero derivative of $H$ with respect to one of the $\bar{x}, \bar{p}_{x}, \bar{y}, \bar{p}_{y}$ represents a change in the conjugate variable, and therefore an aberration. The same Hamiltonian can then represent several optical aberrations and they are related to each other. For example consider the third order geometric aberration $H=C \bar{x} \bar{p}_{x} \bar{p}_{y}$. There are three non-zero derivatives and three matrix elements generated by this Hamiltonian, together with two relations: $T_{114}=T_{224}=T_{312}$. The three matrix elements constrained by two relations are of course equivalent to one coefficient $(C)$ in the Hamiltonian.

The higher the order, the more relations one has to take into account, and it follows that a "catalog of aberrations" using Lie algebra methods requires less terms to keep track of, as compared to the Transport notation and methods, and there is no need to carefully establish the dependencies between the different optical aberrations in the matrices. ${ }^{[34]}$

\subsubsection{Numbers}

In order to evaluate the number of aberrations at a given order, one can naively start with the idea that all the possible monomials associated with geometric aberrations at order $n$ are in the polynomial ${ }^{\star} G_{n}=\left(\bar{x}+\bar{p}_{x}+\bar{y}+\bar{p}_{y}\right)^{n}$. The chromatic terms at order $n$ are added to this collection by taking the product of the energy deviation $\bar{\delta}$ with all the aberrations (chromatic and geometric) at order $n-1$. At order $n$ the list of all possible aberrations is then given, in the form of a polynomial by the recursive equation showing the separation between geometric and chromatic terms:

$$
\begin{aligned}
H_{n} & =\left(\bar{x}+\bar{p}_{x}+\bar{y}+\bar{p}_{y}\right)^{n}+\bar{\delta} H_{n-1} \\
\text { with } \quad H_{0} & =1
\end{aligned}
$$

* the coefficients of this polynomial do not hold any special meaning here: they are just coming from the expansion and can be ignored. 
The number of all the monomials in the expansion of 11 , variables at all orders 11p to $1 \|_{0}$ is

$N_{m}=\frac{\left(n_{v}+n_{v}\right) !}{n_{0} ! n_{v} !}$. At order up to five and for five variables we get a total of 252 mathematically possible terms. This seems like an impressive number if we want to calculate those terms by hand but there are some arguments to reduce this number to a more manageable level.

\subsubsection{Significant terms}

The first argument depends on the optical structure of final focus systems with the characteristic phase advance pattern; most elements are located $(\pi / 2+n \pi)$ away from the IP.

Expressed in local coordinates the aberrations we are looking at, coming from the potential part of the Hamiltonian, are expressed as polynomials in the $\{x, y, \bar{\delta}\}$ coordinates.

Upon linear transformation to the IP, the coordinates are transformed according to $x \rightarrow$ $a \bar{x}+b \bar{p}_{x}$ and $y \rightarrow a \bar{y}+b \bar{p}_{y}$. The phase pattern however ensures that $a / b=\operatorname{cotg} \Delta \phi \ll 1$ and it is therefore sufficient to consider the leading term in $\bar{p}_{x}$. In other words an aberration $x^{n}$ in local coordinates is transformed into the $\bar{p}_{x}{ }^{n}$ at the IP and we can neglect the other terms $\left(\bar{p}_{x}{ }^{n-1} \bar{x}_{,} \ldots\right)$. The only significant chromatic terms for example are $\bar{p}_{x}{ }^{2} \bar{\delta}$ and $\bar{p}_{y}{ }^{2} \bar{\delta}$ which happen to be corrected at the IP. The $\bar{x} \bar{p}_{x} \bar{\delta}$ and $\bar{y} \bar{p}_{x} \bar{\delta}$ are negligible.

This argument greatly reduces the potential number of aberration one has to investigate. In fact we can deñne the $G_{n}^{\prime}$ polynomials analogous to the $G_{n}$ defined above but expressed in the set of $\left\{\bar{p}_{x}, \bar{p}_{y}, \bar{\delta}\right\}$. This now gives a total number of 50 aberrations to be studied at order up to five.

There is finally the case of the two elements at the center of the two CCS which are "in phase" with the IP, more exactly $n \pi$ away from the IP. Aberrations from these quadrupoles are expressed at the IP as functions of the $\bar{x}$ and $\bar{y}$ coordinate functions $(H(\bar{x}, \bar{y}))$. Since they are of order zero in the $\bar{p}_{x}$ and $\bar{p}_{y}$ functions their Poisson bracket with these functions are identically zero $([H(\bar{x}, \bar{y}), \bar{x}]=[H(\bar{x}, \bar{y}), \bar{y}]=0)$ and there is no effect from these terms on the spot size at the IP. One might argue that the next lrading term, in this case $\bar{x}_{x} \bar{p}_{\bar{\delta}}$ and $\bar{y} \bar{p}_{y} \bar{\delta}$, do affect the spot size. However their coefficients are smaller than that of the main terms by a factor $a / b \gg 1$ and they are negligible.

The only remaining concern for these two elements is their possible interaction with other aberrations coming from elements at the other phase, sextupoles for example, as this 
can lead to higher order effects.

The second argument to reduce the number of term is the further removal of terms that do not have an effect on the beam size at the IP. We are already working with the $G_{n}^{\prime}$ polynomials so the only remaining such terms are the purely chromatic $\bar{\delta}^{n}$. The conjugate of the energy deviation is the time of flight coordinate but since the bunch length is not a critical issue at the FFTB we will simply drop these chromatic terms (6 terms at order up to 5).

Finally there are a few terms at lower order that we have to consider despite the first argument given above. The first order $H=\bar{x}$ and $H=\bar{y}$ for example represent the angular steering of the beam at the IP. They are not too critical for the FFTB where this effect is largely dominated by the dispersion correction scheme. Also two geometric terms $\overline{x p}_{x}$ and $\overline{y p}_{y}$ must be included. These terms are difficult to produce within the FFTB for the reasons exposed above but they could be present in the form of correlations in the incoming beam. They represent a $\beta$-function mismatch at the IP. The leading terms $\bar{p}_{x}{ }^{2}$ and $\bar{p}_{y}{ }^{2}$ represent a motion of the waist or $\alpha$-function mismatch at the IP. The Beta-Matching section at the entrance of the line will correct for these "aberrations".

Finally the count of the aberrations to be studied comes to 54 terms compared to the 252 we previously envisioned. And this number is only the number of possible terms, the number of actual aberrations involved is certainly lower.

\subsection{Summary}

Before turning to the systematic study of the FFTB aberrations the following table shows a summary of the situation. At first and second order we have only induced aberrations. At higher orders the distinction can be made between the geometrics and the chromatics. Recall that the chromatics can be built from the aberrations at one order lower times the momentum deviation $\bar{\delta}$. The geometric aberrations introduced at order $n$ correspond to the $2 n$-multipole principal term and all its variations ( $c f$. the polynomial $G_{n}$ above).

I have mentioned in broad terms the main aberration or the main source for each entry above. For example quadrupole under the term $G_{2}$ means not only the normal quadrupole aberrations (strength error, etc.) but also the skew quadrupole terms. The geometric term Gis comes from decapolar fields and should not be present at the FFTB as will be shown 


\begin{tabular}{|c|c|c|c|c|}
\hline :der l & 2 & 3 & 4 & 5 \\
\hline steering & quadrupole & sextupole & long sextupole & ? \\
\hline$G_{1}^{\prime}$ & $G_{2}^{\prime}$ & $G_{3}^{\prime}$ & $G_{4}^{\prime}$ & $G_{5}^{\prime \prime}$ \\
\hline & dispersion & chromaticity & second orcier chrom. & chromatic breakidoun \\
& $G_{1}^{\prime} \bar{\delta}$ & $G_{2}^{\prime} \bar{\delta}$ & $G_{3}^{\prime} \bar{\delta}$ & $G_{4}^{\prime} \bar{\delta}$ \\
& & $G_{1}^{\prime} \bar{\delta}^{2}$ & $G_{2}^{\prime} \bar{\delta}^{2}$ & $G_{3}^{\prime} \bar{\delta}^{2}$ \\
& & & $G_{1}^{\prime} \bar{\delta}^{3}$ & $G_{2}^{\prime} \bar{\delta}^{3}$ \\
& & & & $G_{1}^{\prime} \bar{\delta}^{4}$ \\
\hline
\end{tabular}

Table 4.1. The classification of aberrations at the FFTB up to fifth order in the Hamiltonians. The $G_{n}$ are polynomial at order $n$ representing the geometric aberrations only. The soure or the main effect for these aberrations is also mentioned at each order for both the geometrics and the chromatics. The geometric fifth-order terms figure in this table since they are mathematically possible at this point: The analysis will show that none of these terms can anpear.

later in this chapter. In the case of chromatic terms I quote only the dominant aberration at each order. The following discussion now details these aberration term by term.

\section{4.j. First and Second Order}

The linear, first and second order, design lattice of the line has been removed already so that the aberrations listed in this section are all induced, appearing only because of errors and misalignments.

At first order we have to consider not only the $\bar{p}_{x}$ and $\bar{p}_{y}$ terns which represent the steering of the beam at the IP, but also the $\bar{x}$ and $\bar{y}$ terms representing the angular steering. The angle of the beam at the IP is not very important at the FFTB since we have a one-beam experiment. In a real collider like the SLC where both beams share part of the machine this is an important parameter. At the NLC with separate exit channels for the spent beams, this parameter is not so important in the point of view of the optics. It is however a cause of luminosity degradation and potential source for high backgrounds as experienced at the SLC and therefore should not be neglected.

At the FFTB we have correctors close to the final quadrupoles in order to stecr the beam at the IP, or control the $\bar{p}_{x}$ and $\bar{p}_{y}$ terms. The angular steering terms will be clominated by the dispersion control mechanism. 
The number of geometric effects to consider at second order is also increased with respect to the rules laid out in the previous sections: On top of the three terms representing the waist motion and the principal coupling term (first three lines in table 4.2), I have added two terms representing the change of $\beta$-function at the IP. Finally two chromatic terms are present and can be built from the first order geometrics times the energy deviation. They represent the dispersion terms.

\begin{tabular}{|c|c|c|}
\hline monomial & status & origin \\
\hline $\bar{p}_{x}^{2}$ & quadrupole & quad. strength \\
\hline $\bar{p}_{y}^{2}$ & quadrupole & or sext. Horiz. offset \\
\hline $\bar{p}_{x} \bar{p}_{y}$ & coupling & quad. rotation or sext. Y offset \\
\hline $\bar{x} \bar{p}_{y}$ & negligible & \\
\hline $\bar{y} \bar{p}_{x}$ & negligible & \\
\hline $\bar{p}_{x} \bar{\delta}$ & dispersion & quad. offset \\
\hline $\bar{p}_{y} \bar{\delta}$ & dispersion & or dipole strength \\
\hline
\end{tabular}

Table 4.2. Catalog of the second order aberrations at the FFTB. The terms shown as negligible should not appear at the FFTB IP. They are beta-matching terms and should be treated in the appropriate section at the head of the beamline.

Let me now detail the origin and cure for these aberrations.

The leading geometric terms $\left(\bar{p}_{x}^{2}, \bar{p}_{y}{ }^{2}\right.$ and $\left.\bar{p}_{x} \bar{p}_{y}\right)$ are caused by strength errors in quadrupoles, rotation of quadrupoles or offsets in sextupoles. The first two are $\beta$-matching terms, the last one is a coupling term.

The $\bar{p}_{x}^{2}$ term for example represents a motion of the waist away from the nominal focal point; its effect is to introduce here correlations between $\bar{x}$ and $\bar{p}_{x}$ or to tilt the phase ellipse. In terms of twiss parameters it is a change of the $\alpha$-function. The $\bar{x} \bar{p}_{x}$ term on the other hand changes the widths of the distributions without affecting the orientation of the ellipse. This is interpreted as a change of the $\beta$-function in the corresponding plane.

The $\beta$-matching section (BM) and a skew quadrupole in the final transformer correct for these effects at the FFTB. The two terms $\overline{x p}_{x}$ and $\overline{l n}_{y}$ are small per the argument of 
th.. mereding sections, although they could arise from a mismatched beam at the entrance of the line. They can be corrected in the BM section.

The main coupling term $\bar{p}_{x} \bar{p}_{y}$ is corrected by placing a skew quadrupole in the dispersionfree region of the FT where both $\beta$-functions are high. The next two torms for coupling ( $\bar{x} \bar{p}_{y}$ and $\bar{p}_{x} \bar{y}$ ) correspond to a rotation of the beam in physical $x$ - y space but are small as we have shown.

They too can appear however in the inconing beam although, as mentioned in chapter 1 , it is expected that they will be small. Note that even in the BM section there is no location where we could place skew quadrupole to control this rotation of the beam in phase space efficiently.

The second order chromatic effects can be obtained by multiplying all the first order terms by $\bar{\delta}$. Only two significant terms appear here: the clispersion in both planes. This induced effect appears from offsets in quadrupoles, or other multipoles or if dipoles depart from their nominal setting. Generally dispersion arises whenever the beam is offset in a chromatic element. It is easy to see this by calculation of the similarity transformation $e^{-: d_{x} x^{\prime}}: e^{: H_{\xi}:} e^{: d_{x} x^{\prime}}$ where $H_{\xi}$ is the chromaticity Hamiltonian. Of course, as shown in chapter 2 , this is equivalent to intuitively replacing the function $x$ by the function $x+d_{x}$ in $H_{\xi}$.

Following this remark, dispersion correction can be applied by intentionally creating an orbit offset in a region with high chromaticity quadrupoles. At the FFTB we use this technique in the final triplet, creating a bump with maximmm offsct at the final quadrupoles and zero position offset, but some angular offset, at the IP. Large amounts of incoming or internally generated dispersion can be corrected using this technique; the equivalent of seven sigmas at the IP for the FFTB.

These second order terms are all induced effects and we can seo that after careful analysis we have to really worry about five of them: two for the motion of the waists, one coupling term and dispersion in both planes. 


\subsection{Method OF ANALYSiS}

Before embarking for the third and higher orders, let me outline here the general approach one might use for this kind of systematic studies. The mechanism can be divided in three steps.

- The background lattice

Consider first a lattice containing only simple bending magnets and quadrupoles. I first suppose that the chromaticity of any given quadrupole is not too strong. The study of the aberrations introduced here is simple as we can limit ourselves to the first order of the expansion of the $\mathrm{CBH}$ theorem when combining elements together. In other words there is no need to consider the interactions between elements, the sum of the different Hamiltonians is sufficient to represent the beamline. At this point most of the aberrations present are induced; They are mostly a first and second order problem as will be ontlined in chapter 5.

Table 4.3 lists the aberrations that can possibly be prescut in the line according to this model, separated in inherent and induced terms.

\begin{tabular}{|c|c|c|}
\hline monomial & status & origin \\
\hline $\bar{p}_{x}$ & induced & dipole setting, quad offset \\
$\bar{p}_{y}$ & induced & dipole rotation, quad offset \\
\hline $\bar{p}_{x}{ }^{2}$ & induced & quad setting error \\
$\bar{p}_{y}{ }^{2}$ & induced & (idem) \\
$\bar{p}_{x} \bar{p}_{y}$ & induced & quad rotation \\
\hline $\bar{p}_{x} \bar{\delta}$ & induced & quad offset, dipole setting \\
$\bar{p}_{y} \bar{\delta}$ & induced & quad offset, dipole rotation \\
\hline $\bar{p}_{x}{ }^{2} \bar{\delta}$ & inhereit & quadrupoles \\
$\bar{p}_{y}{ }^{2} \bar{\delta}$ & inherent & quadrupoles \\
\hline
\end{tabular}

Table 4.3. The aberrations arising from the presence of the background lattice. The only higher order term is the chromaticity of the quadrupoles.

- Chromaticity correction 
If we now assume that we have to worry about the very strong final guadrupoles. let us add to this picture two pairs of sextupoles in a dispersive region. They have to be placed and set so that their geometrie effects cancel and the total chromaticity of the line is zero in both planes. This can be achieved by looking at the simple sum of the Hamiltonians, in other words there are still no interactions between elements in this picture. This is the work realized by most matrix based optics code that perform a "matching" of the $T$ matrix to cancel a few terms.

Since the high chromaticity terms are laid out in view of this cancellation, they are all located at the same phase with respect to the IP. In other words, their Poisson brackets vanish and they cannot generate higher order terms.

- Interactions

Since the background lattice and the large terms themselves cannot generate higher order effects, it has to be their interactions that trigger those unwanted aberrations.

Let me first mention a general rule that is obvious when consiclering the Poisson bracket. The interaction of two Hamiltonians of order $n$ and $m$ respectively gives, through the Poisson bracket, a Hamiltonian of order $n+m-2$.

This simple rule shows for example that the cross terms between the sextupole geometrics and a quadrupole chromaticity are of order $(3+3-2=4)$ or octupole-like. Similarly, in order for a given Hamiltonian to trigger lower order terms, one has to take its Poisson bracket with a term of order one to effectively "degrade" the resulting order.

Since it is the interaction of those large terms with the background lattice that generates the unwanted higher order aberrations, the number and importance of such higher order terms is limited by the number and importance of the large terms present in the first place.

In particular once we have shown the presence of a new term coming from this Poisson bracket interaction, we could in principle take again the Poisson bracket of this new term with other elements and generate even higher order terms. But since this new term has for coefficient the product of the coefficients of its generators and one of them is not large, the resulting aberation is not expected to have a very significant effect. However this moderate effect could, if compled to a very strong third term generate another moderate effect but at eren higher order. 
But obviously there has to be an end to this argument since there are only a linited number of very strong elements in this lattice, mostly the four sextupoles and the final quadrupoles.

With these essential ideas laid out, let me turn back to the systematic study of the FFTB lattice.

\subsection{THIRD ORDER}

The only significant sources of third order geometric aberrations at the FFTB are sextupoles. For this reason they are placed in pairs separated by a $-I$ module as shown in chapter 1 and 3 . There are no third order geometric aberrations remaining at the IP. They cannot inherently appear from interactions between elements since we have removed the linear optics, second order, already. They can appear if induced by a detuned quadrupole inside the $-I$ modules. The tolerances are estimated in the next chapter.

Third order chromatic aberrations are built from the aberrations at second order times $\bar{\delta}$. The main terms are of course the chromaticities in both planes, $\bar{p}_{x}{ }^{2} \bar{\delta}$ and $\bar{p}_{y}^{2} \bar{\delta}$, arising in quadrupoles as well as sextupoles located in a dispersive region. Chromaticity correction at the FFTB ensures that these terms vanish.

A careful study of the aberration picture at the FFTB shows that the above statement about the chromaticity terms vanishing is not true for the horizontal plane. There is some residual horizontal chromaticity at the IP. This is needed to achieve the maximum bandwidth and is found using a computer-based optimization. Since the chromaticity alone would reduce the bandwidth, this shows that there must be one or more other terms which are not exactly cancelled and whose effect counteracts to some extent that of the chromaticity term.

A likely candidate is the so-called second-order dispersion $\bar{p}_{x} \bar{\delta}^{2}$ which appears from the chromaticity of a quadrupole placed in a dispersive region. The main chromaticity term is, in local coordinates, $x^{2} \bar{\delta}$ where $x$ is really the transverse displacement of the particle which is composed of a geometric as well as a dispersive part. Changing $x$ into $x+\eta \bar{\delta}$ in this chromatic term reveals the second-order dispersion. This term is small but not negligible. Since there is no vertical dispersion in the line, there cannot be a vertical second-order dispersion term. The following shows the value of the dispersion function across the energy bandwidth at the FFTB, clearly revealing the second as well as third order dispersion. 


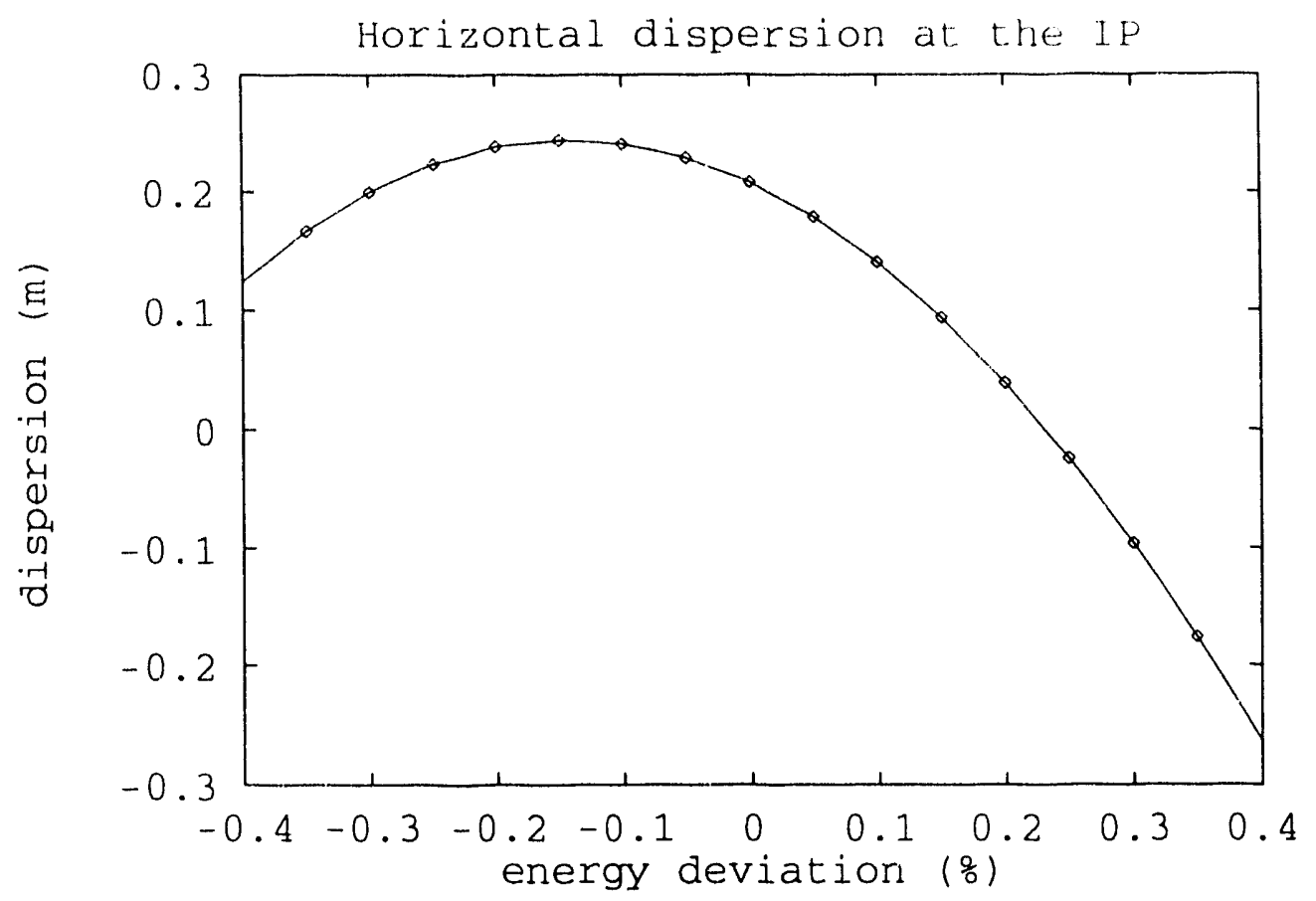

Figure 4.1. Horizontal dispersion at the FFTB IP across the energy bandwidth of the line. The slope at the origin indicates a second-order dispersion term while the curvature shows a thirdorder dispersion effect.

Note that there is some linear dispersion present at the IP according to this graph. The beamline used to obtain this data was an early version (FFTB68) and was not exactly matched. The contribution of this dispersion pattern to the IP spot size is only $0.01 \mu^{2}$ or an increase of the horizontal spot size of about $0.5 \%$. The vertical spot size is not affected.

This second order dispersion is indeed the only inherent third order aberration in the FFTB design. All other aberrations are cancelled or simply do not appear. Note finally that this aberration is inherent to the design of the FFTB and camnot be clininated. In other designs by $\mathrm{K}$. Brown and R. Helm, this effect is cancelled by adding to the symmetry of the system. The formation of $2 \pi$ modules for the CCS in the Brown-Helm design allows for the suppression of the BX section, so that the CCS are the only sections with non-zero horizontal dispersion. Inside the CCS all the quadrupoles are paired with a $-I$ transform in between and the same dispersion function at both quadrupoles. Each pair of quadrupoles locally cancel the second order dispersion and the net effect disappears. This is essentially the case at the FFTB inside the CCS. Outside the CCS however every quadrupole in a dispersive region contributes to the second-order dispersion. 
The suppression of this term using the symmetry of Brown-Holm ailows for a simpler optics design where one does not have to rely on a cancellation of two different effects to achieve the desired momentum bandwidth. The number of elements is higher and the overall length is increased however, one reason why this design was not chosen for the FFTB in the first place. It seems that final focus systems for the next generation of linear colliders would benefit from a more symmetric design, allowing the total cancellation of all inherent third order aberrations.

\subsection{FOURTH ORDER}

There are no octupole or higher order multipole at the FFTB so geometric fourth order effects have to arise from interactions. The only possibility is the interaction of two sextupolar geometric effects. More exactly it is the effect of the length of the sextupoles that appears here. This effect has already been discussed in chapter 1 and is described in detail in appendix B. Since it is exactly of octupolar form it could be easily corrected by the insertion of an octupole in the final tranformer for example. The effect is small enough however that we have decided not to implement the correction.

The skew octupole terms on the other hand camnot appear at the FFTB, except as induced by multipole errors in quadrupoles or other magnets.

In principle a fourth order geometric effect could also arise from the interaction of two different sextupoles but since they are placed in pairs at the FFTB, such an interaction is impossible. Another possibility would be the feed-down of a decapolar field in conjunction with a first order steering effect, effectively steering the beam off-axis in the decapole. But there are no such magnets at the FFTB.

Fourth order chromatic aberrations are a little more complicated. There are several potential sources:

- Quadrupole-Quadrupole interaction

The chromaticities of two quadrupoles located at different phases can trigger a term of the form $\left[\bar{p}_{x}{ }^{2} \bar{\delta}, \bar{x}^{2} \bar{\delta}\right]=-4 \bar{p}_{x} \bar{x}^{2}$ but due to the presence of the $\bar{x}$ this effect is expected to be small. This new term is a variation of the second-order chromaticity term $\bar{p}_{x}{ }^{2} \bar{\delta}^{2}$. The same term in the other plane appears also.

- Quadrupole-Sextupole interaction 
The simm effect can appear from the interaction of a quadrupole with the chromaticity of the sextupoles, with the same comment on the strength.

Actually this result is obvious when one considers the treatment of the CCS using similarity transformation presented in chapter 3. Equation (3.36) shows five fourth order terms

arising in the CCS: $\bar{x} \bar{p}_{x} \bar{\delta}^{2}$ and $\overline{y p}_{y} \bar{\delta}^{2}$ have already been identified. Also appearing are the interactions with the sextupole geometric aberrations: $\bar{x} \bar{p}_{x}{ }^{2} \bar{\delta}, \bar{x}_{\bar{p}}{ }^{2} \bar{\delta}$ and $\bar{y} \bar{p}_{x} \bar{p}_{y} \bar{\delta}_{\text {. }}$

But following arguments presented earlier in this chapter, the effects of these fourth order aberrations will be small owing to the fact that they all contain a $\bar{x}$ or $\bar{y}$ component.

\subsection{FIFTH ORDER}

As I recalled in the preceding paragraph, the whole CCS has already been treated in chapter 3 and equation (3.36) showed already the apparition of the fifth order aberrations. They are all important in the sense that none of them contains a $\bar{x}$ or $\bar{y}$ factor.

The other way to see those aberrations appear is to consider the fourth order aberrations of the preceding section and write their Poisson brackets with the strong chromatic aberrations of the final quadrupoles.

The two treatments are of course equivalent and correspond to two different ways to take the chromaticity into account. From the source point of view with the final quadrupoles or from the correction point of view with the analysis of the CCS.

Of course we have no decapoles at the FFTB and therefore no geometric fifth order aberrations are present.

\subsection{HIGHER ORDER}

In order to get even higher order effects, we need to identify another strong source of aberrations. However the fifth order we have just described alroady takes into account all the large aberrations at the FFTB: the sextupoles and the final quadrupoles with the central quadrupoles of the CCS. There is just no possible combination that would give a sizable aberration of order higher than five in this line. Of course it is in theory possible to get very high order terms given a sufficient number of aberrations at third and higher order and these terms certainly exist in the case of the FFTB if onc takes into account the exact 
phase advance of all the elements. However the precise phase adrance adjustment required for the chromaticity correction and to avoid the geometric aberrations form the sextupoles is enough to guarantee that these terms at order higher than five are minuscule, by ensuring that the large terms are in the right location, at the "right phase".

\subsection{CONCLUSiON}

This chapter has exposed the approach made at the FFTB in order to evaluate the aberration content of the line. Some important results have been given, both for the FFTB and in view of a NLC:

At third order, the imperfect symmetry of the FFTB leaves the second order horizontal dispersion term uncorrected. I have mentioned that a solution to this problem is to adopt a higher symmetry lattice using $2 \pi$ modules for the C'CS and suppress the non-symmetric: parts of the line: the dispersive parts of the Beta-Matching and the Final Transformer as well as the Beta Exchange section.

This would allow the correction of all inherent third order terms. At fourth order the long-sextupole aberration seems impossible to avoid. The correction of this aberration could be done with an additional octupole corrector. Other fourth order aberrations could be suppressed if we could change the linear lattice in order to avoid having a quadrupole placed at the wrong phase with respect to other elements. This would also suppress the fifth order terms. It is interesting to see that to some extent the high-order aberration content of the line is so much dominated by the linear lattice. The same is true for tolerances which we will consider in the next chapter. 


\section{Stability Tolerances.}

\subsection{Tolerance Budget.}

We have chosen a criterion for the allowed increase in spot size from the FFTB aberrations. Since we expect about five aberrations in each plane and the experience with measuring small spots at the interaction point of the SLC shows that it is possible to measure a relative change of $10 \%$ in the size of the beam, the maximum allowed increase per aberration is set at $2 \%$. We believe, following recent SLC experience, that with the ability to detect a $10 \%$ clange in the spot size it is possible to tune out an aberration clown to the $2 \%$ level.

This $2 \%$ IP spot size increase criterion, with $\sigma_{0}$ the nominal spot size and $\Delta \sigma$ the contribution of some aberration, can be expressed as

$$
\left(\sigma_{0}^{2}+(\Delta \sigma)^{2}\right)^{1 / 2} \leq 1.02 \sigma_{0}
$$

which translates into

$$
\Delta \sigma \leq \frac{1}{5} \sigma_{0}
$$

It is convenient to apply a linear transformation to the preceding formula back to the location where the aberration appears, i.e. where the kick occurs, which leads to an equivalent condition to the $2 \%$ criterion:

$$
\left(\Delta x^{\prime}\right)_{r m s} \leq \frac{1}{5} \sigma_{x}
$$

Now the coordinates are local and we compare an RMS value of the kick to the local divergence of the beam.

In terms of Hamiltonians, a kick is the action of a Hamiltonian on the coordinates of the

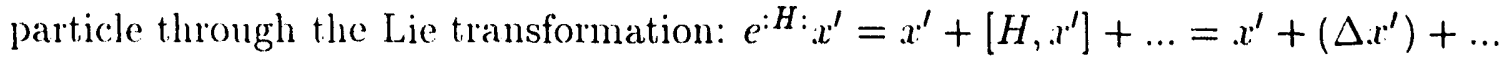

Therefore if the Hamiltonian form of an aberration is known, one can derive the condition ensuring that this aberation does not enlarge the spot size by more than $2 \%$ of the linear value. 
At the FFTB the total beam size growth above design is then expected to be $8 \%$ in the horizontal plane ( 4 contributing terms) and $14 \%$ in the vertical plane ( $T$ contributing terms). In the following discussion we will quote tolerances according to this $2 \%$ criterion for individual elements. However as different elements can contribute to the same aberration and if one assumes that their departures from design are not correlated, one must combine their tolerances in quadrature to find the tolerance for this group, $t_{g}$, which give a $2 \%$ increase in spot size:

$$
\frac{1}{t_{g}^{2}}=\sum_{i \in g} \frac{1}{t_{i}^{2}}
$$

where $t_{i}$ is the $2 \%$ tolerance for each individual element. We also refer to $t_{g}$ as the RMS tolerance for the group.

We usually separate the elements into two or more sets from most sensitive to least sensitive, and can allocate a fraction of the $2 \%$ budget to each group, the largest fraction to the most sensitive group. Within each group, $g$, we can then calculate an RMS tolerance for the group, $t_{g}$ according to

$$
\frac{1}{t_{g}^{2}}=\left(\sum_{i \in g} \frac{1}{t_{i}^{2}}\right) \frac{1}{f_{g}}
$$

where $f_{g}$ is the fraction of the $2 \%$ allocated to this group.

\subsection{Steering.}

Our tolerances on steering permit beam centroid motion at the interaction point to be one standard deviation of the horizontal and vertical distributions i.e. we allow the spot to move by $\Delta x^{*} \approx \sigma_{x}^{*}$ and $\Delta y^{*} \approx \sigma_{y}^{*}$.

This criterion is clifferent from the one quoted above and would clearly not be sufficient for a collider where the beams "ould miss each other by too much. Howerer at the FFTB we have only one beam and the Orsay Beam Size Monitor ${ }^{[37]}$ is insensitive to the position of the beam at the IP. Other methods such as the Laser-Compton monitor proposed by T. Shintake (KEK) or the liçuid wires developed by F. Villa (SLAC) are sensitive to the spot position at the IP and a movement of no more than a fifth of the spot size should be allowed. The scaling of tolerances for steering aberrations is linear. 
The expansion of the cuadrupole Hamiltomian, in the presene of a displacenent $d_{x}$. $H_{q}=\frac{1}{2} k_{q}\left(\left(x+d_{x}\right)^{2}-y^{2}\right)$, gives the Hamiltonian of the "stereing aberation" : $H_{s t}=k_{q q} d_{x} \cdot l^{\prime}$ and the corresponding kick $\left[H_{s t}, x^{\prime}\right]=k_{q} d_{x}$ giving the condition $k_{y^{\prime}} d_{x} \leq \sigma_{y^{\prime}}$ or

$$
d_{x} \leq \frac{1}{k_{q}} \sqrt{\frac{\epsilon_{x}}{\beta_{x_{q}}}}
$$

and similarly

$$
d_{y} \leq \frac{1}{h_{q}} \sqrt{\frac{\epsilon_{y}}{\beta_{y_{q}}}}
$$

The individual tolerances are presented on figure 5.1 for the horizontal plane and figure 5.2 for the vertical plane. Ignoring for now the final lenses $(Q \mathrm{C} 2, \mathrm{QX} 1, \mathrm{QC} 1$ and $\mathrm{FQ}$ on the graphs) the tolerances range from 1 to 10 microns in the horizontal plane and from 0.5 to 5 microns in the vertical. Note that the two quadrupoles located at the midpoints of the two C'CS have loose tolerances due to their phase relations ( $1 \pi$ ) with the IP. Taking the RMS value as defined above for these tolerances the R.IS tolerances for all guadrupoles except the finai triplet are 0.75 microns and 0.2 microns for the horizontal and vertical planes lespectively:

The case of the final cuadrupole triplet can be treated separately as these lenses are strongly tied together ${ }^{[3]}$ and the dominant motion will be one of all quadrupoles moving in the same direction and by the same amonnt.

A rery simple model of this final triplet is that of a non-realistic single optical lous focusing a parallel bean in both planes. The soberances on the notion of the lens is then equal to the criterion we choose. in other words a given moremont of the final lens moves the beam by the same amount at the focus $\left(\left(d_{x}\right)_{f_{q}} \approx \sigma_{x}^{*}\right.$ and $\left.\left(d_{y,}\right)_{f_{q}} \approx \sigma_{y}^{*}\right)$. This simple model gives estimates for the tolerances on the final quadrupoles motions of the order of 1 micron and 60 nanometers for the FFTL.

A more detailed calculation uses the fact that stering is a linear effect and one can simply sum, or actually integrate. the effects of all there icouses when displacer by the same annonnt :

$$
\Delta . r^{*}=\int d s k_{i q}(s) d d_{x} \sqrt{3,(s) \cdot 3_{x}^{*}} \sin \Delta \sigma^{* \rightarrow *}=\int d s k_{i g}(s) d d_{x} R_{12}^{*} \rightarrow^{*}
$$

and a sinilan expression for the oflece plane. The toleranes for the final cuadrupoles taken 


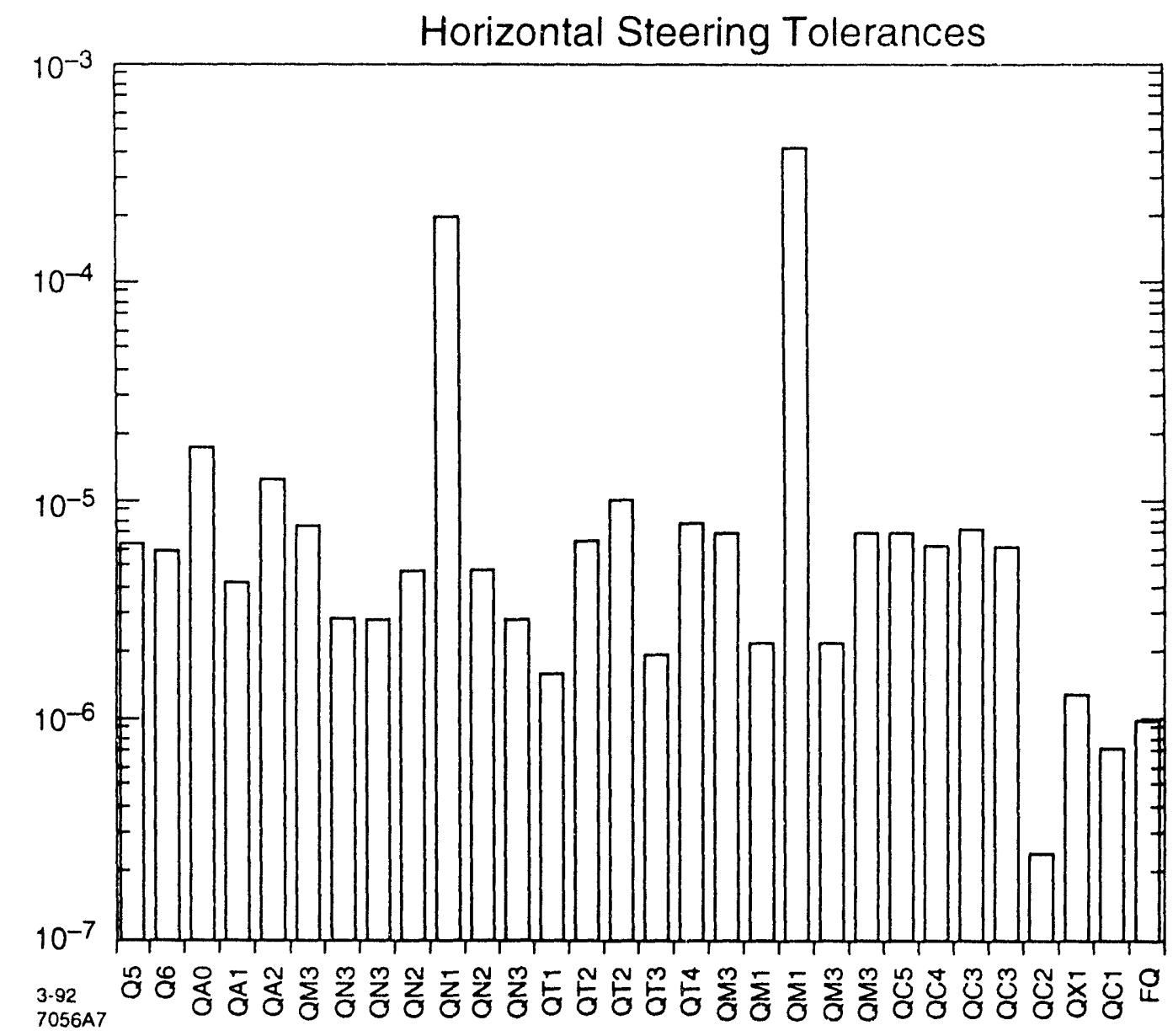

Figure 5.1. Horizontal steering tolerances at the FFTB. in meters. These values for individual elements stability tolerances correspond to a steering of one sigma of the bean distribution at the IP. The value quoted for the final quadrupoles taken as a single lens ( $F(2)$ correspond to the simple minded optical nodel montioned in the text. approximating the final lenses to a palallel-to-point focusing system.

as a single mint are extremely alose to those obtained thromgh the simple model above or $d_{r} \leq 1 \mu$ and $d_{y} \leq 6() m m$.

The steering tolerances are indeed very tight but it sluonde be (muphesized that at least one of the foreseen mothod of detemining the spot size at the FFTB, the ()rsay BSM. is insensitive to the bean position. The Compton-Laser spot size monitor will be attached directly to the final quarlinpole support table so motion of the final lenses shomld not affect it either. Exclucling the final cloublet we expect that the spot position at the IP will be dominater be the bean jitter from the linar. We have the ability at the FFTB to control

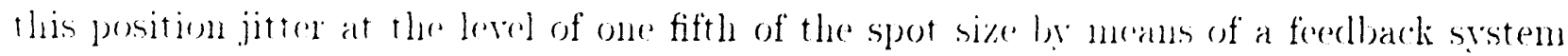
after the and of the linar. Slower drifts of the spot position at the IP an be controlled by 


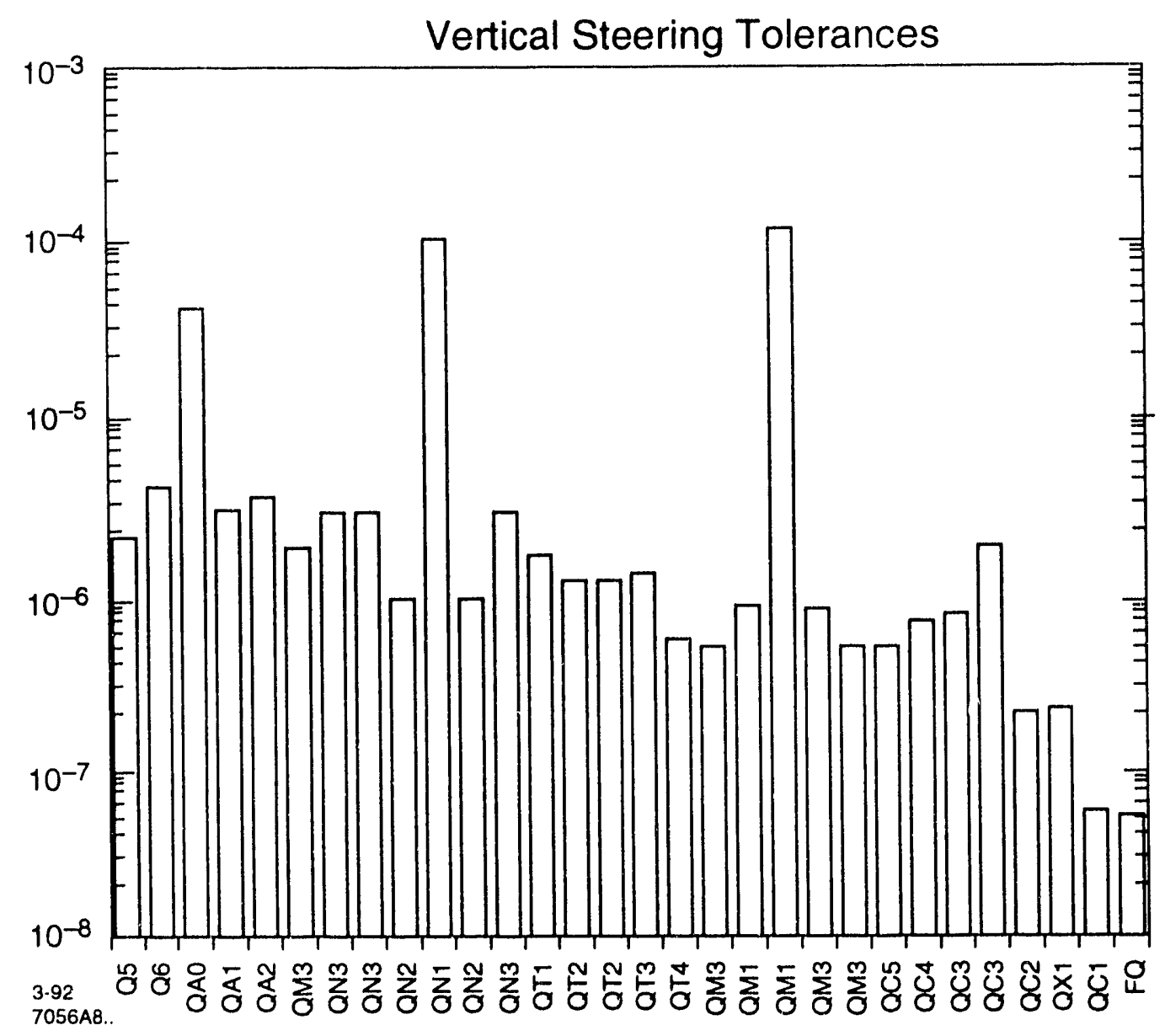

Figure 5.2. Vertical steering tolerances at the FFTB, in meters. These values for individual stability tolerances correspond to a steering of one sigma of the beam distribution at the IP. The value quoted for the final quadrupoles taken as a single lens (F(2) correspond to the simple minded optical model nentioned in the text.

feedback using a pair of air-core correctors close to the final (quadrupoles.

\subsection{Dispersion.}

Dispersion arises from an offset of the beam in cuadrupoles and is a consequence of the chromaticity of the quadrupole. The Hamiltonian of a cuadrupole including chromatic: effects is $H_{q}=\frac{1}{2} \frac{k_{q}}{1+\delta}\left(x^{2}+y^{2}\right)=\frac{1}{2} k_{q}\left(\ldots-x^{2} \bar{\delta}+y^{2} \bar{\delta}+\ldots\right)$ with the second form showing onty the chromaticity terms. In the presence of a displacement $d_{x}$, the Hamiltonian becomes $H_{q}=\frac{1}{2} k_{q}\left(\ldots-r^{2} \bar{\gamma}-2 d l_{x} x \bar{\gamma}+\ldots\right)$, whicin shows the dispersive term $H_{d}=k_{q} d_{x} x \bar{\delta}$.

The $2 \%$ ariterion is then written $k_{q^{\prime}} l_{x}(x \bar{\delta})_{r m,} \leq \frac{1}{5} \sigma_{x^{\prime}}$ or. since there are no more corre- 


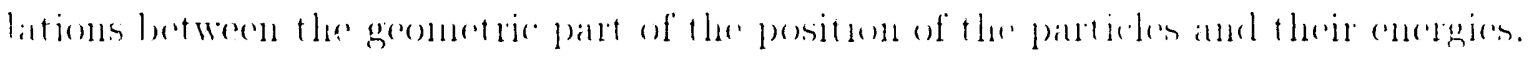

$$
d_{x} \leq \frac{1}{j k_{q} \bar{\delta}_{r m s}} \sqrt{\frac{\epsilon_{r}}{\hat{s}_{r_{q}}}}
$$

\section{Horizontal Alignment Tolerances}

(dispersion and normal quad. effects)

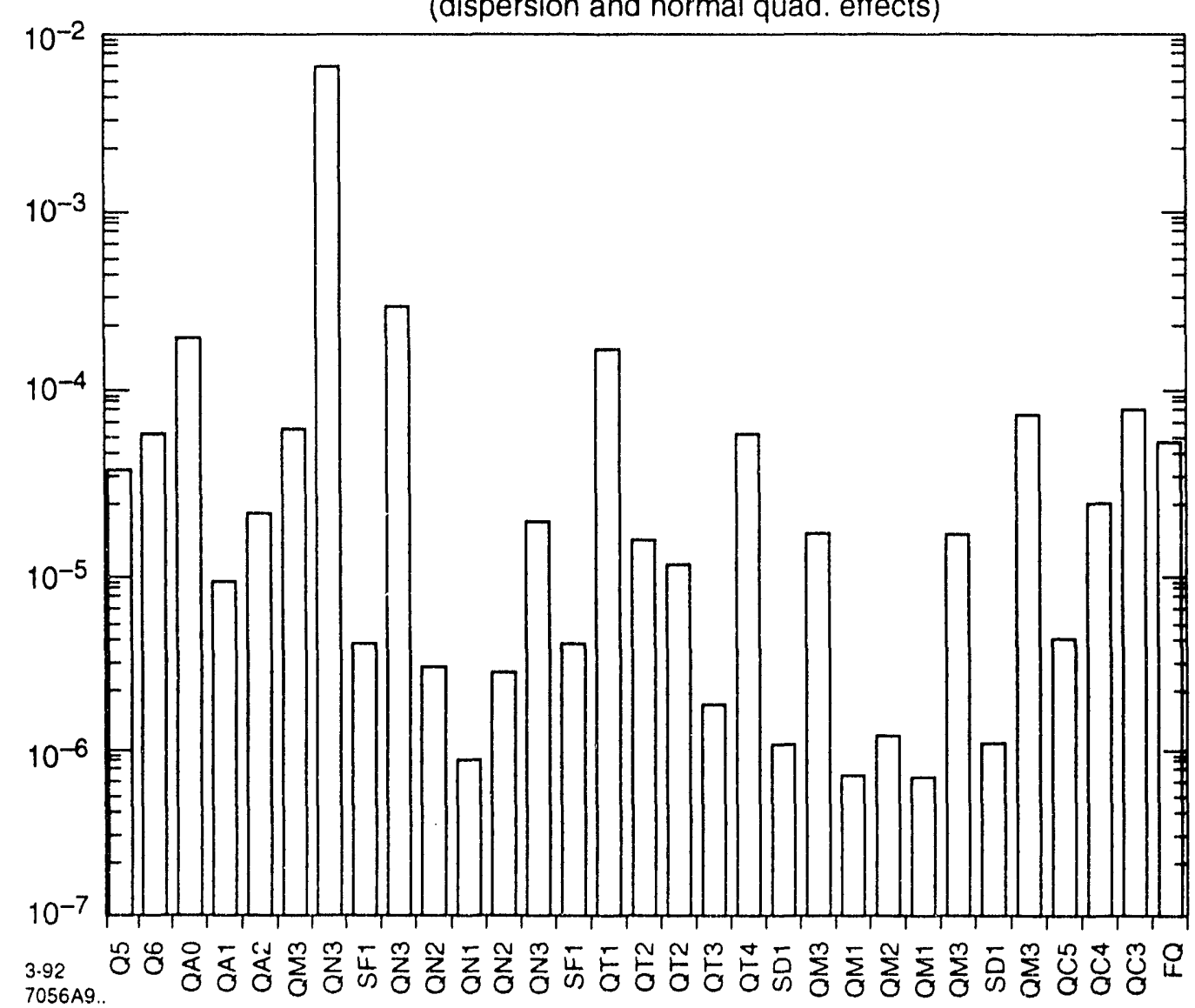

Figure 5.3. Horizontal aligmment tolerances at the FFTB. in meters. These values include the effect of dispersion both created directly and as a consecfuence of the orbit oscillation launched

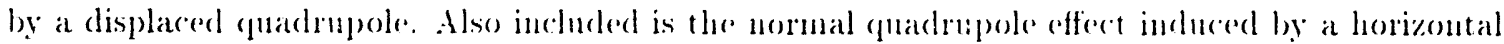
offset in sextupoles. This second offect dominates the tolerancess for sextupoles and the clements within both C'C'S. 


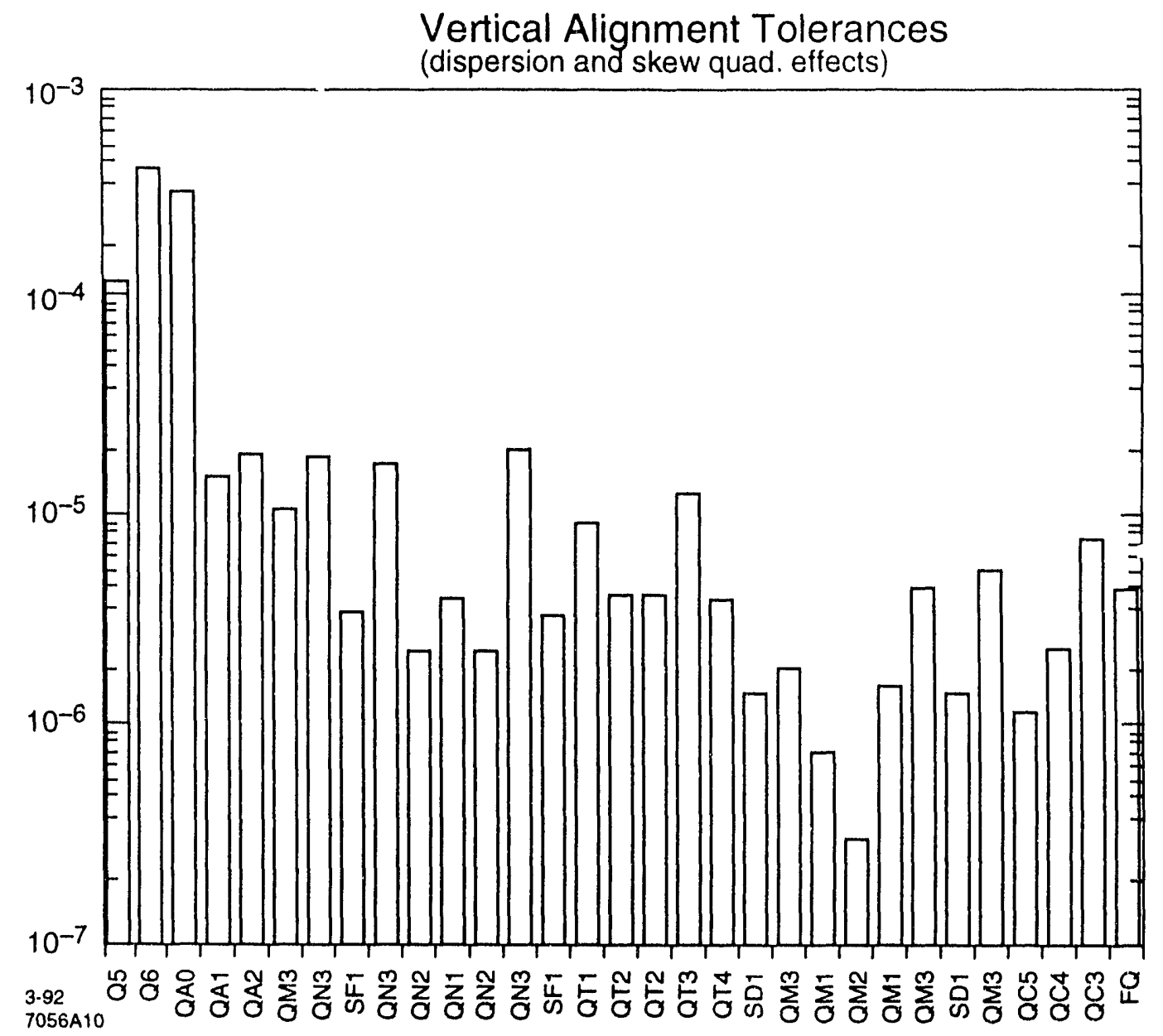

Figure 5.4. Vertical alignment tolerances at the FFTB, in meters. These values include the effect of dispersion both created directly and as a consequence of the orbit oscillation launched by a displaced quadrupole. Also included is the skew quadrupole effect induced by a vertical offset in sextupoles. This second effect dominates the tolerances for sextupoles and the elements within both CC.S.

\subsubsection{Final quadrupoles}

Under the same model of a single lens focusing parallel to point, we lave $k_{q} \sim \frac{1}{f}$ the inverse focal length of the system and the above expression can be approximated by

$$
d_{x} \leq \frac{1}{5 \bar{\delta}_{r m s}} \sigma_{r}^{*}
$$

Note that cren the formula (5.9) above is only an approximation of the correct expression becanse the Hamiltonian we considered in the first place is a kick approximation and does not take into acromut the thick lens effects which are particularly important for the final 
quadrupoles. 'Taking this into alceonut one gets

$$
d_{. r} \leq \frac{1}{5 \delta_{r m s}} \frac{\sigma_{x}}{\xi_{. r}}
$$

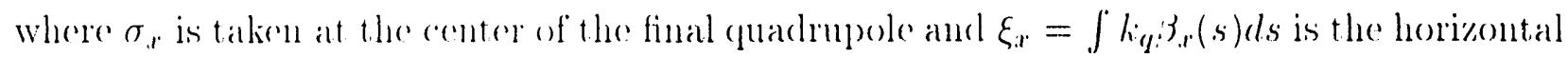
chromaticity of the final guadrupole.

\subsubsection{Other (quadrupoles}

The offset at the final quadrupole can be created by a direct movement of the final quadr":ipole or by a displacement of another quadrupole upstream steering the beam off-axis in the final lens. To study this second effect we introduce the notion of lattice multipliers defined as the amplification factor between the offset of a given cquadrupole and the centroid offset in the final (unadrupole. Note that the two displacements have opposite signs in the hamiltonian, hence the minus sign in the following formula.

$$
\left(l_{x}\right)_{f_{q}}=-\left(k_{q} R_{q \rightarrow f_{q}}^{12}\right)\left(l_{x}\right)_{q}
$$

Lattice multipliers depend only on the lattice structure, not on the focal point parameters or the bean properties. The greater this multiplier the tighter the dispersion tolerances on the dement.

For most (puadrupoles at the FFTB this second part is dominant over the direct dispersion generated by the displaced (quadrupole itself. In fact the lattice multipliens are so large in the FT that we use this property to globally ancel dispersion at the IP: Intentionally creating a controllod orbit offset in the final lenses gives us the ability to generate up to seven sigmas of clispersive (ontribution to the beam at the IP. Of course this bump is not closed across the IP and the beam is at an angle there. The closure is easily clone in the dump line. This is not a problem for the FFTB where wo have only one beam. It would canse a loss of luminosity for a real collider. 'The SLC' is very sensitive to this effect for masking reasons since the ontgoing beam must travel on the same path as the opposite incoming bean. Future linear colliders will have sepanate exit patles for the ontgoing heam and therefore the situtaion is very similan to that of the FFTB. 


\subsubsection{Sextupoles}

Sextupoles are also chromatic elements in the presence of a main dispersion term and their displacement, either directly or by orbit offset, is therefore source of dispersion. The Hamiltonian of a sextupole in a dispersive region is $H_{s}=\frac{k_{s}}{3 !}\left(\ldots+3 \eta_{x} \bar{\delta} x^{2}-3 \eta_{x} \bar{\delta} y^{2}+\ldots\right)$ and translates into $H_{s}=\frac{k_{x}}{3 !}\left(\ldots+6 \eta_{x} \bar{\delta} d_{x} x-6 \eta_{x} \bar{\delta} d_{y} y+\ldots\right)$ in the presence of displacements $d_{x}$ and $d_{y}$.

This effect through orbit offsets is to be added to the previous ones when determining the alignment tolerance of a given quadrupole.

The figures 5.3 and 5.4 present the results of these tolerances calculations. For quadrupoles outside the CCS, these numbers are those for dispersion only. One notices that the front quadrupoles have very loose tolerances. This is obvious since the chromaticity from these front quadrupoles to the IP is corrected, therefore an orbit oscillation will produce dispersion in each chromatic element but these different contributions also cancel each other at the IP. On the other hand, as already mentioned, the tolerances for a few elements in the FT are very tight since the chromaticity from there to the IP is only that of the final quadrupoles and is not cancelled.

For quadrupoles inside the CCS, these graphs present also another effect which is analyzed later. Horizontal and vertical beam offsets in sextupoles also produce quadrupole and skew quadrupole components. These effects in fact dominate the tolerances for those elements situated within a sextupole pair and the associated tolerances are derived in section 5.5.2 and 5.7.2 .

\subsection{Normal Quadrupole.}

A change in quadrupole strength will result, for most quadrupoles at the FFTB, in a movement of the waist away from the focal point and cause an increase of the spot size at the focal point. The Hamiltonian representing this aberration is $H=\frac{1}{2} \Delta k\left(x^{2}-y^{2}\right)$ giving a kick $\Delta x^{\prime}=\Delta k x$. The $2 \%$ increase criterion for the horizontal plane is

$$
\begin{aligned}
\Delta k(x)_{r m s} & \leq \frac{1}{5} \sigma_{x^{\prime}} \\
\Delta k & \leq \frac{1}{5} \frac{\sigma_{x^{\prime}}}{\sigma_{x}} \\
\Delta k & \leq \frac{1}{5 \beta_{x}}
\end{aligned}
$$


and finally the strength tolexance is, considering both planes:

$$
\frac{\Delta k}{k} \leq \frac{1}{5 k \operatorname{Max}\left(\beta_{r}, \beta_{y}\right)}
$$

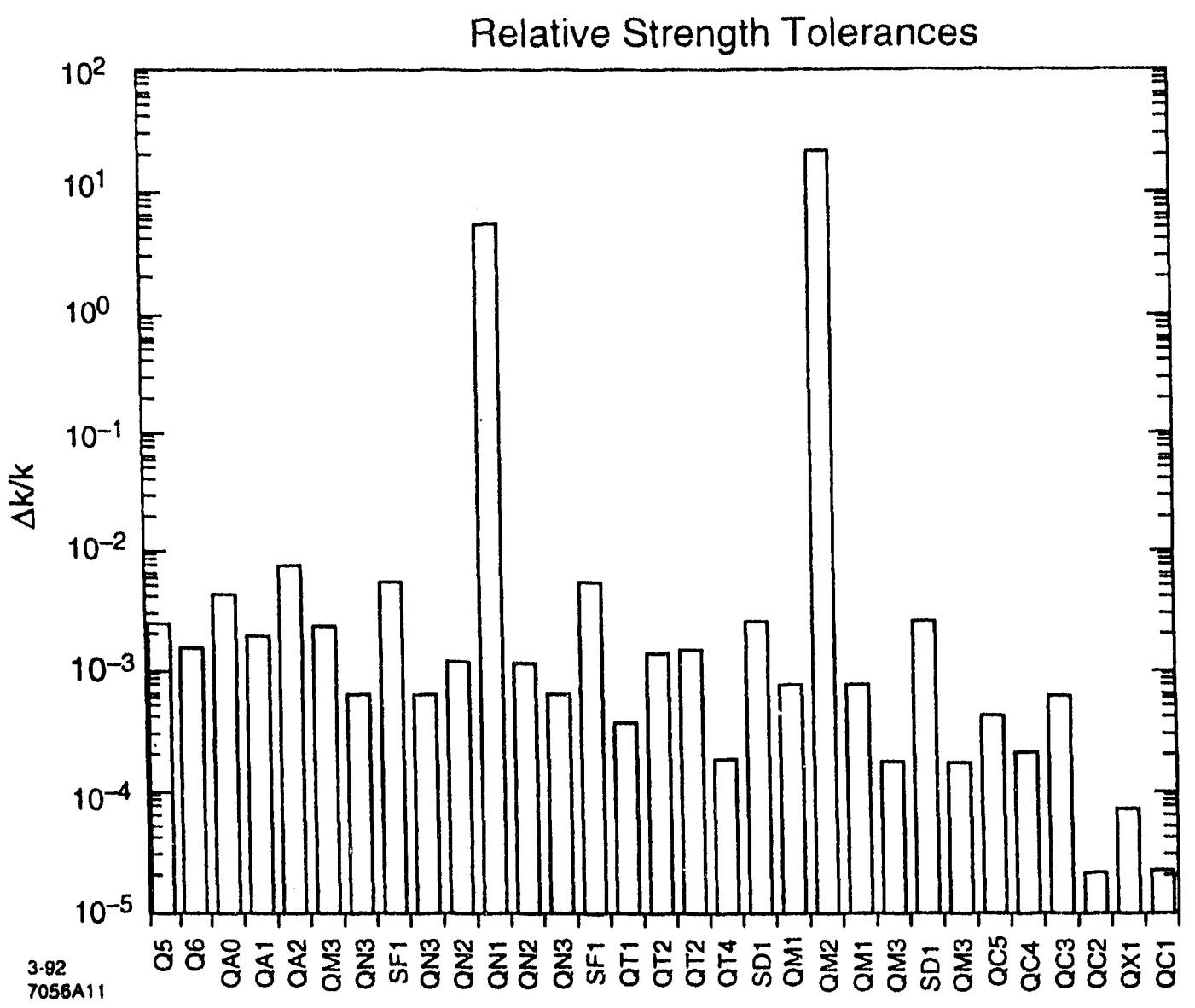

Figure 5.5. Strength stability tolerances at the FFTB. Note that the expression of these tolerances for sextupoles is not derived in the text and corresponds to individual sextupole tolerances. Placing each pair of sextupole on a single power supply would increase the tolerances accordingly.

Individual tolerances are shown on figure 5.5. The final guadrupoles have the tightest tolerances as expected; the strength of $\mathrm{QC} 2$ and $\mathrm{QC} 1$ must be controlled at the level of $\Delta h / h=2 \cdot 10^{-5}$, recpuiring special power supplies. Other quadrupoles in the line typically have tolerances of the order of a few $10^{-4}$ up to a few $10^{-3}$. The tightest tolerances for quadrupoles other than the final lenses are achieved for those elements around the sextupoles in the CCY with $\Delta k_{i} / k \sim 1.710^{-4}$. The RMS tolerance for all quadrupoles except the final triplet is $\Delta k: k=7.310^{-5}$ which is a tight value for usual magnet power supplies. 
Howeree these tolerances may be relaxed in the spereitic case of the Orsay BSM. This derice is not sensitive to the longitudinal position of the waist within the length of a slit of typically 400 microns, and the depth of focus at the FFTB is of the order of $13_{y}^{*}=100 / \mathrm{m}$, allowing the waist to move around the nominal position without affecting the measurement of the minimm spot si\%e. A $2 \%$ increase in spot size corresponds to a waist movement of 20 microns for the nominal $3_{y}^{*}=100 \mu$. In the case of other spot size monitors such as the licpuid wires, we will have to noect these tight tolerances.

The FFTB power supplies have been specified arcording to these values. The goal for their stability is $0.001 \%$ or $10^{-5}$ of the full power for both long and short term stability. Some magnets clo not solicit the full power from the power supplies so this value will not be as good for the real current delivered. It is expected however that the tolerances presented here will be mot.

Finally some higher oreler effects are also triggered by strength errors of cuadrupoles within the CCS. The tolerances ${ }^{[56]}$ for these effects are at the loved of $\frac{\Delta k}{k} \leq 10^{-3}$ and therefore shomld not be a problem.

\section{j.j. Horizontal Sextupole Alignment.}

\section{5.j.1. Scxtupoles}

The sane (qualmpole effect (waist motion) appears when the beam is horizontally offset in a sextupole. The Hamiltonian of a sextupole in the presence of a horizontal risplacement is

$$
\begin{aligned}
H_{s} & =\frac{l_{s s}}{3 !}\left(\left(x+d_{x}\right)^{3}-3\left(x+\left(l_{x}\right) y^{2}\right)\right. \\
& =\frac{k_{s}}{3 !}\left(\ldots+3 l_{x} x^{2}-3 l_{x} y^{2}+\ldots\right)
\end{aligned}
$$

which shows a (1uadrupole aberration of the form

$$
H_{1}=\frac{k_{s} l_{x}}{2}\left(. r^{2}-y^{2}\right)
$$

The condition for the $2 \%$ ariterion is then

$$
d_{x} \leq \frac{1}{j k_{s} \operatorname{Max}\left(3_{x} \cdot 3_{y}\right)}
$$

The tolemaner fon horizontal sextupole alignment in the C'X is 3.5/1 while it is only $0.9 / 1$ 
for the C'C where the vertical 3 -function is very large (cf. figure 5.3 ).

\subsubsection{Orbit offset}

Similar to the dispersion case, a sextupole offset can be the consequence of an actual sextupole displacement or a quadrupole upstream steering the beam off-axis in the sextupole. The notion of multipliers applies also here with the reference being now at the sextupole.

$$
\left(d_{x}\right)_{s}=k_{q} R_{q \rightarrow s}^{12}\left(d_{x}\right)_{q}
$$

The tolerance on quadrupole alignment : agarding this effect is obtained by summing orer the subsecfuent sextupoles:

$$
\left(d_{x}\right)_{q} \leq \sum_{s>q} \frac{\left(d_{x}\right)_{s}}{k_{q} R_{q \rightarrow s}^{12}}
$$

Two sextupoles of the same pair have the same $d_{x}$ tolerance and are separated by a $-I$ transformation, therefore if the beam is off-axis in the first sextupole the effect will be cancelled by the equal and opposite displacement in the second sextupole of the pair. This means that quadrupole alignment within a sextupole pair is of special importance and the orbit jitter or a quadrupole clisplacement before entering the chromatic correction section is not crucial for this aberration ${ }^{*}$.

This effect is reftected in figure 5.3. The horizontal alignment tolerances of cquadrupoles within the C'CS are dominated by this effect. Notice that the contral cuadrupole in the chromatic correction section is especially ill-placed with regard to this effect since its position maximizes the multiplier to the second sextupole. This leads to very tight tolerances for these central culadrupoles: $0.7 \mu$ for $\mathrm{QN} 1$ in the $\mathrm{CCX}$ and $1.0 \mu$ for QM2 in the $\mathrm{CCY}$

\footnotetext{
* It is of importance for other aberrations like dispersion.
} 


\section{5.j.3. Brnding mangnets}

Finally another somere of orbit offset at the sextupoles are the fluctuations of the field, through power supply jitter, in bending magnets. Becausc of the length of these magnets and the fact that optical functions vary a lot across them, we define the multipliers as an arerage accoss the magnet; The $\overline{R_{12}}$ is the arerage value of the $R_{12}$ between the value at the entrance and the value at the exit of the bend. Several bending magnets connected in series to one power supply are treated as one large bend. The displacement induced at the sextupole,

$$
\left(d_{x}\right)_{s}=\Delta \theta \overline{R_{b \rightarrow s}^{12}}
$$

riclels the tolerance on power supply stability since $\theta \propto B$ :

$$
\frac{\Delta B}{B} \leq \frac{1}{5 k_{s} \overline{R_{12}} \theta \operatorname{Max}\left(\beta_{x}, \beta_{y}\right)_{s}}
$$

Notice that since, by design of the CCS, we have $\overline{R_{12}} \theta=211 . x$ at the sextupole, the preceding formula ran be rewritten, with $\xi_{x}=2 k_{s} \eta_{x} \beta_{x}$ and $\xi_{y}=2 k_{s} \eta_{x}$. $\xi_{y}$ the horizontal and vertical ('hromaticitics introduced by the sextupole pair:

$$
\frac{\Delta B}{B} \leq \frac{1}{5 \operatorname{Max}\left(\xi_{x}, \xi_{y}\right)}
$$

Here also only the bends between the sextupoles of a pair are significant for this effect. Field jitter of bending magnets outside these sections contribute to other aberrations such as rispersion. The stability on the power supplies for the bends has to be better than $3.310^{-5}$ for the C'CX and only $1.010^{-5}$ for the CCY. This last value corresponds to the goal set for the power supply stability at full power. It is also the tightest requirement for power supplies at the FFTB, even tighter than the tolerances set for the stabilicy of the final lenses.

Note finally that this cialculation accounts only for the geometric effect of the sextupoles. The change in bending angle also creates additional dispersion at the sextupole and therefore an adclitional chromatic kick. The change in dispersion from a bending field error is actually (cpual to the beam offset at the sextupole $\overline{R_{12}}$ d $1 \theta$. This chromatic effect is however smaller than the grometric effect by a factor $\bar{\delta}$, so that the tolerance is looser by a factor $1 / \bar{\delta}_{\text {rms }}$ or (1) 


\section{ifi. Skew Quadrupole.}

The roll, rotation aromel the longitndinal axis, of a culadrupole by an angle $\theta$ introdures a skew-cuadrupole component of strength $k_{s q}=k_{1 q}$ sin $2 \theta$ which rouples the horizontal and vertical planes. In the limit of small angles the Hamiltonian of the skew-cuadrupole aberration is

$$
H_{s q}=\frac{1}{2} k_{q} \sin 2 \theta(2 \cdot x y) \approx 2 k_{q} \theta x y
$$

giving one kick in each plane. However in the flat beam regime, which is the case for most final focus systems, i.e. with $\epsilon_{x} \gg \epsilon_{y}$, the effect of (oupling $x \rightarrow y$ is (lominant so we (an consider the vertical kick only. The tolerance criterion is

$$
\begin{gathered}
2 k_{q} \theta \sigma_{x} \leq \frac{1}{5} \sigma_{y^{\prime}} \\
\theta \leq \frac{1}{10 k_{q} \sqrt{3_{x}, \beta_{y}}} \sqrt{\frac{\epsilon_{y}}{\epsilon_{x}}}
\end{gathered}
$$

Except for the final cuadrupoles, all lenses in the line lawe tolerances better than 100 prad when taken individually. The RMS value however is only 40 furd. Note that the two high values for (QN1 and QN2 are not significant since the above formula was obtained in the approximation of small angles. However these two magnets are located at a multiple of $\pi$ in phase away from the IP and therefore their rotations does not affect the final spot size.

The final doublet has much tighter roll tolerances with a lowest for QC2 where the product $x_{x} y_{y}$ is very high. Sote that for a general final clomblet. it has been shown by John Irwin that the rotation tolerances of both lenses are the sane. In our case, with the split final lens (QX1-QC:1), this property is rerified if we consider this split final lens to be rane mique element. This tolerance is of the order of 10 prad. However for practical purposes the last three lonses can be considered to be one single rigid body, tied together by the stabilization table clesigned by KEK. Lsing this model, the rotation tolerance for the Q(')-QX1-QQ'1 grom? is 33 marl.

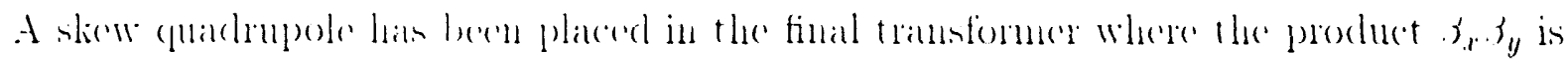

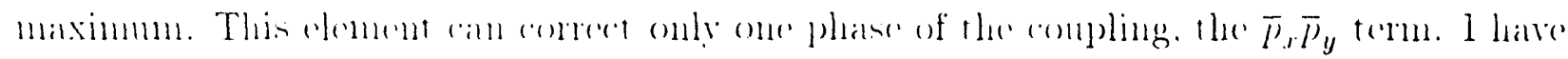




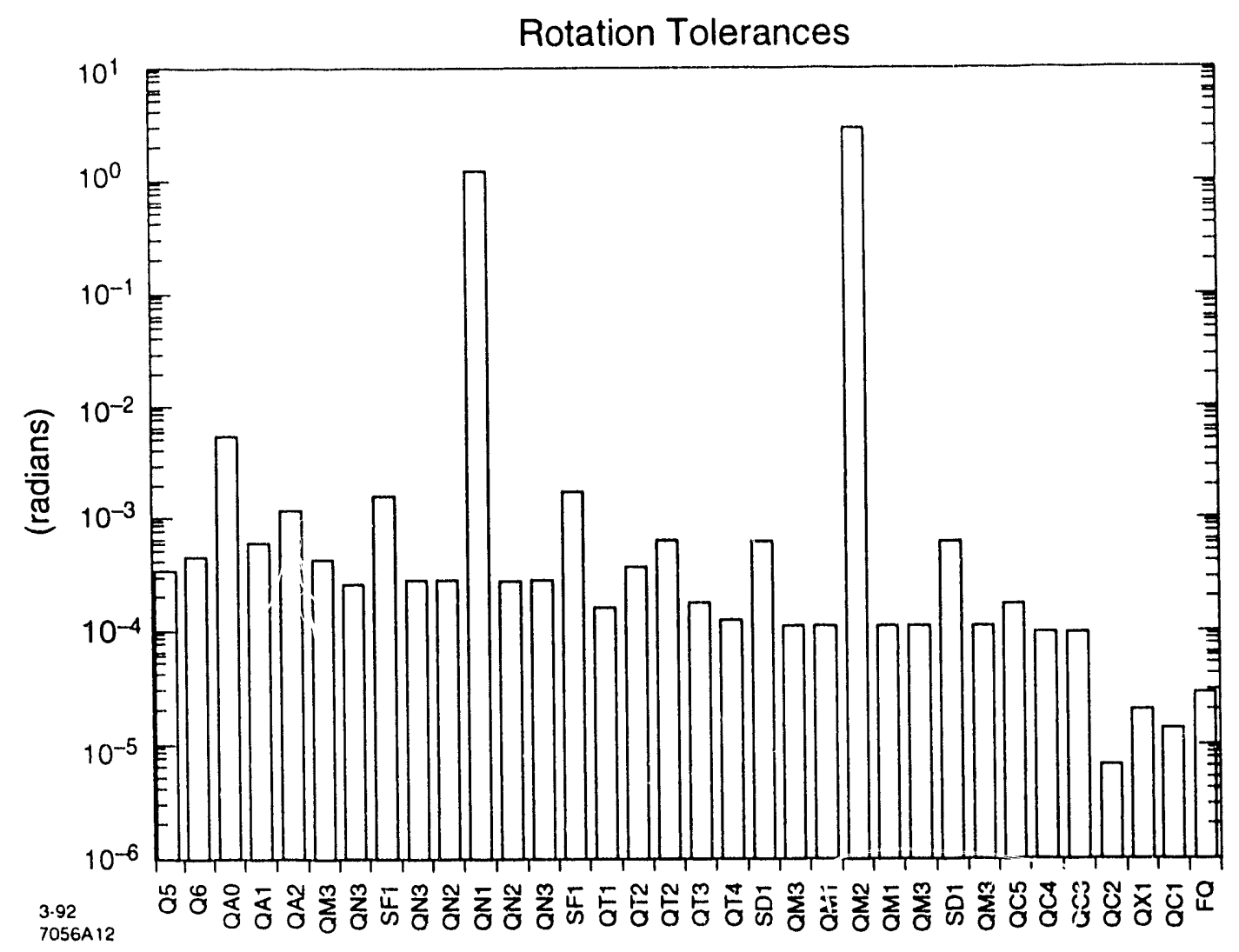

Figure 5.6. Quadrupole rotation (roll) tolerances. Note that the formula used here assumes a small angle approximation so the two peaks obtained for QN1 and QN2 are meaningless. These two magnets being $(n \pi)$ away in phase from the IP do not influence the size of the beam through this effect, which is the real meaning of these large values. For the sext npoles. the tolerances are estimiated for the geometric skew-sextupole effect.

argued already ${ }^{*}$ that due to the phase advance pattern at the FFTB, this term is the only one that can arise significantly in this line. Incoming coupling from the linac is corrected in the Beta Matching section using two skew quadrupoles.

Also on figure j.6 are the tolerances for sextupole rotation. The effect considered here is that of the geometrie skew sextupole. The tolerances are higher than those for the quadrupoles, at the level of one to two milliradians.

* cf. (diapter 4 . 


\section{‥ Vertical Sextupole Alignment.}

iт1. Sextupoles

The same coupling effect arises when the beam is vertically offiset in a sextupole. Similar to the nomal (quatrupole case this can be raused by a sextupole displacement, vertical stering firne a chardrupole offset or rlipole rotation inside the CCS. The Hamiltonian of a sextupele in the presenes of a vertical displacement is

$$
\begin{aligned}
H_{s} & =\frac{k_{s}}{3 !}\left(x^{3}-3 x\left(y+l_{y}\right)^{2}\right) \\
& =\frac{k_{s}}{3 !}\left(\ldots-6 d_{y} x y+\ldots\right)
\end{aligned}
$$

showing a skew-(puadrupole aberration of the form

$$
H_{s}=\frac{k_{s} d_{y}}{2} x y
$$

Zsing only the vertical lick again, the condition for the $2 \%$ criterion is then

$$
d_{y} \leq \frac{1}{5 k_{s} \sqrt{\beta_{x} \beta_{y}}} \sqrt{\frac{\epsilon_{y}}{\epsilon_{x}}}
$$

As shown on figne 5.4 , the tolerances are $3.5 \mathrm{fm}$ and $1.4 \mu \mathrm{m}$ for respectively $\mathrm{CCX}$ and C'C'.

The following table summarizes the sextupole alignment tolerances for both the horizontal and rertical displacements.

\begin{tabular}{|c|c|c|}
\hline & Horizontal & Vertical \\
\hline C'C'X $^{\prime}$ & $3.5 \mu m$ & $3.5 \mu m$ \\
\hline $\mathrm{C}^{\prime} \mathrm{C}^{\prime}$ & $0.9 \mu \mathrm{m}$ & $1.4 \mu \mathrm{m}$ \\
\hline
\end{tabular}

Table 5.1 Sextupole aingment tolerances at the FFTB. 


\section{T.2. () Hbit offset}

The same effect we have seen for dispersion and normal guadrupole tolerances appears here: a quadrupole offset upstream of the sextupoles can generate an orbit offset in the sextupoles leading to a skew-quadrupole effect. Similarly to the case of horizontal sextupole offset, this can only happen for quadrupoles located in the CCX or the CCY, within a sextupole pair. The efferts from the two sextupoles cancel each other for a quadrupole offset in the $\mathrm{BM}$ or $\mathrm{BX}$ sections.

T'he multipliers for quadrupole vertical alignment tolerances are

$$
\left(d_{y}\right)_{s}=k_{q} R_{q \rightarrow s}^{34}\left(d_{y}\right)_{q}
$$

Here also the two (puadrupoles at the miclpoint of the C'CS maximize these multipliers and this leads to tolelances of $4 \mu \mathrm{m}$ for CCX and only $0.3 \mu \mathrm{m}$ for the CCY. The latter is the tightest alignment tolerance at the FFTB. This quadrupole is physically located inside the Beam Switch Yard where the temperature is expected to be stable. Special care must however be given to the alignment of this critical lens. Some diagnostic and correction tools to control this skew (quadrupole effect are also being clesigned.

\subsubsection{Bending magnets}

The rotation of hending magnets within the $C^{\prime} C^{\prime} X$ and $C^{\prime} C^{\prime} Y$ also leads to an orbit offset in thr sextupoles. The multipliers for bending magnet rotation, defined as the ratio of the orbit offiset at the sextupoles to the dipole rotation angle $\Delta o$ leads to the following tolerances,

$$
\Delta \phi \leq \frac{1}{5 h_{s} \overline{R_{34}} \sqrt{\beta_{x} \beta_{y}} \theta} \sqrt{\frac{\epsilon_{y}}{\epsilon_{x}}}
$$

where $\overline{R_{34}}$ is the archage value of the $R_{34}$ across the bends talien separately.

The rotation tolerances are $\Delta \phi \leq 37$ prad for the CCX but only $\Delta \phi \leq 14$ prad for the $C^{\prime} C^{\prime}$. This is the tightest rotation tolerance at the FFTB. 


\subsection{Sextupole and Skew Sextupole.}

Thir tolerances on the sextupole and skew sextupole content of cuadrupoles are usually expressed as a ratio of the allowed sextupole or skew sextupole field romponent to the nominal (yuadrupole field taken at some reference point (usually $70 \%$ of the aperture of the magnet) $\left(B_{n . s} / B_{q}\right)_{a=a_{r}}$. One can also express this in terms of some equivalent sextupole strength $k_{n s}$ given hy

$$
\left(\frac{B_{n s}}{B_{q}}\right)_{a=a_{r}}=\frac{k_{n s} a_{r}}{2 k_{q}}
$$

The genemal sextupole Hamiltonian, neglecting chromatic effects as well as the effect of dispersion, $H_{n,}=\frac{k_{4}}{3 !}\left(x^{3}-3 r y^{2}\right)$, can give rise to three different kicks, two in the horizontal plane and one in the vertical plane. We first derive the $2 \%$ criterion condition for each kick.

- $x$ kick from the $x^{3}$ term $\Delta x^{\prime}=\frac{k_{n s}}{2} x^{2}$

$$
\begin{aligned}
& \frac{k_{n s}}{2}\left(\sqrt{2} \sigma_{x}^{2}\right) \leq \frac{1}{5} \sigma_{x^{\prime}} \\
& k_{n s} \leq \frac{\sqrt{2}}{5 \sigma_{x}: 3_{x}}=t_{x 1}
\end{aligned}
$$

- $x$ kick from the $x y^{2}$ term $\Delta x^{\prime}=\frac{-k_{u \varepsilon}}{2} y^{2}$

$$
\begin{gathered}
\frac{k_{n s}}{2}\left(\sqrt{2} \sigma_{y}^{2}\right) \leq \frac{1}{5} \sigma_{x^{\prime}} \\
k_{n s,} \leq \frac{\sqrt{2}}{5 \sigma_{y} \sqrt{\beta_{x} \beta_{y}}} \sqrt{\frac{\epsilon_{x}}{\epsilon_{y}}}=t_{r 2}
\end{gathered}
$$

- y kick from the r. $y^{2}$ torm $\Delta y^{\prime}=-k_{n s} \cdot x y$

$$
\begin{gathered}
k_{n s} \sigma_{x} \sigma_{y} \leq \frac{1}{5} \sigma_{y^{\prime}} \\
k_{n s s} \leq \frac{1}{5 \sigma_{x} \xi_{y}}=t_{y}
\end{gathered}
$$


For the compenmeling of these conditions, one notices that the offect of the at and y kicks are orthogonal in the semse that reye affect respectively the horizontal and rertical spot size: therefore we an take the minimum of the two conditions one in arch plane as the final expression for so xtmpolo field tolerances.

For the two conclitions related to the re kick. one can consider that they are separate aberrations affereting the same parameter and that their effects are therefore to be added in quadrature. The toleranec $t_{x}$ is then given by $1 / t_{*}^{2}=1 / t_{x 1}^{2}+1 / t_{x 2}^{2}$ and we finally have

$$
h_{n .} \leq \operatorname{Min}\left[\frac{\sqrt{2}}{5 \sigma_{x} \beta_{x} \sqrt{1+\sigma_{y}^{4} / \sigma_{x}^{4}}}: \frac{1}{5 \sigma_{x} \beta_{y}}\right]
$$

The same callenlation an be made for the skew-sextmpole component with the Hamiltonian $H_{s,}=\frac{k_{3}}{3 !}\left(3, r^{2} y-y^{3}\right)$. giving two vertical and one horizontal kicks, and yields

$$
l_{s, s} \leq \operatorname{Min}\left[\frac{\sqrt{2}}{5 \sigma_{y j} \beta_{y y} \sqrt{1+\sigma_{. x}^{4} / \sigma_{y, 4}^{4}}}: \frac{1}{5 \sigma_{y / r} \beta_{x}}\right]
$$

Althomgh these mumbers represent tight tolerances. the quadrupole magnets received at SLAC from INP (Aorosibirsk) have all met or execeded these tolerances, according to the magnetic moasmenements anvied ont at INP and SL.AC:

The final (1nadruy)oles represent an additional challenge with even tighter tolerances, esperally for $\left(2 C^{2} 2\right.$. Rerent results from KEK have shown that it is possible to meet and exered these toleranees by using trime windings: In order to suppress the drift of the quadrupole renter with magned exitation. the use of trim windings hats bern suggested by Nakayama at KEK. The revitation of the trims being proportional to that of the main eoils. A side effect of this methen. observer on ihe prototype magnet, is the cancellation of the sextupole and skew sextmpole temo in these final guadrupoles to a level better than the recuired tolerances.

Arlitionally we have proposed a global correction of these terms by use of two sextupole and two skin-sextupole correctors in the Final Transformer. These four small magnets wonkl allow us to tume ont the four tems appearing in the wo Hamiltonians quoted above. If the trin windiuge of the final quadrupoles to be installed in the tumnel also supperess the

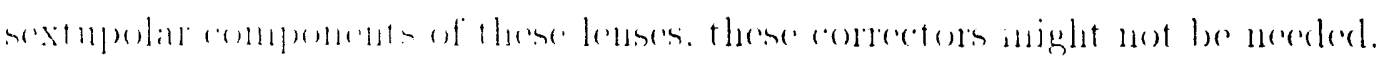




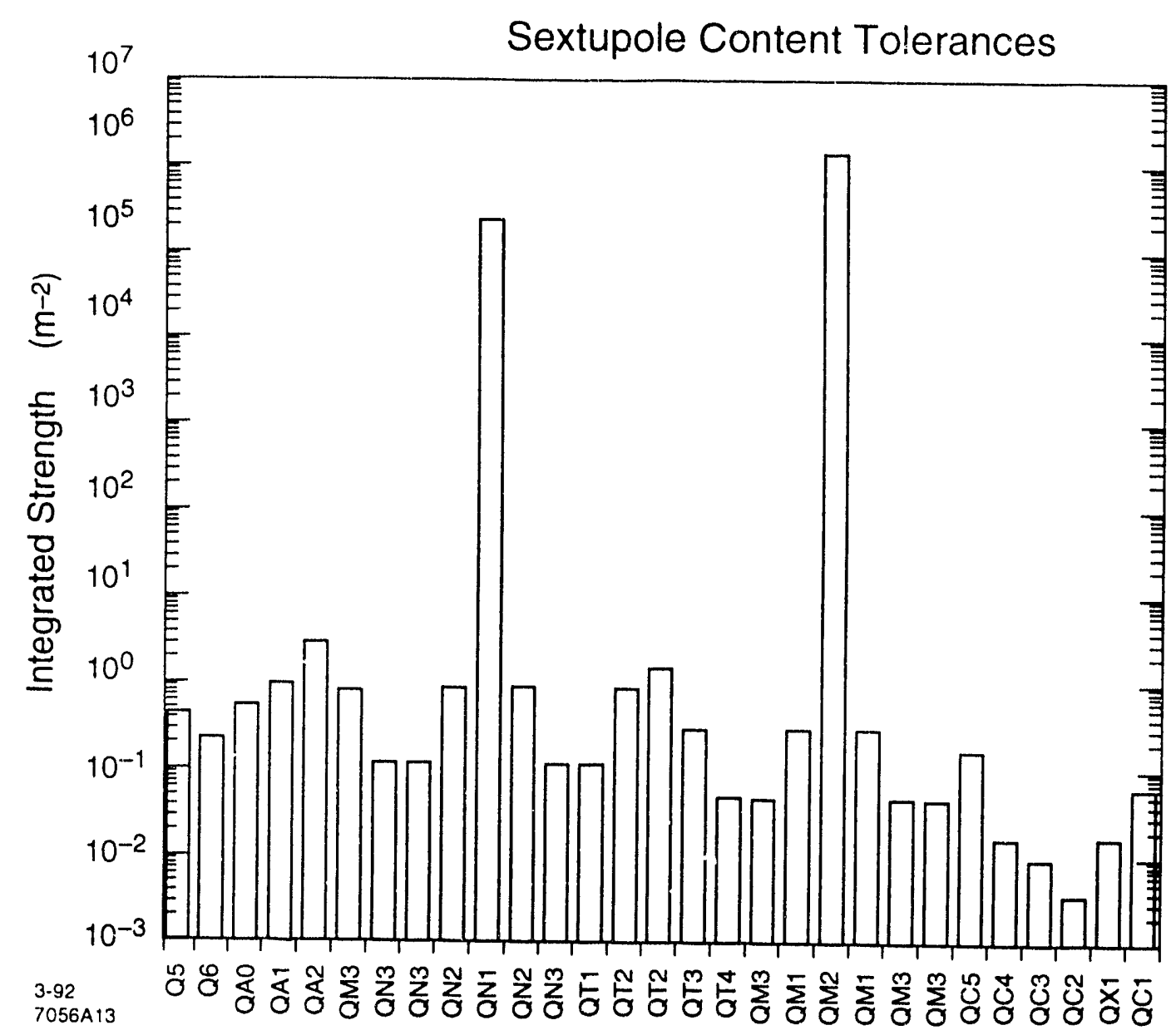

Figure 5.7. Sextupole and skew sextupole harmonic tolerances in cuadrupoles. The worst of the two components is shown here. The tolerance is expresserl in terms of ecpuvalent integrated strength. The main sextupoles at the FFTR have an integrated strength of alsout $20 \mathrm{~m}^{-2}$.

\section{j.9. CONClusion}

I have shown in this chapter how we obtained stability tolerances for the FFTB. The use of Lie alged ra based methorels is here limited to the expression of the kick in most cases. The sturly of sextupolar tolerances however is one where the combination of the different terms is greatly simplificd by the nse of the Hamiltonian formulation of the soure of the effects. For all these calculations hewever it would be possible to derive the same results without Lie algebra formulation. However it is my experience that Lie alg(b)ra based methods, as presented in the previons chapters, provide here also a very convenient and clear framework for this tripe of sturlios.

For all practical purposes the stability tolerances are very tight at the FFTB. The SLC final focus hats lowser tolcrances by typically an order of magnitude when compared to the 
PFTB. Studies ${ }^{[2]}$ hate shown that tolerances for a final focus system for a next generation linear collider. typically a job Ger per beam machine, will be tighter by another order of magnitude.

Of particular inportance in the results of this chapter is the notion of lattice multipliers. The optics of final focus systems typically altemate very high and very low is.functions. This loads maturally to high lattice multipliers. It is not always possible to limit the values of these ; 3 -functions but it should be possible, at least very desirable, to suppress the ill-placed clements such as the two central quadrupoles located at the symmetry points of both CCS. The position tolerances of these two elements are the tightest of all at the FFTB.

Another important result is the level of stability required for the bending magnets, esperially in the C'CY. They hold the tightest tolerances for strength and roll stability, aside fiom the final lense's of course.

Much is to be leant fiom the tuning and operation of the FFTB and this is especially true for toleranees. Whother one an achieve the stability levels required here is one issue. The other (fuestion is, if the stability tolerances camnot be achieved, do we have a mechanism by which onc "an go aromud the limitations by using simple foedback systems and careful tuning statcegics for examplo? We believe that it is possible to relax some initial tolerances at the FFTB bump-based (quadrupole tuming schemes. Global correctors such as the dispersion suppression and the sextupole corrector magnets are other teciniques that are being investigated at the FFTB. 


\section{Conclusion}

The Final Focus: Trst Beam is now well underway and the first beam should be delivered by spring 1993. This will be the start of a very interesting period on the way to future linear colliders. We will test on a full scale machine the hardware we think is critical for the NLC. The beam position monitors, magnet movers, wire scamners and a number of stabilization and monitoring devices will be commissioned. The software side will also be scrutinized with the correction and tuning techniques that are now being developed at SLAC. These are (especially important for their potential impact on the tolerances. The optics itself will be probed of course, and once the tuning and corrections are applied, the measurements terhnicunes will be commissioned: the Orsay-Beam Size Monitor, but also the liquid wires and the Laser-Compton device. I think we can expect a great harvest of information with the FFTB and coltainly loain even more than we did with the SLC final focus. Parallel to this clesign work on NLC final focus lattices is being pursued.

The FFTB is also minge in the sense that it is the first beamline for which Lie algebra biscel technigues were used from the design up to the derivation of correction and tuning procerlures. They have certainly proven helpful in all the areas of the work. The mechanisms of cancellation and the generation of new higher order aberrations is now very well understood. The faret that the list of aberrations at the FFTB is closed at fifth order has bren shorn using these tools. Finally the tolerances have bern analyzed with those same methorls and aldearly some irleas have emerged in order to reduce some of them. Part of the problem has bern inlentified as roming from the first onder design of the line. Work is mulcrway in different laboratories to confirm this. Some ideas have also been proposed to resign ways to cope with others, or find technicues for monitoring the stability of the line with a precision better than the tolerances. Quadrupole tuming at the FFTB is one of these technicpues that uses bumps to precisely test the local lattice and detect a strength error in cuadrupoles with a precision better than the tolerances. The potential consequences on the spreafication of tolenances for future machines conld be very important; The relaxation of most lolerances by a farefor two, and possibly as high as tem, is of great significance at the very tight levels we deal with. 


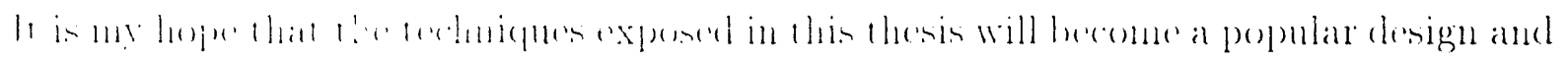

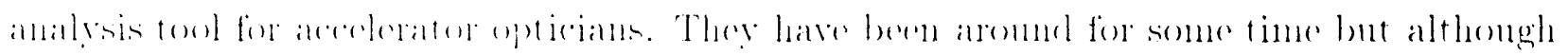

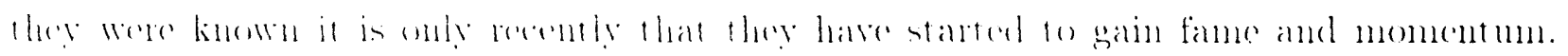

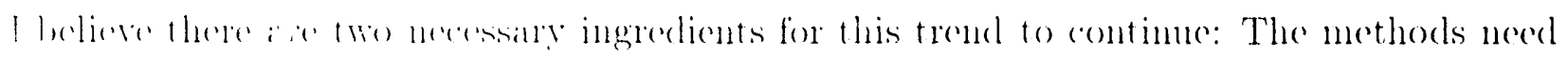
to he mose withely phlicized and explained and there is a nerel for a computer based tool. The first part is mostly a task of olucation and explanation. Although they are not very difficmlt in their mathematical basis as shown in this thesis, the loarning curve for these uew methods is fairly stere for neweomers and especially for people who have been using the matrix formalism for some time. It is a totally different way of seceng the basic same

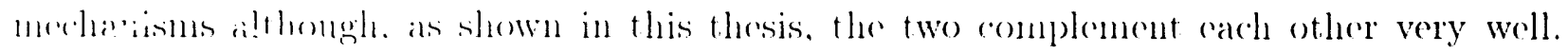
Explantitions and (exmples still need to be provided and this thesis is one attempt at this.

The of laer part repuired for the wide aceeptanee and use of these methods is the ability to use a emputing foul that will free the usei of the sometimes tedions calculations involved. Since the mothods presenterl are so much oriented towards analytical results a cole should be designed that prescres that option and allows analytical studies of a beamline as much

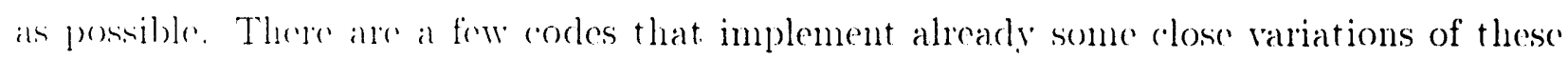

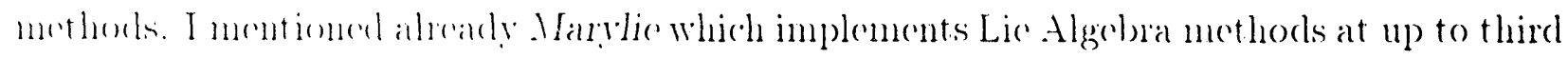

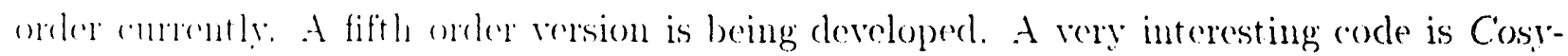
Infinity writtru by . М. Br\%. Although it implements only. Differential Algebra methods, it has berne curisioned for some time to adapt it for onr technicpues. The originality of this colde is that it prevides the usen with a meta-language that can be used to literally program the phrsics. That in the uno is no longer constrained to using the physics "anned" by the programmer in a preset command: on the contrary, all the variables are accessible to the user who (an drfine his own algonithus. Following a slightly differeni path and using the new,

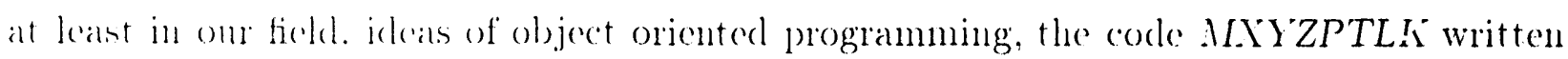
1 2 L. Michelotti at Fornilal, uses $C++$ as the programming language. The software is now

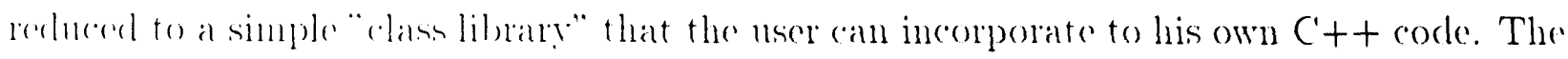

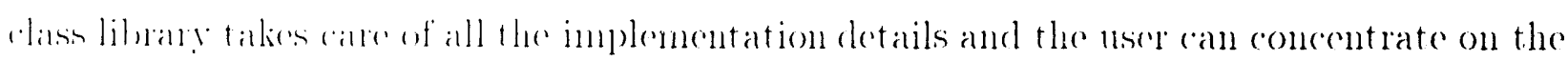
phisicis.

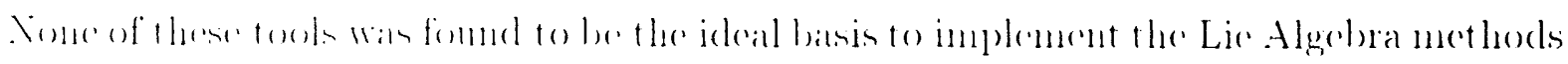

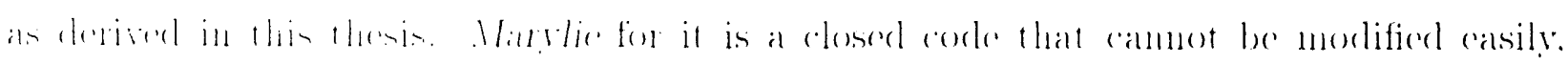

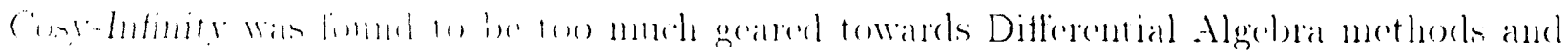


MXYZPTLK was not tesed but $C^{+}+$is known to hare a rather stecp loarning curve. The last direetion of investigation to be mentioned, which I believe stands the best chance to provide us with the needed tool is Mathematica. ${ }^{[33]}$ This cole was designed to perform analytical as woll as muncrical calculations on computers and is much in use worldwide. This ability of analytical manipulation together with a strong object oriented programming (nviromment, the possibility for the use to define his own programs on top of the parkage, and the munde desimalse feature of interactivity makes it the icloal candidate for these studies. At SLAC, the effort is led in this direction by J. Irwin and N. Walker. Some basic opties packiage is already dereloped and a Lie Algebra tool should soon come out. On top of being a good tool for accelerator physies analysis, this should also be the idcal support for newcomers to explore these technicues through tutorials.

There is one areat that has been mentioned in the first elatpter of this thesis and is not

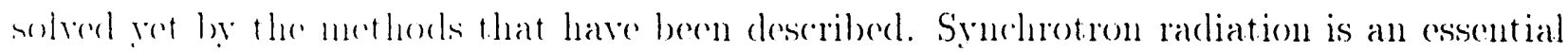
meress in final forms sustems since it is the somer of the main limiting terms. The stochastic: nature of the process malies it impossible to rescribe it through a Hamiltonian formulation. The only way at mesent to check for this effect is, except for analytical calculations as shown in appendix A. the nse of tracking methods. Most tracking codes now implement clifferent simmlations of the synchrotron radiation process. But we are back here to the "hlind" approarch of clesigning a system and ehecking a posteriori its valiclity. This area is one where somereffort and new ideas are needed.

After the exeollent work of Dragt and the big step madr mole" the impulse of Joln Irwin.

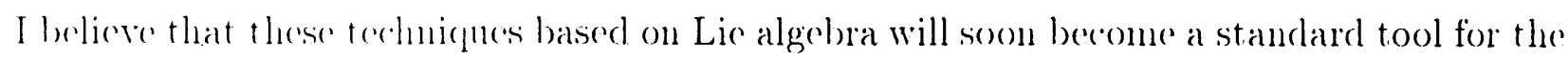
acerelerator physicist. There is howerer still a lot of room for improvements and new ideas. 


\title{
Appendix A
}

\section{Chromatic effects due to synchrotron radiation at the FFTB}

\author{
INTRODUCTION
}

I review here two effects induced by synchrotron radiation and their consequences on the final spot size at the Final Focus Test Beam. The first one is the chromaticity unbalance generated in a final focus system by the energy loss in bending magnets between the sextupoles and final cuadrupoles. The second more general effect is the emittance growth from radiation fluctuations. The latter has already been studied ${ }^{[52]}$ by Matthew Sands and I will use his results to derive a somewhat simpler and more practical formulation of this effect. An application to the case of the FFTB is given in both casces.

\section{Chromaticity UnBalance}

In a final focus system the chromaticity introduced by the very strong final lenses is canrelled at the interaction point by placing sextupoles in a dispersive region giving a chromatic kirk which can be made equal and opposite to that of the final lenses.

Howerer one neerls some bending magnets inside and after the chromatic correction section to manipulate the dispersion. In these bending magnets, the particles lose energy through syuchotron radiation; the energy of the particle in the sextupoles is slightly different from its chergy when it reaches the final quadrupoles. As a result the chromatic kicks do not balance carch of here exactly and there is an increase in the spot size at the focal point.

Katsmuolun Oide has already estimated this effect ${ }^{[5]}$ but I give here a more extended dorivation of it. 
The chomatie kick given by the final quadrupoles is given by $\Delta y^{\prime}=h_{q} \bar{\delta} y$ and is balanced by the elnomatic lick at the sextupole, moler the hypothesis that there is only one sextmpole rorecting all the ehementicity, $\Delta y^{\prime}=k_{s} \eta \bar{\delta}_{y}$. If the particle loses energy ${ }^{\star}$ between the two we have $\bar{\zeta} \rightarrow \bar{\delta}-\frac{d u}{E_{1}}$ at the final (quadrupole and the net ehromatie kick is

$$
\Delta y^{\prime}=\operatorname{liq}_{q} \frac{d \|}{E_{0}}, y
$$

where $E_{0}$ is the cuergy of the beam and du is the energy lost through radiation. The change in position at the IP is writen, with $y_{q}$ the particle position at the final quadrupole:

$$
\Delta y^{*}=k_{q} \sqrt{\beta_{q} \beta^{*}} \frac{d u}{E_{0}} y_{q}
$$

and the increase in spot size at the IP is generally witten

$$
\Delta \sigma_{y}^{*}=\sqrt{\left\langle\Delta y^{* 2}\right\rangle-\left\langle\Delta y^{*}\right\rangle^{2}}
$$

Because the two valiables $y_{q}$ and du are not correlated in first approximation as there is no design vertical dispersion. and since this effect does not change the centroid of the beam $\left\langle y_{q}\right\rangle$ which remains zero we have

$$
\left\langle\Delta y^{*}\right\rangle \times\langle d u\rangle\left\langle y_{q}\right\rangle=0
$$

alurl

$$
\begin{aligned}
\left\langle\Delta_{y^{* 2}}\right\rangle & =k_{q}^{2} 3_{q} 3^{*} \frac{1}{E_{0}^{2}}\left\langle d u^{2} y_{q}^{2}\right\rangle \\
& =k_{q}^{2}, 3_{q} \beta^{*} \frac{1}{E_{0}^{2}}\left\langle d u^{2}\right\rangle\left\langle y_{q}^{2}\right\rangle \\
& =k_{q}^{2} i_{q} 3^{*} \frac{1}{E_{0}^{2}}\left\langle d u^{2}\right\rangle \sigma_{y_{q}}^{2} \\
& =k_{q}^{2} \beta_{q}^{2} \frac{1}{E_{0}^{2}} \sigma_{y}^{* 2}\left\langle d u^{2}\right\rangle
\end{aligned}
$$

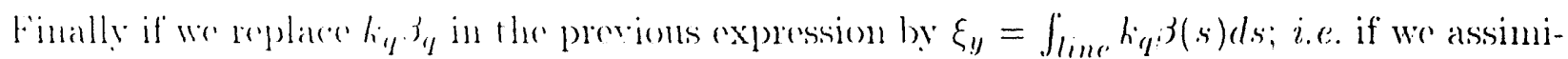

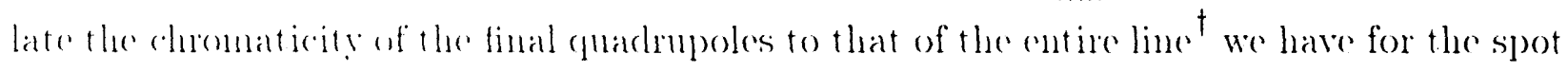
size increase at the foral point:

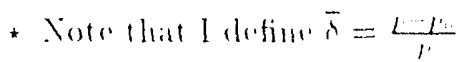

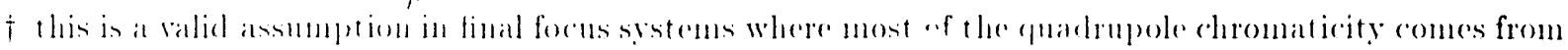

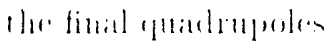




$$
\frac{\Delta \sigma_{y}^{* 2}}{\sigma_{y}^{* 2}}=\frac{\xi_{y}^{2}}{E_{0}^{2}}\left\langle d u^{2}\right\rangle
$$

Energy loss considerations

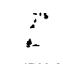

There are two terms in the energy loss through syuchrotron racliation. The first one is the so-called "chassical or average energy loss" $\langle d u\rangle$; the second one is a consecunence of the (quantum nature of syuchetron radiation and leads to an increase in the energy speread and (quantum excitation of oscillations: it is stochastic in nature.

The arerage energy loss in our case has for a consequence that the energy of the beam at the entrance of the final cuadrupoles is slightly lower than the design value, the cuadrupole then focuses this beam a little upstream of the nominal IP, henec some "spot size increase" at the IP'. This effere can be compensated for by retuning the cuaclrupoles for the new beam chrergy:

Also the sextupoles need retuning since the chromatic kick now given by the quadrupoles: is smaller a the bean energy and the quadrupole strength are now lower than the design value. This efferet will be automatically corrected for in the sextupole strength optimization procedure during the tuning of the tinal spot size.

Me sere here that the arerage energy loss cau be compensated for and should therefore be remered firom the present calculation. The stochastic component of synchrotron radiation

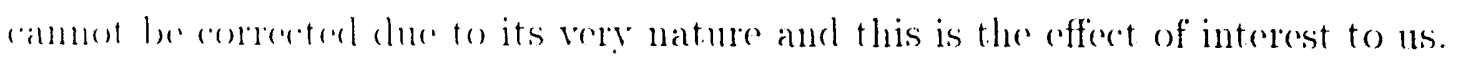

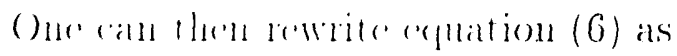

$$
\frac{\Delta \sigma_{y}^{* 2}}{\sigma_{y}^{* 2}}=\frac{\xi_{y}^{2}}{E_{0}^{2}}\left(\sigma_{d u}^{2}+\langle d u\rangle^{2}\right)
$$

and droppling the second trm which is correctable,

$$
\frac{\Delta \sigma_{y}^{* 2}}{\sigma_{y}^{* 2}}=\frac{\xi_{y}^{2}}{E_{0}^{2}} \sigma_{d u}^{2}
$$

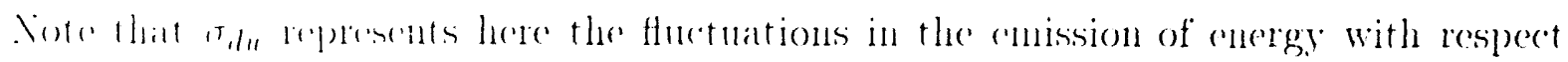

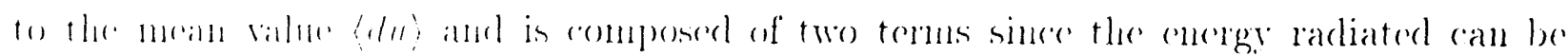




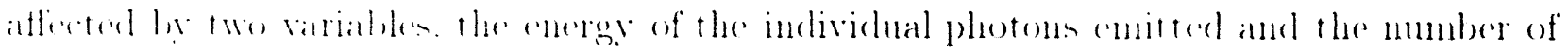
photons rnitferl. Eateh emission of a photon being independent of the others the first term is simply the guadratic smm over the average number of photons of the spread in the energy distribution of the photons. The second tem is characterized by the spread in the number of emitted pheton times the average photon anergy: Since the mumber of emitted photon in clistributed according to a Poisson distribution with mean . V. the mean square of the distribution is also $x$

$$
\begin{aligned}
\sigma_{d u}^{2} & =\left(\frac{l b}{c} N\right) \sigma_{u}^{2}+\langle u\rangle^{2} \frac{l_{b}}{c} \sigma_{n}^{2} \\
& =\frac{l}{c} N\left(\sigma_{u}^{2}+\langle u\rangle^{2}\right) \\
& =\frac{l b}{c} N\left\langle u^{2}\right\rangle
\end{aligned}
$$

ancl finally:

$$
\sigma_{d u}^{2}=\frac{55}{24 \sqrt{3}} \frac{\theta^{3}}{l_{b}^{2}} a_{i}^{5} r_{e} \lambda_{e} E_{0}^{2}
$$

Result

The final result for the increase of the spot size at the Interaction Point is then

$$
\frac{\Delta \sigma_{y}^{* 2}}{\sigma_{y}^{* 2}}=\frac{55}{2+\sqrt{3}} r_{e} \lambda_{e} \xi_{y}^{2}-s^{5} \frac{\theta^{3}}{l_{l}^{2}}
$$

IVith a chromaticity $\xi_{y} \sim 17 \times 10^{3}$. a beam energy of 50 Gel giving $; \sim 10^{5}$ and a lunding magnet with length $l_{b}=5.2 \mathrm{~m}$ and bending angle $\theta=7.4$ mrad one gets $\frac{\Delta \sigma_{y}^{* 2}}{\sigma_{y}^{*}}=$ ().06. (omesponding to an increase in the spot size of $\frac{\Delta \sigma_{y}^{*}}{\sigma_{y}^{*}}=1-\sqrt{1+.06} \sim 3 . \%$

THE WHOLE LINE

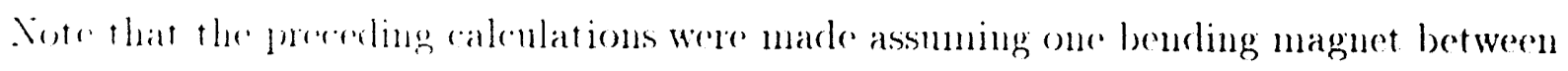
a single sextupole and the final quadrupoles. At the FFTB we have two pairs of sextupoles and six (grompon of ; bending magnets arranged in the following way: 


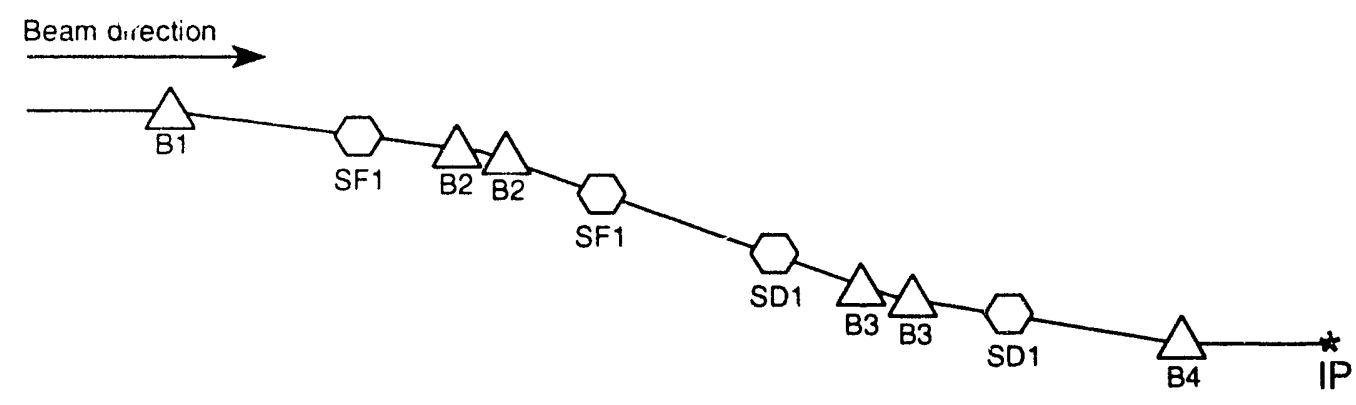

Figure 7.1. The disposition of bending magnets at the FFTB.

It is clear that magnet $B_{1}$ does not contribute to the process we describe since the total chromaticity of the line from $B_{1}$ to the interaction point is zero by design. Magnet $B_{4}$ contributes the most to this effect as it affects the full chromaticity of the final cuadrupoles. Most of the vertical chromaticity is corrected in the $\mathrm{C}^{\prime} \mathrm{C}^{\prime}$ and even if we assume tha' $10 \%$ of it is corrected by the CCX. the effect on $\frac{\Delta \sigma^{2}}{\sigma^{2}}$ is down to the $1 \%$ level due to the square of the chromatieity appearing in the final formula. Assuming then that the whole chromaticity of the line is corrected in the $\mathrm{CCY}^{\circ}$ (we neglect the $\mathrm{CCX}$ altogether), each of $B_{3}$ sees only half of the total rhromaticity. contributing only a factor one-fourth to the total result. For the FFTB line we therefore !rave

$$
\begin{aligned}
\frac{\Delta \sigma_{y}^{* 2}}{\sigma_{y}^{* 2}} & =\left(1+\frac{1}{4}+\frac{1}{4}\right) \frac{55}{24 \sqrt{3}} r_{r} \lambda_{e} \xi_{y}^{2}, j \frac{\theta^{3}}{l_{b}^{2}} \\
& =\frac{55}{16 \sqrt{3}} r_{c} \lambda_{c} \xi_{y y}^{2} \gamma^{5} \frac{\theta^{3}}{l_{b}^{2}}
\end{aligned}
$$

Finally for the whole FFTB line we haw

$$
\frac{\Delta \sigma_{y}^{* 2}}{\sigma_{y}^{* 2}}=0.09
$$

(orresponding to

$$
\frac{\Delta \sigma_{y}^{*}}{\sigma_{y}^{*}} \sim 4.5 \%
$$

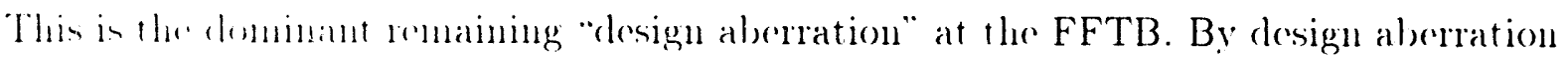

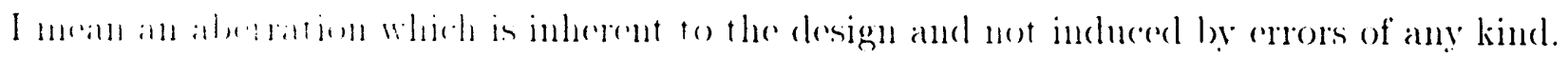

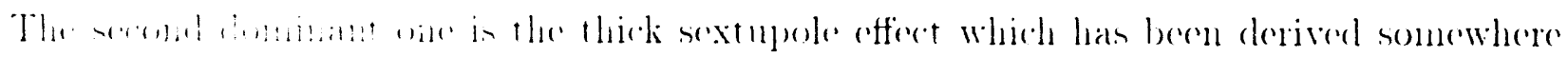
Hs. 


\section{EMITtance grower}

Introduction and conditions

In this section I rely on the results developed by Sands in reference [52] and I use them withent funther prost or explanation. Howerer I will recall here the three conditions under which those results were oldained.

- the transport system is linear.

This is essential to the method since a linear system allows one to apply a solution superposition principle: namely the displacement of the trajectory as a consequence of the emission of a $110 \mathrm{mber}$ of photous is just the sum of the individual displacements each emission would generate by itself. Non linear lenses complicate the process of adding the displacements at the and of the line. Although the FFTB is intrinsically non-lincar (strong sextupoles are Jresent in the lattice and the chromaticity of the final quadrupoles is very important) I will assmme for the moment that this first condition is verified.

- there is no coupling in the optics

This conclition simplifies the treatment of the problem by assuming that there exists everywhere in the line a miclplane symmetry. Then the emittance growth takes place only in the lorizontal planc. leaving the vertical emittance untouched (if we neglect the energy spread increase which affects both planes of course). This condition is verified in the case of the FFTB design in the absence of errors.

- the radution effects can be described by their characteristics on the central design trajectory.

Tlie main nuplication for the FFTB is that we neglect the radiation in quadrupoles and other lonses since the central trajortory. On-axis in those lenses assuming that we compensate this trajertory for the arrage curergy loss along the line, sees no field hence experiences no maliation. The catse of radiation in cuadrupoles and the resulting limitation on the beam size at the foral point has bern studied ${ }^{[5,1}$ for final focus systems by Katsunobu Oirle. Also the waik focming offeret in bending magnets is ignored. 
Resules and "residnal dispersion"

Sancls calculates the contribution to the second moments of the beam distribution in respectively equations (10), (11) and (12) in SLAC/AP-4T, where $C_{2}$ is a constant $C_{2}=$ $\frac{55}{24 \sqrt{3}} \frac{r \cdot f_{i c}}{\left(m c^{2}\right)^{6}}=4.13 \times 10^{-11} \mathrm{~m}^{2}\left(\mathrm{GeV}^{r}\right)^{-5}$.

$$
\begin{aligned}
\left\langle x^{2}\right\rangle & =C_{2} \int_{0}^{L} G^{3} E^{5} D^{2} d s=\frac{C_{2} E_{0}^{5}}{\rho^{3}} \int_{0}^{l_{b}} D^{2}(s) d s \\
\left\langle x^{\prime 2}\right\rangle & =C_{2} \int_{0}^{L} G^{3} E^{5} D^{\dagger^{2}} d s=\frac{C_{2} E_{0}^{5}}{\rho^{3}} \int_{0}^{l_{b}} D^{\dagger^{2}}(s) d s \\
\left\langle. x^{\prime}\right\rangle & =C_{2} \int_{0}^{L} G^{3} E^{5} D D^{\dagger} d s=\frac{C_{2} E_{0}^{5}}{\rho^{3}} \int_{0}^{l_{b}} D(s) D^{\dagger}(s) d s
\end{aligned}
$$

The main assmumption used to get the final form in the above equations are that the energy $E(s)$ and therefore the local trajectory curvature $G(s)=1 / \rho(s)$ (in a uniform bending magnet) do not change significantly along the path of the clectron. This is actually equivalent to the third condition of Sands approximating the characteristics of the radiation to that of an ideal particle on the central trajectory. The integrals have also been written assuming one bending manget of length $l_{b}$ followed by a region where $1 / \rho$ is uniformly zero.

The functions $D(s)$ and $D^{\dagger}(s)$ are the "residual dispersion" functions, i.e. the dispersion function from point $s$ in the bend to the end of the line, and its derivative. In TRANSPORT notation, they are the $R_{16}$ and $R_{26}$ terms calculated for the partial transport system from s to the end of the line. End of the line here refers to some arbitrary point where we want the emittance growth calculated. In the case of the FFTB this point is the final focal point (IP).

To calculate these second moments, Sands expresses the residual dispersion functions in terms of the betatem and off-energy functions $(3(s)$ and $n(s))$ as well as the phase advance along the line. I find it nore convenient to use the following argument using only the knowledge of the optics in terms of linear matrices.

I (an split the partial transport system (from point $s$ to the IP) into two parts. The first one is from point s inside the bending magnet (the origin $s=0$ is at the entrance) to the 


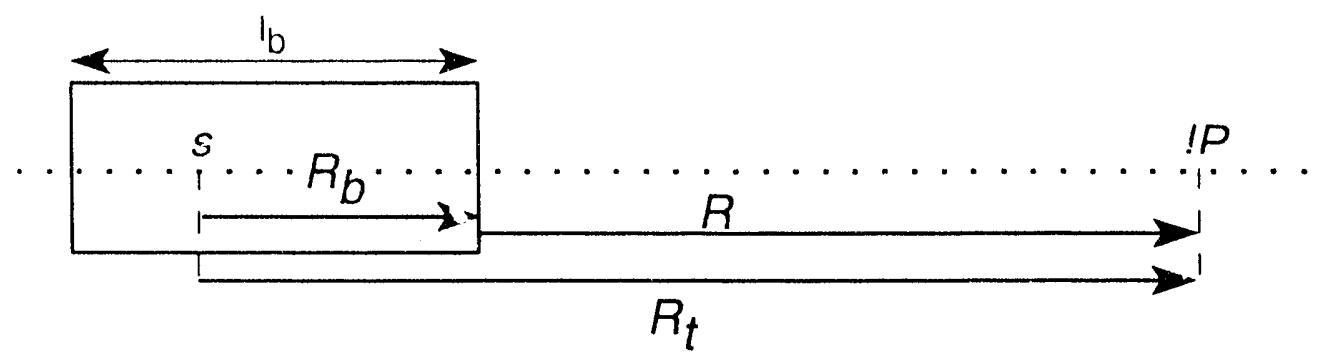

Figure 7.1. The trausport system used for the calculation of emirtance growth created by at single bend along with the notations used in the text.

enel of the magned and the serenel part is from the end of the bending magnet to the end of the line as represented on the figme below.

Let $R_{b}$ and $R$ be the respective first order transport matriees fo t aese two parts and $R_{t}$ be the first order matrix for the whole line from point s to the end so that we have $R_{t}=R . R_{b}$ ilicl

$$
\begin{aligned}
D(s) & =\left(R_{t}\right)_{10}=R_{11}\left(R_{b}\right)_{16}+R_{12}\left(R_{b}\right)_{26}+R_{10} \\
D^{\dagger}(s) & =\left(R_{t}\right)_{20}=R_{21}\left(R_{b}\right)_{10}+R_{22}\left(R_{b}\right)_{26}+R_{26}
\end{aligned}
$$

Now we assume that we know the design of the line to first order and therefore the $R_{i j}$ are known. We also know that. upon the assumption that the energy is not varying too much when the particle enits a photon and therefore $\rho$ does not change along the magnet, the clispersion finctions hare the following expression inside the bending magnet of length 1, :

$$
\left(R_{b}\right)_{1 C}=\frac{1}{2} \frac{\left(l_{b}-s\right)^{2}}{\rho} \quad \text { and } \quad\left(R_{b}\right)_{26}=\frac{\left(l_{b}-s\right)}{\rho}
$$

It in llen trivial to ant ont the integrals in equation (15). Mo have olstained here the

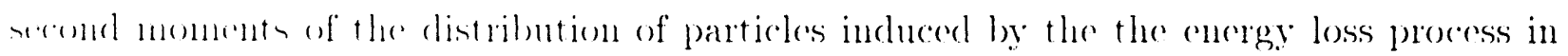

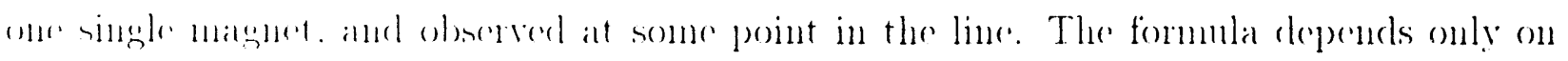

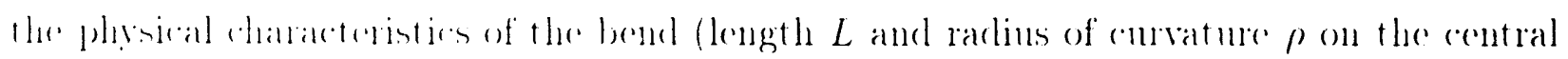

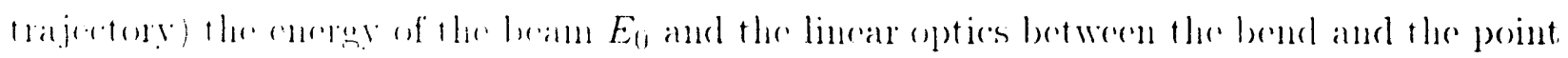


of observation ( $R$ matrix):

$$
\begin{aligned}
\left\langle r^{2}\right\rangle & =\frac{C_{2} E_{0}^{5}}{\rho^{5}} L\left[\frac{L^{4} R_{11}^{2}}{20}+\frac{L^{3} R_{11} R_{12}}{4}+\frac{L^{2} R_{12}^{2}}{3}+\frac{L^{2} R_{11} R_{16} \rho}{3}\right. \\
& \left.+L R_{12} R_{16} \rho+R_{16}^{2} \rho^{2}\right] \\
\left\langle r^{\prime 2}\right\rangle & =\frac{C_{2} E_{0}^{5}}{\rho^{5}} L\left[\frac{L^{4} R_{21}^{2}}{20}+\frac{L^{3} R_{21} R_{22}}{4}+\frac{L^{2} R_{22}^{2}}{3}+\frac{L^{2} I_{21} R_{26} \rho}{3}\right. \\
& \left.+L R_{22} R_{26} \rho+R_{26}^{2} \rho^{2}\right] \\
\left\langle x x^{\prime}\right\rangle & =\frac{C_{2} E_{0}^{5}}{\rho^{5}} L\left[\frac{L^{4} R_{11} R_{21}}{20}+\frac{L^{3} R_{12} R_{21}}{8}+\frac{L^{3} R_{11} R_{22}}{8}+\frac{L^{2} R_{12} R_{22}}{3}\right. \\
& \left.+\frac{L^{2} R_{21} R_{16} \rho}{6}+\frac{L^{2} R_{11} R_{26} \rho}{6}+\frac{L R_{22} R_{16} \rho}{2}+\frac{L R_{12} R_{26} \rho}{2}+R_{16} R_{26} \rho^{2}\right]
\end{aligned}
$$

Enittance growth

The calculation of the emittance growth from these formulas is justified by Sands in the definition of the RMS emittance as the three quantities $\left\langle x^{2}\right\rangle,\left\langle x^{\prime 2}\right\rangle$ and $\left\langle x x^{\prime}\right\rangle$ combined according to

$$
\epsilon_{x}^{2}=\left\langle x^{2}\right\rangle\left\langle x^{\prime 2}\right\rangle-\left\langle x x^{\prime}\right\rangle
$$

The folding of two RMS emittances $\epsilon_{a}$ and $\epsilon_{b}$, under the assumption that the transport system is linear, is giren by the addition in quadrature of the indlividual elements defining the enittance:

$$
\begin{aligned}
\left\langle x^{2}\right\rangle_{f} & =\left\langle x^{2}\right\rangle_{a}+\left\langle x^{2}\right\rangle_{b} \\
\left\langle x^{\prime 2}\right\rangle_{f} & =\left\langle x^{\prime 2}\right\rangle_{a}+\left\langle x^{\prime 2}\right\rangle_{b} \\
\left\langle x x^{\prime}\right\rangle_{f} & =\left\langle x x^{\prime}\right\rangle_{a}+\left\langle x x^{\prime}\right\rangle_{b}
\end{aligned}
$$

Finally we get for the total line the following formula defining the emittance increase, where the of sulseseript denotes the nominal enittance and the tsulseripts the tot al enittance: 


$$
\begin{aligned}
& \left\langle x^{2}\right\rangle_{t}=\left\langle x^{2}\right\rangle_{0}+\sum_{n}\left\langle x^{2}\right\rangle_{n} \\
& \left\langle\cdot r^{\prime 2}\right\rangle_{t}=\left\langle r^{\prime 2}\right\rangle_{0}+\sum_{n}\left\langle x^{\prime 2}\right\rangle_{n} \\
& \left\langle\cdot r r^{\prime}\right\rangle_{t}=\left\langle\cdot r r^{\prime}\right\rangle_{0}+\sum_{n}\left\langle\cdot r r^{\prime}\right\rangle_{n}
\end{aligned}
$$

with the $\left\langle x^{2}\right\rangle_{n},\left\langle.^{\prime{ }^{2}}\right\rangle_{n}$ and $\left\langle\cdot x^{\prime}\right\rangle_{n}$ defined in equation (18).

Note that with the introduction of the nominal beam (subscript zero) I have introduced not only the nominal enittance of the beam but also the design valies of the optical functions at the IP. It is a remarkable fact that the contributions of the bending magnets does not depend on the bean but depeneds only on the design of the line, incheling the design energy. I introrluer here the signan matrix

$$
\sigma=\left(\begin{array}{cc}
\left\langle x^{2}\right\rangle & \left\langle x x^{\prime}\right\rangle \\
\left\langle x x^{\prime}\right\rangle & \left\langle x^{\prime 2}\right\rangle
\end{array}\right)=\epsilon\left(\begin{array}{cc}
\beta & -a \\
-\alpha & \uparrow
\end{array}\right)
$$

with the condition that the determinant is equal to one giving $\gamma=\left(1+\alpha^{2}\right) / \beta$. It is simply another representation of the set of the three second-order moments of the beam distribution in one planc. the thre valables being now the emitance $\epsilon$ and the optical functions $\beta$ and (1.

Appliation to the atse of the FFTB

The FFTB line contains six main bending magnets arranged in four groups. The following table lists the individual contributions of each of these bends at the final focal point and the last line lists the resulting emittance including emittance growth. The version of the optics used for this calculation is FFTB90F and the nominal horizontal emittance is $3.010^{-10} \mathrm{~m} \cdot \mathrm{r} \cdot \mathrm{arl}$.

The most interesting result for the FFTB is the beam size increase at the IP. It is directly" obtained from $\sigma_{r}=\sqrt{\left\langle r^{2}\right\rangle}$. assinning ${ }^{\star}\langle x\rangle=0$. For the FFTB I gen $\frac{\Delta \sigma_{x}}{\sigma_{x}}=2.6 \%$.

Fonm the definition of the R.MS omittance (19) one can also obtain the emittance growth $\stackrel{y}{i}=2.7$.

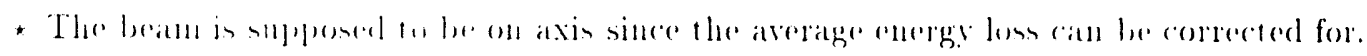




\begin{tabular}{|c|c|c|c|c|}
\hline elenent & $\left\langle. l^{2}\right\rangle$ & $\left\langle x^{\prime} 2\right\rangle$ & $\left\langle x: t^{\prime}\right\rangle$ & emittance \\
\hline $\mathrm{B}() 1$ & $1.731110^{-14}$ & $2.873510^{-13}$ & $-7.050210^{-14}$ & $1.945210^{-15}$ \\
\hline $\mathrm{B} 02$ & $2.058010^{-14}$ & $1.388710^{-13}$ & $5.106410^{-14}$ & $1.582910^{-14}$ \\
\hline $\mathrm{B}()^{2} 2$ & $4.117910^{-15}$ & $4.561610^{-13}$ & $4.091610^{-14}$ & $1.429310^{-14}$ \\
\hline $\mathrm{B}(0) 3$ & $7.998210^{-16}$ & $5.310+10^{-14}$ & $6.306310^{-15}$ & $1.644310^{-15}$ \\
\hline 1303 & $1.595+10^{-15}$ & $5.970310^{-14}$ & $-9.302910^{-15}$ & $2.950510^{-15}$ \\
\hline $\mathrm{B}() \mathrm{t}$ & $3.871910^{-15}$ & $3.780^{\prime} 110^{-14}$ & $-1.185210^{-14}$ & $2.426410^{-15}$ \\
\hline nominal & $9.010^{-i 3}$ & $1.010^{-i}$ & 0. & $3.010^{-10}$ \\
\hline total & $9.482810^{-13}$ & $1.010^{-7}$ & $6.629310^{-15}$ & $3.079410^{-10}$ \\
\hline
\end{tabular}

Table 7.1. The contribution to the enittnace growth of the different. FFTB bends. The folding of the different second order moments is described in the text.

Since we hare $\sigma_{x}=\sqrt{3 \epsilon}$, the cunitance growth alone aceounts for only about $1.3 \%$ of the spot size increase. The calculation of the enittance and optical functions using the sigma matrix clefinition above shows that there is also a change in the optical functions at the IP clue to this effert. In the case of the FFTB the relative change $\frac{\Delta \beta}{\beta}$ is about equal to the emittance growth or $2.6 \%$.

The spot size increase at the IP is :herefore due to an increase of the emittance as well as as increase of the iffunction. The emitance increase cannot be compensated for. The change in the optical functions an be compensated by retuning the line lowering the linear beta at the IP. The incrassed beta can be marle equal to the 3 -function we designed the line for in the first place. The effect has not disappeared but it would not impact the goal of oltaining a given spot size at the IP.

Lowering the , G-function at the IP cutails that it should increase at the final quadrupoles. Howerer the sime offeret that increases the 3 -function at the IP must reduce it at the final (flardrupoles since there is only a linear transport system between the two and the phase differener is $\pi / 2$. From $(21)$ it is obvions that $\left\langle x^{2}\right\rangle=36$ cammot decrease but it is possible

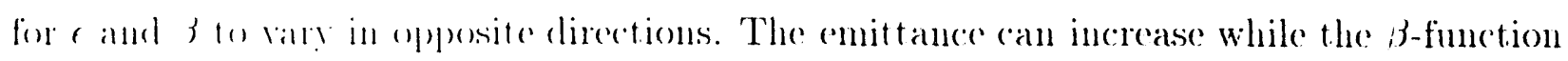
dereatsen: the net efferet still being that all second moments of the beam actually increase.

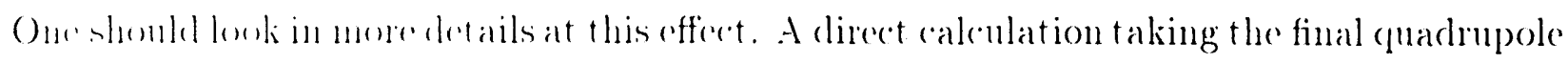
as the cout of the lime wombl I beliere andirm the above analysis. 
Note that there is also a rotation of the beam in phase spare shown by a non-zero $\left\langle x^{\prime}\right\rangle$. The beam ellipse is not upright at the nominal focal point. This however is a negligible effect, at the FF'TB. 


\section{Appendix B}

\section{Long sextupole aberration at the FFTB}

\section{INTRODUC'TION}

In a final focus system the chromaticity introduced by the very strong final lenses is cancelled at the interaction point by placing sextupoles in a clispersive region giving a chromatic kick which can be made equal and opposite to that of the final lenses.

Sextupoles are non-linear magnetic lenses giving rise to strong geometric aberrations at the focal point of a final focus system. In order to cancel some of these aberrations we place the sextupoles in pair separated by a $-I$ transformation. The thirel-oreler geometric aberrations then aneel out. However. because of the finite length of the sextupole itself, higher oreler geometric aberrations (fourth and higher) do al pear.

Liatsmolm ()ide has already estimated this effect ${ }^{[5]}$ for the FFTB but I give here a more detailed derivation of it. In addition I present another way of deriving the same result based on Lie algon)ral fordmicpues.

FIIST ()RDER TRANSP(ORT AND KICKS

Insirle a sextupole the first oreder transport is that of a drift space: the coomedinates are changing ancording to the following echations:

$$
r_{s}=r_{0}+s r_{0}^{\prime} \quad y_{s}=y_{0}+s y_{0}^{\prime}
$$

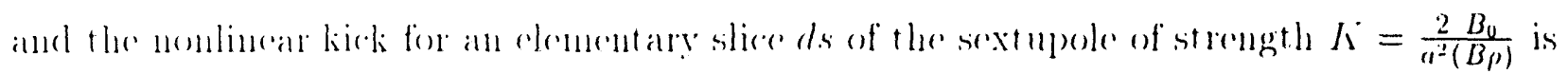
of the form:

$$
J r^{\prime}=\frac{1}{2} K\left(. r^{2}-y^{2}\right) d s . \quad \Delta y^{\prime}=-K x y d s
$$

Wi ran now take inte arement this non-linear kick in order to find the true positions $x_{s}$ and $1 \%$ alomg the sextmpole. In effect it is this slight departme from the linear trajectory

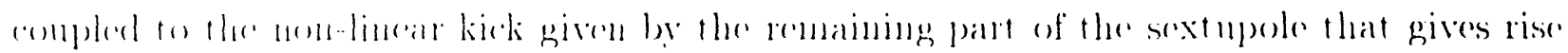

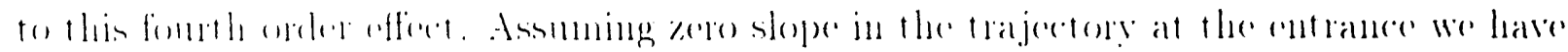




$$
\begin{aligned}
x(s) & =x_{0}+\int_{0}^{s}\left(s-s^{\prime}\right) \Delta x^{\prime}\left(s^{\prime}\right) \\
& =x_{0}+\int_{0}^{s}\left(s-s^{\prime}\right) \frac{K}{2}\left(x_{0}^{2}-y_{0}^{2}\right) d s^{\prime} \\
& =x_{0}+\frac{K}{4} s^{2}\left(x_{0}^{2}-y_{0}^{2}\right) \\
\text { and similarly } \quad y(s) & =y_{0}-\frac{K}{2} s^{2} x_{0} y_{0}
\end{aligned}
$$

Replacing $x$ and $y$ in equation (2) by their expression in equation (3) and integrating over the length of the sextupole we get the total change in angle or equivalent kick. We evaluate here only the vertical kick as this is the critical plane for flat beam final focus systems.

$$
\begin{aligned}
\Delta y^{\prime} & =\int_{0}^{l_{s}}-\Pi x(s) y(s) d s \\
& =-\Pi \int_{0}^{l_{s}}\left[x_{0}+\frac{\kappa^{2}}{4}\left(x_{0}^{2}-y_{0}^{2}\right)\right]\left[y_{0}-\frac{K s^{2}}{2} x_{0} y_{0}\right] d s \\
& =-K l_{s} x_{0} y_{0}-\frac{\Pi^{-2} l_{s}^{3}}{12} y_{0}\left(x_{0}^{2}+y_{0}^{2}\right)+\frac{\Pi^{-3} l_{s}^{5}}{40} x_{0} y_{0}\left(x_{0}^{2}-y_{0}^{2}\right)
\end{aligned}
$$

The first term in the above equation is the normal sextupolar kick (second order) that we use to correct the chromaticity ${ }^{\star}$. It is also the reason why we introduce the sextupoles in pairs so that their purely geometric parts cancel when considering the entire chromatic correction section. ${ }^{[55]}$ Indeed all geometric terms of even order, such as the third term forder four) above, do cancel across the $-I$.

The second term is that of interest to us and represents the long-sextupole, ortupole-like kick of oreler three.

C'onsidering finally the global kick given by a pair of sextupoles separated by a $-I$ transformation we get the long-sextupole non-linear kick at the end of the second sextupole

* the sextupoles are placed in a dispersive region so just change $x$ into $x+\eta \delta$ to see the chromatic term alpear. 
of the pair

$$
\Delta y^{\prime}=-\frac{K^{-2} l_{s}^{3}}{6} y_{0}\left(r_{0}^{2}+y_{0}^{2}\right)
$$

or with $k_{s}=K l_{s}$ the integrated strength of the sextupole:

$$
\Delta y^{\prime}=-\frac{k_{s}^{2} l_{s}}{6} y\left(x^{2}+y^{2}\right)
$$

\section{Lie algelra DERIVATION}

I will now use Lie algebra based t chniques to derive the same result. I hope that the reader will profit from this parallel derivation and will catch a glimpse of the ease and possibilities of this method for higher orders.

The hamiltonian of a slice ds of sextupole can be given by

$$
H d s=\frac{\pi}{3 !} d s\left(x^{3}-3 x y^{2}\right)
$$

The effect of a long sextupole is obtained by combining the hamiltonians of these elementary slices into one global haniltonian for the whole magnet according to the CampbellBalicr-Hansdorff theorem which can be expressed in our case

$$
H_{\text {whole }}=\sum_{i} H\left(s_{i}\right) d s_{i}+\frac{1}{2} \sum_{i} \sum_{j>i}\left[H\left(s_{i}\right) d s_{i}, H\left(s_{j}\right) d s_{j}\right]+\ldots
$$

The first term in the abore formula is simply the total sextupole kick we use for chromaticity correction. The second term represents the interaction of the slices of sextupole two by two and is the term we are interested in. Note that there are more terms in this expansion and although they are not significant here they are also very casy to calculate, involving only more Poisson brackets. 
Fon transforming the sums into integrals oree the length of the magnet the long sextupole term is written

$$
\begin{aligned}
H_{l s} & =\frac{1}{2} \int_{-L / 2}^{L / 2} d s \int_{s}^{L / 2} d s^{\prime}\left[H(s), H\left(s^{\prime}\right)\right] \\
& =\frac{L^{\cdot 2}}{72} \int_{-L / 2}^{L / 2} d s \int_{s}^{L / 2} d s^{\prime}\left[x(s)^{3}-3 x(s) y(s)^{2}, . . \cdot\left(s^{\prime}\right)^{3}-3 x\left(s^{\prime}\right) y\left(s^{\prime}\right)^{2}\right]
\end{aligned}
$$

Using relations like

$$
\left[x\left(s^{3} x\left(s^{\prime}\right) y\left(s^{\prime}\right)^{2}\right]=3 x(o)^{2} y\left(s^{\prime}\right)^{2}\left[x(s), x\left(s^{\prime}\right)\right]=3 x(s)^{2} y\left(s^{\prime}\right)^{2}\left(s^{\prime}-s\right)\right.
$$

the Poisson bracket can be expressed in terms of the elementary $\left[x_{i}(s), x_{j}\left(s^{\prime}\right)\right]$ which are evaluated from the linear optics in the element: e.g. $\left[x(s), x\left(s^{\prime}\right)\right]=\left[x(s), x(s)+\left(s^{\prime}-s\right) \cdot x^{\prime}(s)\right]=$ $s^{\prime}-s$.

Integrating over the lengths one needs only keep the lowest order in $\mathrm{L}$ which is equivalent to approximating $x(3)$ by its value at the magnet center $x_{c}$ before integrating. This is justified by the fact that terms of higher order in $\mathrm{L}$ involve also the angles of the trajectory at the center of the magnet. $x^{\prime}{ }_{c}$ and $y^{\prime}{ }_{c}$ which are typically two or more orders of magnitude smaller than the $x_{c}$ and $y_{c}$ due to very high beta functions at the sextupoles in final focus systems.

We finally have the following hamiltonian for the long sextupole effect:

$$
H_{l s}=\frac{K^{-2} L^{3}}{48}\left(x_{c}^{2}+y_{c}^{2}\right)^{2}
$$

In order to find the offect of this hamiltonian on the trajectory, one needs only take its Poisson bracket with the corresponding coordinate function. For example the long sextupole rortical kick is giron by

$$
\Delta y^{\prime}=\left[H_{l s \cdot} \cdot y^{\prime}\right]=\frac{\partial H_{l s}}{\partial y}=\frac{K^{-2} l_{s}^{3}}{12}\left(y_{r}^{3}+y_{r} \cdot x_{c}^{2}\right)
$$

Taking into acconnt a sextupole pair separated by a $-I$ transformation one finds the same kick we had in equation (6). 


\section{Consequence on SPOT SIZE InCREASE}

K. Oide making an estimate of this effect ( $c f$. SL.AC'-Pub 4953) argues that since the contribution of the horizontal planc is small compared to that of the vertical plane at the second pair of sextupoles, we can retain only the term in $y^{3}$ in ecpuation (6). Since the beam sizes are comparable in our case $\left(\sigma_{y} \sim 1.6 \sigma_{x}\right)$ this statement may not be obvious at first glance. I carry ont here the detailed caleulation.

The displacement at the interaction point corresponding to this kick is

$$
\Delta y^{*}=\sqrt{\beta_{y_{s}} \beta_{y}^{*}} \frac{k_{s}^{2}}{6} l_{s} y\left(x^{2}+y^{2}\right)
$$

and the spot size increase is given by

$$
\Delta \sigma_{y}^{* 2}=\left\langle\Delta y^{* 2}\right\rangle-\left\langle\Delta y^{*}\right\rangle^{2}
$$

Me assume in the following that there is no compling in the optics so that the variables $x$ and y are not comelated. The two terms of equation (14) then evaluate to the following, neglecting the change in centroid position induced by the sextupoles

$$
\begin{aligned}
\left\langle y\left(x^{2}+y^{2}\right)\right\rangle & =\left\langle y^{3}\right\rangle+\langle y\rangle\left\langle x^{2}\right\rangle=0 \\
\text { and } \quad\left\langle y^{2}\left(x^{2}+y^{2}\right)^{2}\right\rangle & =\left\langle y^{6}\right\rangle+2\left\langle y^{4}\right\rangle\left\langle x^{2}\right\rangle+\left\langle y^{2}\right\rangle\left\langle x^{4}\right\rangle
\end{aligned}
$$

Using the formula $\left\langle z^{2 n}\right\rangle=(2 n-1) ! !\left\langle z^{2}\right\rangle^{n}$ for Ganssian distributions we get

$$
\left\langle y^{2}\left(. l^{2}+y^{2}\right)^{2}\right\rangle=15 \sigma_{y}^{6}+6 \sigma_{y}^{4} \sigma_{x}^{2}+3 \sigma_{y}^{2} \sigma_{x}^{4}
$$

Finally for the spot size increase we have, with the cluantities on the right hand side evaluated at the sextupoles

$$
\Delta \sigma_{y}^{* 2}=\beta_{y} 3_{y}^{*} \frac{k_{s}^{4} l_{s}^{2}}{36}\left(15 \sigma_{y}^{6}+6 \sigma_{y}^{4} \sigma_{. x}^{2}+3 \sigma_{y}^{2} \sigma_{. x}^{4}\right)
$$

or erpuivalently

$$
\frac{\Delta \sigma_{y}^{* 2}}{\sigma_{y}^{* 2}}=\frac{j}{12} k_{s}^{4} l_{s}^{2}, 3_{y}^{4} \epsilon_{y}^{2}\left(1+\frac{2}{j} \frac{\sigma_{x}^{2}}{\sigma_{y}^{2}}+\frac{1}{5} \frac{\sigma_{x}^{4}}{\sigma_{y}^{4}}\right)
$$

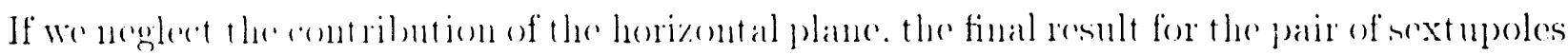


is

$$
\frac{\Delta \sigma_{y}^{* 2}}{\sigma_{y}^{* 2}}=\frac{5}{12} k_{s}^{4} l_{s}^{2} \beta_{y}^{4} \epsilon_{y}^{2}
$$

RESULTS FOR THE FFTB

In the case of the FFTB we anticipate that the largest contribution comes from the second pair of sextupoles where the beta functions are much larger than at the first pair. We consider here the two pairs of sextupoles as independent and giving rise to independent aberrations that need to be added in quarlrature to find the overall effect.

\section{$\mathrm{CCY}$}

Let us first examine the second pair of sextupoles, located in the seconc' chromatic correction section or $\mathrm{CCY}^{\star}$. The integrated strength of the sextupoles is $k_{\mathrm{s}}=21.30 \mathrm{~m}^{-2}$ with a length $l_{s}=0.25 \mathrm{~m}$ and the vertical beta function is $\beta_{y}=10135 \mathrm{~m}$ with a vertical emittance of $\epsilon_{y}=3 \cdot 10^{-11} \mathrm{~m} . \mathrm{rad}$. Finally the ratio of the horizontal to vertical spot size is $\sigma_{x} / \sigma_{y}=0.66$ and we have

$$
\begin{aligned}
\frac{\Delta \sigma_{y}^{* 2}}{\sigma_{y}^{* 2}} & =0.051 \times(1+.174+.038) \\
& =0.062
\end{aligned}
$$

which gives rise to a $\Delta \sigma_{y}^{*} / \sigma_{y}^{*}=1-\sqrt{1+.062} \approx 3 \%$ increase in the final vertical spot size.

Neglecting the contribution of the horizontal plane we have $\Delta \sigma_{y}^{*} / \sigma_{y}^{*} \approx 2.5 \%$. We see here that the approximation of neglecting the horizontal contribution is justified a posteriori.

\section{CCX}

The parameters for the first pair of sextupoles are $k_{s}=21.57 \mathrm{~m}^{-2} \cdot l_{\mathrm{s}}=0.25 \mathrm{~m} . \mathrm{j}_{\mathrm{y}}=$

* The values quoted here are those for the FFTB91D version of the FFTB optics 
$268 m$ and $\sigma_{x} / \sigma_{y}=10.27$ giving

$$
\begin{aligned}
\frac{\Delta \sigma_{y}^{* 2}}{\sigma_{y}^{* 2}} & =2.6210^{-8}(1+42+2225) \\
& =6 \cdot 10^{-5}
\end{aligned}
$$

which is negligible compared to the CCY. Here the horizontal contribution is far dominant over the vertical one as was expected.

\section{Conclusion}

We have estimated the effect of the length of the sextupoles on the final spot size at the FFTB. This aberration comes quasi exclusively from the second sextupole pair and taking into account only the vertical contribution is a good approximation. The effect is expected to increase the vertical spot size by about $3 \%$ of the linear size. It could be corrected by introducing an octupole in the line. presumably just before the final quadrupoles since it has to be in a dispersion free region in order to avoid introdncing new chromatic aberrations. Althomgh the offect is not very important at the $3 \%$ level, it is giving a higher contribution than the limit we establish to estimate the tolerances of the elements of the beam line to different errors. $[1 \cdot]$ 


\section{REFERENCES}

1. SL.AC Lincar Collider Design Handhook, 1984

2. P. Bambade (SLAC). Optical Tuning in the Ares and Final Focus Sections of the Stanford Linear Collider. SLAC'-0340, Mar 1989. Ph.D. Thesis. Also LAL-89-02 in Frenclis.

3. Final Focus Test Beam: Project Design Report. SLAC'-0376, Mar 1991.

4. FFTB Optic's Handhook. In preparation.

5. Katsumobn ()icle (SLAC). Design of optics for the Final Focus Test Beam at SLAC. SLAC'-PLB-4953. May 1989.

6. E.D. Courant and H.S. Suycler. Ann. Plụs. (X.Y.) 3, 1 (1958).

7. David C'. Carey (F.Y.LL). The optic's of Charged Particle Beams, Harwood Academic: Publishers. (1980)

8. Larl L. Brown. Frank Rotlacker. David C. Carey, F.C. Iselin (SLAC), TRANSPORT: a computer program for designing charged particle beam transport systems. SLAC0091 Rex. 2. May 19Ti.

9. Hans Grote. F.Christoph Iselin (CER.'). The Mad Program (methodical Accelerator

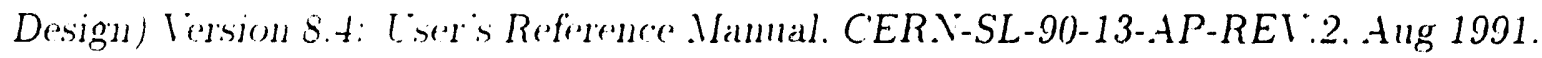

10. R. Servanclix (Saskatchewan L', and SLAC), Karl L. Brown (SLAC), Lindsay Schachinger. David Domglas (SSCL). Csers Guide to the Program Dimad, SLAC0285. Nay 1980 .

11. Darid ('. Carey (Fomilab). Third Order Transport with Marl Input. FERMILABT.1-1.j.6. Sig 1988.

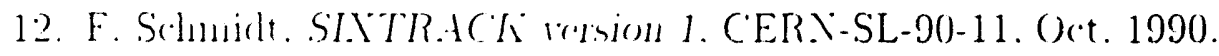

13. A.J. Dragt. L..M. Hoaly. F Niri. R.D. Rrue (Maryland L.). D.R. Donglas. E. Forest (LBL. Buteley). Marrlir 3.0) A Program for Vonlinear Analysis of Arecelerator and Bran Line Lattices. IEEE Trans. Nurel. Sir. 32 ( 1985) 2311-2313. 
14. M. Ber\% and H. Mollnik (Ciessen U.). The program Hamilton for the analytic solution of the equations of motion through fifth order. Nucl. Instr. Meth. A258 (1985) 364-373.

15. M. Ber\% (Midigan State L.). Lsers' guide to C'OSY-Infinity: The code can be obtained directly from its author.

16. J. Irwin. S. Peggs (SSCL), X.MAP: A Differential Algebra Tool Generating Accelerator Maps. SSC-N-6()4. Mar 1989.

17. T. Sen. Y' Yan (SSCL). J. Irwin (SLAC), LIEMAP: A Program for Extrating a one Turn Single Exponent Lie Ginerator Map). SSCL-443, May 1991.

18. A.J. Dragt (Maryland U.). Lectures on Nonlinear Orbit Dynamics. In Batavia 1981, Proceedings. Physics Of High Energy Particle Accolerators, AIP Conf. Proc. 87, $1+7-313,1981$.

19. A.J. Dragt. J.M. Finn (.Maryland L.). Lie Series and Inrariant Functions or Analytic Simplectic . Mapss. J. Mathematical Phỵs.17 (1970) 2215-2227, 1970

20. John Irwin (SLAC). The Application of Lie Algabra Techniques to Beam Transport Designn. SLAC'-PLB-5315. Ang 1990. Submitted to Niucl. Instrum. Methods A.

21. Karl L. Brown (SLAC), R. Servanckx (Saskatchewan U.). A Magnifying Magnetic Optical Achromat. Nucl. Instr. Math. 203 (1982) 73.

22. Larl L. Brown (SLAC). A Serond Order Magnetic Optical Achromat. SLAC'-PUB2.257. Fol, 1979. 32pre. Submitted to Nincl. Instr. Mothorls

23. John Irwin. Cihislain Roy (SLAC') A comparison of two final forms sistems for lincar

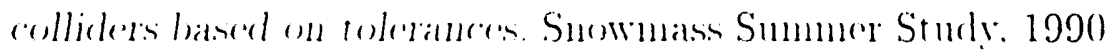

24. Tor Rambenherinter. private (omumumication.

25. John Secman. private communication.

26. Jim Spentere private communiagtion.

27. R. Brinkmann (D)ESY). Optimization of a final forms stostem for large momentum handwidth. DEST - I-O()-14. Xo 1990.

28. K. ()irle. private (o)mmmuliantion.

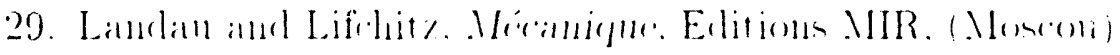


30. R.D. Ruth. Nonlinear Drmanics Aspects of Particle Acerlemators. Lecture Votes in Physics, 247, Springer. Berlin, 1986.

31. this solution was easily obtained, along with other results in this thesis, using the program Mathematica,a system for doing mathematics with computers. It is also developped in the next reference.

32. W.E. Gabella, PhD. Thesis (1991),

33. Stephen Wolfram, Mathematica, a system for doing mathematics by computer.

34. H. Wollnik and M. Berz (Giessen U.), Relations between elements of transfer matrices due to the condition of symplecticity, Nucl. Instrum. Meth. A238 (1985) 127-140.

35. Karl L. Brown (SLAC), A Conceptual Design of Final Focus Systems for Linear Colliders. SLAC'-PLB-4159, Jun 1987.

36. J. Buon, F. Couchot, J. Jeanjean, F. Lc Diberder, V. Lepeltier, H. Nguyen Ngoc, J. Perez-y-Jorba, P. Puzo (Orsay, LAL), P. Chen (SLAC), Proposal of a Beam Size Monitor for the Final Focus Test Beam. SLAC-AAS-NOTE-52, Sep 1990.

37. J. Buon, F. Couchot, J. Jeanjean, F. Le Diberder, V. Lepeltier, H. Nguyen Ngoc, J. Perez-y-Jorba. P. Puzo (Orsay, LAL), P. Chen (SLAC), Beam Size Monitor for the Final For'us Test Beam. Nucl. Instrum. Meth. A306 (1991) 93-111.

38. J. Buon. F. Conchot. J. Jeanjean, F. Lediberder, V. Lepeltier, H. Nguyen Ngoc, P. Puzo (Orsay, LAL). P. Chen (SLAC'), Status Report on the Beam Size Monitor for FFTB. Print-90-0418. Jul 1990.

39. John Irwin, D.L. Burke (SLAC), Linear Collider IR and Final Focus Introduction. SLAC-PUB-5652, Sep 1991. Presented at DPF Summer Study on High Energy Physics: Research Directions for the Decade. Snowmass, CO, Jun 25 - Jul 13, 1990.

40. D.L. Burke et al., Linear Colliders. SLAC-PUB-5597, .Jul 1991.

41. John Irwin (SLAC). Towarels the Vext Linear Collider. SLAC-PLB-j651, Sep 1991.

42. G. Roy, F. Bulos, D.L. Burke, Richard H. Helm, John Irwin, N. Yamamoto (SLAC), Review of Tolerances at the Final Focus Test Beam, SLAC-PUB-5485, May 1991. 
43. John Irwin. Larl L. Brown, F. Bulos, D.L. Burke, Richard H. Holm, G. Roy, R.D. Ruth. N. Yamanoto (SLAC). Katsunolm Oide (KEK, Tsukuba). The Optics of the Final Foc'us Test Beam. SLAC'-PLB-j539. May 1991.

44. Richard H. Helm (SLAC), First and second order beam optics of a curved, inclined mannetic field boundary in the impulse approximation. SLAC-0024, Nov 1963.

45. F. Bulos, D.L. Burke, Richard H. Helm, John Irwin, A. Odian, G. Roy, R.D. Ruth, N. Yamamoto (SLAC), Beam Based Alignment and Tuning Procedures for $e^{+} e^{-}$Collider Final Foc'..; Systems. SLAC-PUB-5488, May 1991.

46. Joln Irwin (SLAC), Construction of High Order Maps for Large Proton Accelerators. SLAC'-PUB-5486, May 1991.

47. Lia Merminga, John Irwin, Richard H. Helm, R.D. Ruth (SLAC), Optimizing a Nonlinear Collimation System for Future Linear Colliders. SLAC-PUB-5507, May' 1991.

48. Lia Merminga, Joln Irwin, Richard H. Helm, R.D. Ruth (SLAC), Collimation Systems in the Next Linear Collider. SLAC-PUB-5436, Feb 1991.

49. Chistain Roy (Orsay, LAL). An Effect of the Fringing Field in Sector Bending Magnets: the coupling of the Transverse Planes in the Solutions of the Equation of Motion at Second Order. LAL-88-53, Nov 1988.

50. Karl L. Brown (SLAC') A First and Second Order Matrix Theory for the Design of Beam Transpont Systems and Charged Particle Spectrometers. SLAC'-0075-rev-4, Jun 1982.

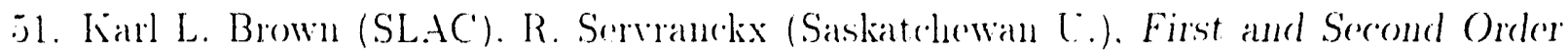
Charged Particle Optics. SLAC'-PUB-3381, Jul 1984.

52. M. Sands (SLAC), Emittance Growth from Radiation Fluctuations. SLAC/AP-()47, Der 1985.

53. A Final Focus System for Flat Beam Lincar Colliders. By Katsumobu Oide (SLAC), SLAC'-PLB-4660). Jum 1988. Nucl.Instr..Meth. A270:427,1989.

j4. K. ()ide (SLAC), Sinchrotron Radiation Limit on the Focusing of Elertron Barms. Phiss. Rorv. Lott. 61 (1088) 1713-171j. 


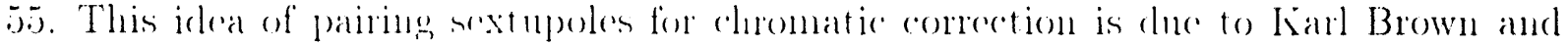
Roger Serranclex and was first introduced for the second oreler achromat.

56. these tolerances were calculated by John Irwin nsing a Mathematica program to (alculate the size and effects of bumps across the lattice. 

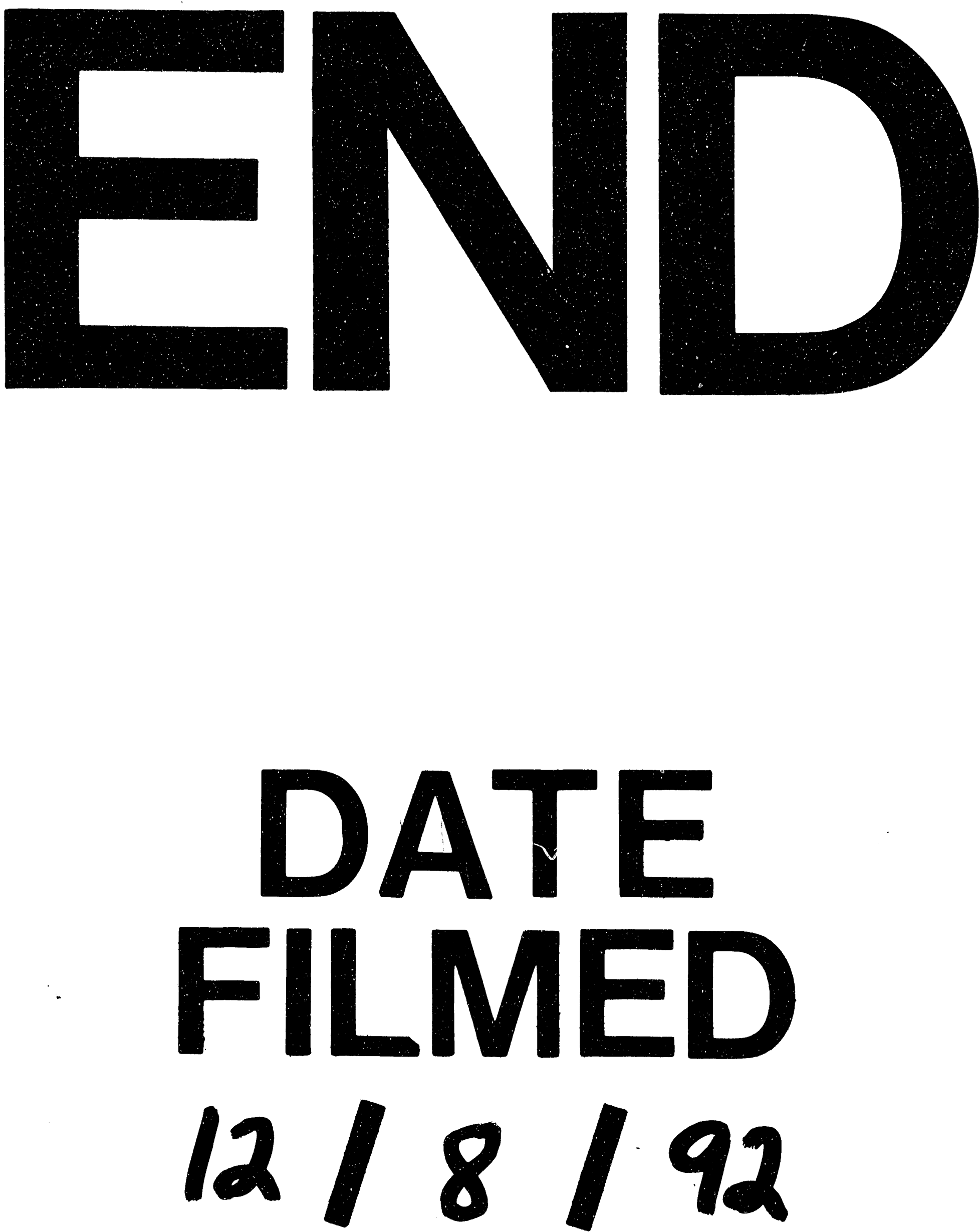
